Aus der Abteilung Neurochirurgie

( Prof. Dr. med. V. Rohde)

im Zentrum Neurologische Medizin

der Medizinischen Fakultät der Universität Göttingen

\title{
Glioblastomtherapie von 1998 bis 2004 in der Universitätsklinik Göttingen
}

Eine retrospektive Analyse zeitgemäßer Behandlungsstrategien

INAUGURAL - DISSERTATION

zur Erlangung des Doktorgrades

der Medizinischen Fakultät

der Georg-August-Universität zu Göttingen

vorgelegt von

Yvonne Echegoyen Hornfeldt

aus Laudenbach

Göttingen 2010 
Dekan: Prof. Dr. med. C. Frömmel

I. Berichterstatter: Prof. Dr. med. A. Giese

II. Berichterstatter/in:

III. Berichterstatter/in:

Tag der mündlichen Prüfung: 


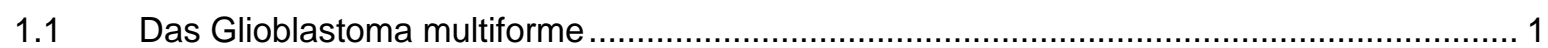

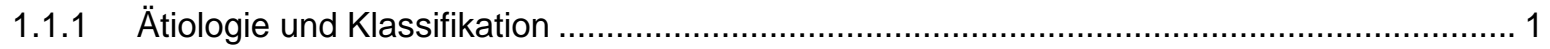

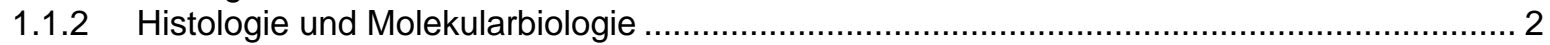

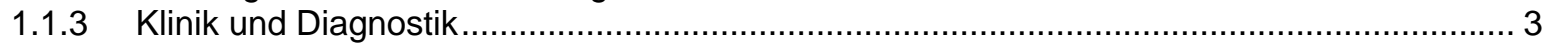

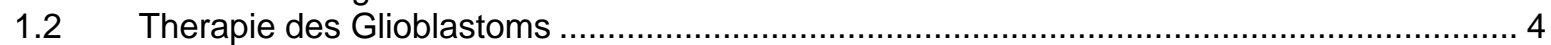

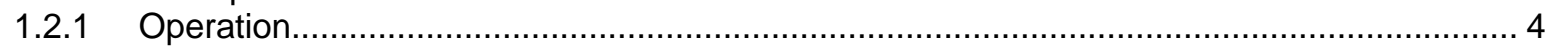

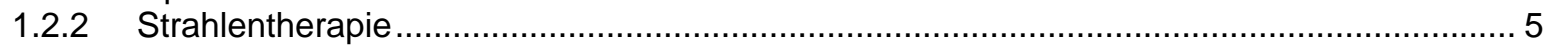

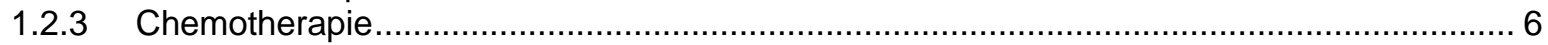

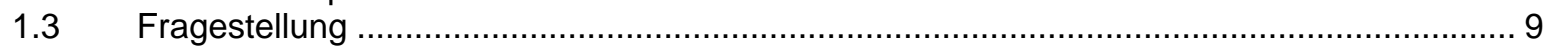

2 PATIENTEN UND METHODEN ......................................................................................................... 10

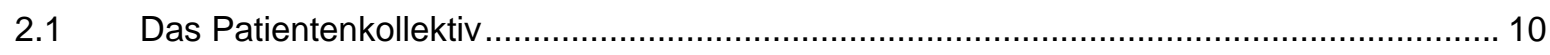

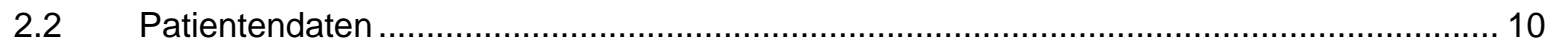

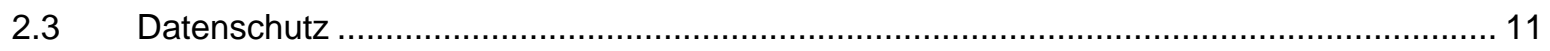

2.4 Datenerfassung über eine formularbasierte Datenbank ............................................ 11

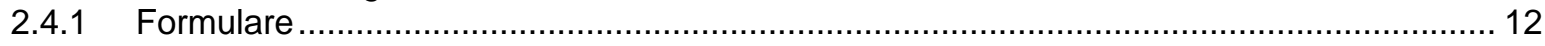

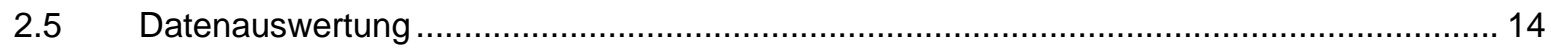

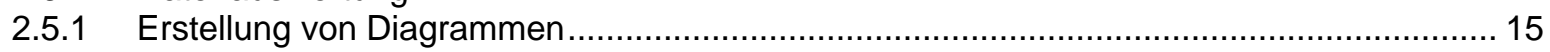

2.5.2 Erstellung von Survivalanalysen und Progressanalysen ............................................ 15

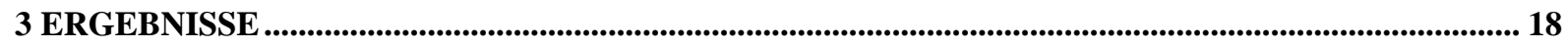

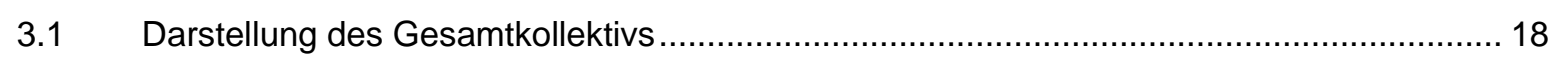

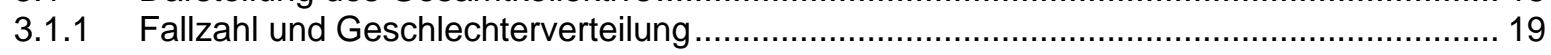

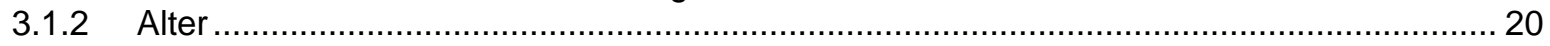

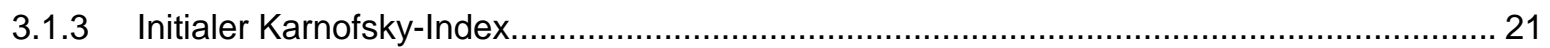

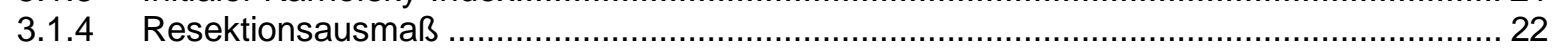

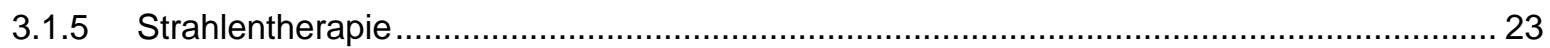

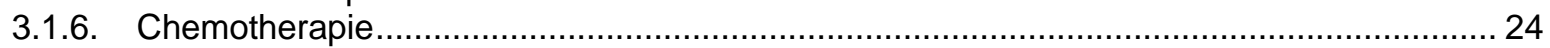

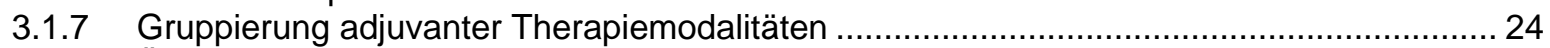

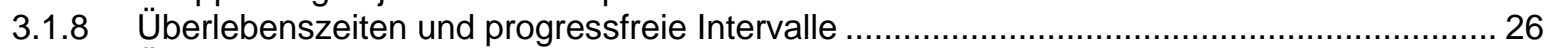

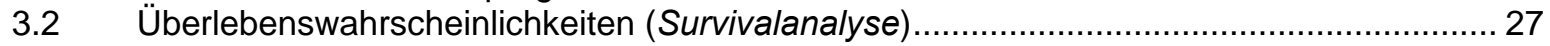

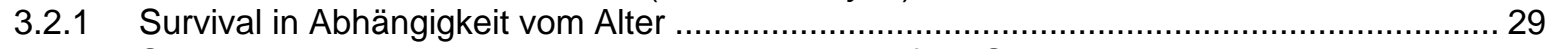

3.2.2 Survival in Abhängigkeit vom präoperativen Karnofsky-Status ....................................... 31

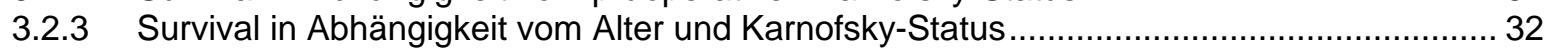

3.2.4 Survival in Abhängigkeit vom Resektionsausmaß ....................................................... 34

3.2.5 Survival in Abhängigkeit von der postoperativen, adjuvanten Therapie ............................ 35

3.2.6 Survival in Abhängigkeit von Resektionsausmaß kombiniert mit adjuvanter Therapie ....... 39

3.2.7 Survival in Abhängigkeit vom Zytostatikum .................................................................. 41

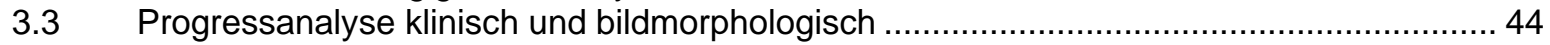

3.3.1 Progressionsanalyse in Abhängigkeit von Alter und Karnofsky-Index .............................. 44

3.3.2 Progressionsanalyse in Abhängigkeit vom Resektionsausmaß ..................................... 46

3.3.3 Progressionsanalyse in Abhängigkeit der postoperativen, adjuvanten Therapie .................. 48

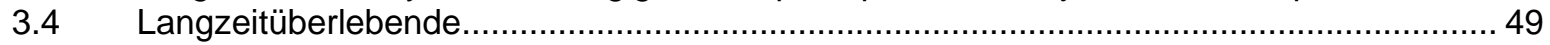

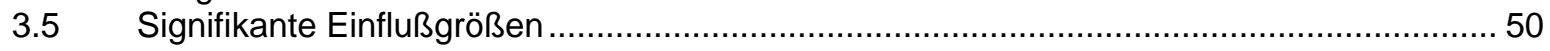

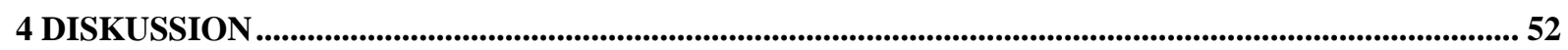

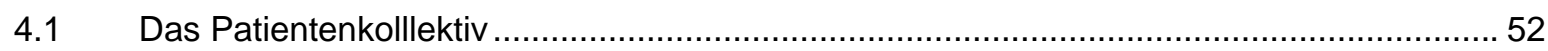

$4.2 \quad$ Alter und Karnofsky-Index zum Zeitpunkt der Erstdiagnose.......................................... 52

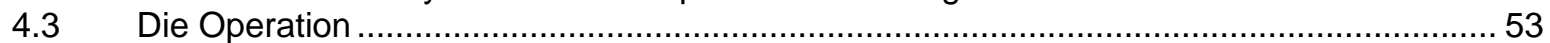

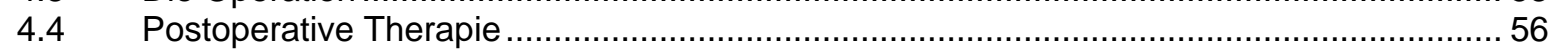

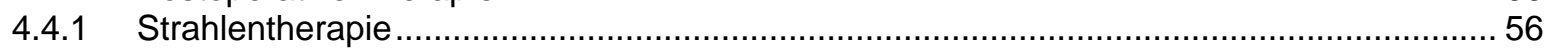

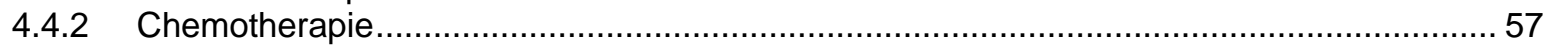

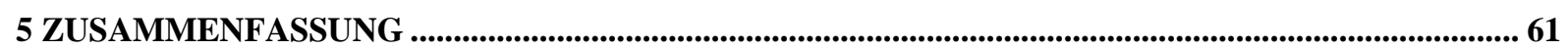

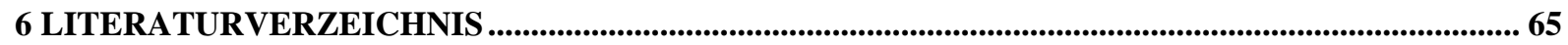




\section{Einleitung}

\subsection{Das Glioblastoma multiforme}

\subsection{1 Ätiologie und Klassifikation}

Die Gliome gehören zu der Gruppe neuroepithelialer Tumoren. Die Inzidenz innerhalb der Normalbevölkerung liegt bei fünf bis zehn Erkrankungen pro 100000 Einwohner (Legler et al. 1999). Die malignen Gliome sind mit etwa 30\% die häufigsten und zugleich aggressivsten Hirntumoren glialen Ursprungs. Der Gipfel der Erkrankungshäufigkeit liegt zwischen dem 5. und 6. Lebensjahrzehnt.

Die Einteilung der Gliome erfolgt anhand der WHO-Klassifikation. Die erste WHOKlassifikation der Tumoren des zentralen Nervensystems geht auf das Jahr 1979 zurück. Dieser folgte eine Überarbeitung von 1993, in der bereits immunhistologische Kriterien berücksichtigt wurden (Kleihues et al. 1993). Insbesondere neue Erkenntnisse im Bereich der Molekulargenetik, die zum Beispiel zur Identifizierung von 1p und 19q Alterationen als Marker für ein gewisses Therapieansprechen und biologisches Verhalten der Oligodendrogliome führten, haben 1999 zu einer neuen Version der WHO-Klassifikation geführt (Kleihues et al. 2002).

Hier unterscheidet man drei Hauptgruppen der Gliome. Zum einen die Astrozytome, die Oligodendrogliome und die Oligoastrozytome. Während die sekundären Glioblastome durch Malignisierung besser differenzierter Astrozytome entstehen, wachsen die primären Glioblastome de novo (Kleihues und Ohgaki 1999). Bisher geben retrospektive Studien keinen Anhalt dafür, dass maligne Gliome, die aus einem Gliom zweiten Grades hervorgegangen sind, eine bessere Prognose als primär maligne Gliome haben (Dropcho und Soong 1996). Die Einteilung in die Malignitätsgrade der Tumoren hängt im Wesentlichen von der Anzahl der Kernatypien, der Mitoserate, der mikrovaskulären Proliferation und dem Auftreten von Nekrosen ab. So werden die einzelnen Hauptgruppen nochmals aufgrund der zunehmenden Anaplasie unterteilt. Bei den astrozytären Gliomen unterscheidet man das pilozystische Astrozytom (WHO Grad I), das diffuse Astrozytom (WHO Grad II), das anaplastische Astrozytom (WHO Grad III) sowie die bösartigste Form, das Glioblastoma multiforme (WHO Grad IV) (He et al. 2001). Gliosarkome, die als Merkmal mesenchymale und neuroektodermale Elemente aufweisen, verhalten sich wie Glioblastome (Galanis et al. 1998). 


\subsubsection{Histologie und Molekularbiologie}

Glioblastome sind charakterisiert durch Zellreichtum und Anaplasie mit hoher mitotischer Aktivität sowie dem Vorkommen von Nekrosen und mikrovaskulärer Proliferation (Kleihues et al. 2002). Verschiedene Studien haben Hinweise dafür ergeben, dass bestimmte histologische Charakteristika Einfluss auf den klinischen Verlauf der Erkrankung haben. So ist beispielsweise das Auftreten von Nekrosen im histopathologischen Präparat als schlechter prädiktiver Faktor beschrieben (Barker et al. 1996). Oligodendrogliale Komponenten hingegen gelten eher als positiver prädiktiver Faktor und sind in einigen Untersuchungen häufig mit einem vergleichsweise längeren Überleben vergesellschaftet (Hilton et al. 2004). Des Weiteren wurden mögliche Zusammenhänge zwischen histopathologischen Eigenschaften und genetischen Alterationen untersucht. Hierbei zeigte sich insbesondere eine Amplifikation von EGFR (epidermal growth factor) in besonders kleinzelligen Strukturen, wie sie im Glioblastom typisch sind (Burger et al. 2001).

Wie bei anderen bösartigen Tumoren findet sich auch bei Glioblastomen eine veränderte Expression von Onkogenen und Tumorsuppressorgenen.

Eine genetische Alteration mit signifikanter Häufung in primären Glioblastomen, verglichen mit sekundären, aus niedergradigeren Tumoren entstandenen Gliomen, betrifft die epidermale Wachstumsfaktor-Genamplifikation. Eine Überexpression von EGFR wird in $40 \%$ bis $50 \%$ der molekularbiologischen Untersuchungen nachgewiesen (Watanabe et al. 1996). Die TP-53-Mutation hingegen findet sich signifikant gehäuft in niedriggradigen Gliomen und sekundären Glioblastomen (Gil-Benso et al. 2007). Mit dem Nachweis des Verlustes der heterozygoten Form vom Chromosom 10q (LOH 10q) konnte eine ausgesprochen häufige Genveränderung primärer Glioblastome beobachtet werden (Zhou et al. 1999). Diese Alteration ist als einzige mit einem signifikant kürzeren Überleben assoziiert (Ichimura et al. 2004).

Die makroskopische Schnittfläche des Tumors zeigt ein Nebeneinander von gelblichen Nekrosen, zystischen Zerfallshöhlen, Hämorrhagien und zellreichen Anteilen. Während sich das Tumorareal makroskopisch gut gegen das umgebende Ödem abgrenzen lässt, zeigt sich mikroskopisch eine variable Zone infiltrierten Gehirns rund um das Tumorgebiet. Invasive Gliomzellen haben eine ausgeprägte Affinität zu myelinisierten Faserzügen. Entsprechend ist die Ausbreitung der Gliome entlang der weißen Substanz durch die Balkenstrahlung oder entlang der Wände des Ventrikelsytems zu beobachten (Giese und Westphal 1996). 


\subsubsection{Klinik und Diagnostik}

Die häufigsten klinischen Manifestationen betroffener Patienten sind der zerebrale Krampfanfall, wobei dieser sowohl primär fokal als auch sekundär generalisiert oder generalisiert auftreten kann. Kopfschmerzen, Schwindel, Übelkeit und Bewusstseinsstörung können Ausdruck von steigendem intrazerebralem Druck sein. Außerdem werden fokale neurologische Defizite, wie Aphasie oder Hemiparese beobachtet. Fremdanamnestisch werden häufig Persönlichkeitsveränderungen beschrieben. Von den 2000-3000 jährlich diagnostizierten Patienten in Deutschland mit Glioblastom verstirbt etwa die Hälfte innerhalb eines Jahres (Behin et al. 2003). Da keine spezielllen Tumormarker für Liquor oder Serum zur Verfügung stehen, gilt das MRT derzeit als Wegweiser für die Diagnosefindung. Ergibt sich anhand von Klinik und zusätzlich durchgeführter Computertomographie der Verdacht auf einen Tumor, wird eine kontrastmittelgestützte Kernspinuntersuchung in T1- und T2-Wichtung durchgeführt. Das typische MR-tomographische Erscheinungsbild der Glioblastome zeigt einen Gadolinium-anreichernden girlandenartigen Tumoranteil mit zentraler Nekrose umgeben von einem mehr oder weniger ausgeprägten perifokalen Ödem. Gelegentlich finden sich in den T2- gewichteten Sequenzen Einblutungen in den Tumor und Hypervakularisation in den Perfusions- und Diffusionsuntersuchungen. In den T2- gewichteten Sequenzen stellen sich die zellreichen und soliden Anteile des Tumors hypointens dar, wohingegen sich die zystisch- nekrotischen und die für die Gliome charakteristischen eingebluteten Areale hyperintens abbilden. Aufgrund der ausgeprägten Neovaskularisation kommt es in T2- gewichteten Aufnahmen auch häufig zu einer flussbedingeten Auslöschung des Signals. Weitere bildmorphologische Charakteristika des Glioblastoms sind die raumfordernde Wirkung des Tumors und das perifokale Ödem. Eine sichere Abgrenzung zwischen infiltrativem Tumorwachstum und Ödem ist aufgrund der Bildgebung nicht möglich (Weller et al. 2006). Obwohl die Glioblastome ein aggressives Wachstum und raschen Progress zeigen, metastasieren diese Tumoren sehr selten. Mögliche Ausbreitungswege sind über die Faserbahnen, perivaskulär, subependymal und über den Liquorweg beschrieben. Die Ausbreitung ist in den meisten Fällen für die neurologische Verschlechterung der Patienten verantwortlich. In nur $10 \%$ bis $20 \%$ der Fälle kann eine leptomeningeale Aussaat als Ursache einer klinischen Verschlechterung festgestellt werden (Behin et al. 2003). Günstige prognostische Faktoren sind geringes Alter und ein guter klinischer Ausgangszustand bei Diagnosestellung, frontale Lokalisation und makroskopische Kom- 
plettresektion. Ausgeprägte Nekroseareale und perifokale Ödemkomponenten im MRT gelten als negative Prädiktoren für die Überlebenszeit (Lacroix et al. 2001).

\subsection{Therapie des Glioblastoms}

Glioblastome gelten weiterhin als unheilbar und die medizinische Behandlung dient vor allem der Palliation der neurologischen Defizite und der Verlängerung der Überlebenszeit mit bestmöglicher Erhaltung der Lebensqualität. Patienten die mit Hirndrucksymptomatik im Krankenhaus vorstellig werden, erhalten meist eine initiale Dexamethason-Behandlung zur Therapie des vasogenen Ödems. Die neurologischen Symptome des Patienten bessern sich durch den Rückgang der Raumforderung in den meisten Fällen schon innerhalb von Stunden oder wenigen Tagen. Trotz des positiven Effekts sollte die Kortisontherapie immer auf die minimale effiziente Dosis beschränkt bleiben. Zum einen aufgrund des bekannten Nebenwirkungsspektrums, zum anderen, weil Interaktionen mit Chemotherapeutika nicht auszuschließen sind (Weller et al. 1997).

\subsubsection{Operation}

Die Operation des Glioblastoms verfolgt neben der Diagnosesicherung die weitestgehende Resektion solider Tumoranteile, um durch Reduktion der Tumorlast bessere Vorraussetzung für die folgende Radio- und Chemotherapie zu schaffen. Insbesondere bei eloquenter Lage des Tumors, wie zum Beispiel im Bereich der Sprachzentren oder der motorischen Zentralregion, steht bei chirurgisch nichtkurabler Erkrankung der Erhalt der neurologischen Funktionen im Vordergrund. Um den Anspruch einer radikalen Operation mit möglichst wenig funktionellen Einbußen für den Patienten zu vereinbaren, sind heute modernes intraoperatives Neuromonitoring und intraopertive funktionelle Diagnostik auch am wachen Patienten im Einsatz. Ferner werden zur Kontrolle des Resektionsausmaßes in einigen Zentren intraoperative Kernspintomographien und standardmäßig die Neuronavigation eingesetzt (Kostron und Roessler 2006).

In bisherigen Studien konnte eine Korrelation zwischen einem aggressiven chirurgischen Vorgehen und einer Verlängerung der medianen Gesamtüberlebens-zeit oder des rezidivfreien Intervalls nachgewiesen werden (Nitta und Sato 1995). Des Weiteren belegen Studien einen Vorteil der subtotalen Resektion gegenüber der Biopsie, bezogen auf die Gesamüberlebenszeit (Jeremic et al. 1994). Kann aufgrund der Lokalisation und Ausdehnung des Tumors eine Resektion nicht erfolgen oder befindet 
sich der Patient in einem derart reduzierten Allgemeinzustand, dass eine Tumorresektion nicht sinnvoll erscheint, sollte eine stereotaktische Biopsie durchgeführt werden, um die histologische Diagnose zu sichern und ein weiteres chemotherapeutisches und/ oder radiotherapeutisches Vorgehen zu ermöglichen. Als operativ schwer behandelbar gelten bihemisphärische Tumore, die sog. „Schmetterlingsgliome“, Hirnstammgliome sowie multifokale Gliome.

Eine eindeutige Unterscheidung von Tumorgewebe und Hirngewebe ist intraoperativ auch unter mikrochirurgischen Gesichtspunkten schwierig, da die Infiltrationszone ödematös verändert ist und kaum von der gefäßreichen Wachstumszone unterschieden werden kann. Präoperativ kann die Gabe von 5-Aminolävulinsäure (5-ALA) erfolgen. Diese wird durch den Tumor zu Protoporphyrin IX verstoffwechselt und macht unter Fluoreszenzlicht solides Tumorgewebe sichtbar (Stummer et al. 2003). Tumorzellinfiltrationen können bislang weder im MRT noch im PET sichtbar gemacht werden.Tumorrezidive treten in über 90\% der Fälle innerhalb eines $2 \mathrm{~cm}$ breiten Resektionrandsaumes auf. In keinem Fall ist die chirurgische Therapie als eine kurative Behandlung anzusehen (Hofer und Merlob 2002).

\subsubsection{Strahlentherapie}

Ziel der Strahlenbehandlung ist es, den programmierten Zelltod (Apoptose) der entarteten Tumorzellen zu induzieren. Die Strahlentherapie wird heutzutage mit Hilfe von Schnittbilddatensätzen dreidimensional geplant. Je präziser das Zielvolumen definiert wird, umso exakter kann die Strahlentherapie durchgeführt werden und umso geringer sind die zu erwartenden Nebenwirkungen der Therapie.

Seit mindestens 25 Jahren gehört die Strahlentherapie zur Standardtherapie des Glioblastoms. Gegenüber der alleinigen Operation weist die adjuvante Strahlentherapie einen eindeutigen Vorteil bezüglich der erzielten Überlebenszeit auf. Beim Glioblastoma multiforme wird das mediane Überleben etwa auf 35 Wochen verdoppelt (Salazar et al. 1979, Walker et al. 1979). Des Weiteren ergaben randomisierte Studien, dass die Applikation von einer Gesamtdosis von $60 \mathrm{~Gy}$, fraktioniert in 30 Einzelanwendungen gegenüber einer Gesamtdosis von 40 Gy in 20 Sitzungen ebenfalls eine signifikante Verbesserung der Überlebenszeit erbrachte. Eine Dosis von $60 \mathrm{~Gy}$ stellt auch die Toleranzgrenze des mitbestrahlten gesunden Hirngewebes einschließlich des Hirnstammes dar. Bei einer Ganzhirnbestrahlung von 60 Gy treten nach Ablauf eines Jahres Hirnfunktionseinschränkungen bei fünf Prozent der bestrahlten $\mathrm{Pa}$ tienten auf. Die Ganzhirnbestrahlung weist keinen Vorteil bezüglich der Überlebens- 
zeit auf (Emami et al. 1991, Laperriere et al. 2002). Des Weiteren scheint die Ganzhirnbestrahlung aufgrund der sehr hohen lokalen Rezidivwahrscheinlichkeit des Glioblastoms entbehrlich, so dass heute die Bestrahlung des Tumorbettes und des umgebenden Randsaumes in einer Breite von $2 \mathrm{~cm}$ angestrebt wird (Hess et al. 1994).

\subsubsection{Chemotherapie}

Seit ungefähr 30 Jahren werden verschiedene chemotherapeutische Substanzen einzeln, in Kombination oder auch zusätzlich zu einer Strahlenbehandlung in randomisierten Studien und in Metaanalysen untersucht. Seit der Veröffentlichung der europäisch-skandinavischen Studie zur konkomitanten und adjuvanten Chemotherapie hat sich der Stellenwert von Temozolomid in der Primärtherapie von Glioblastomen verändert. Temozolomid ist ein Alkylanz, welches nach oraler Einnahme spontan in seine aktiven Metaboliten zerfällt und die Blut-Hirn-Schranke überwinden kann. Es zeigt sich, dass die zeitgleiche Chemotherapie und Strahlentherapie (konkomitante Radiochemotherapie) im Vergleich zur alleinigen Strahlentherapie die Überlebenszeit bei einem neu diagnostizierten Glioblastom signifikant verlängert. In Zahlen ausgedrückt wird das mediane Überleben um 2,5 Monate verlängert und das relative Sterberisiko um 37\% verringert (Stupp et al. 2005). Ungefähr 45\% aller Glioblastome können aufgrund einer Promotermethylierung des MGMT-Genes (Methyl-GuaninMethyl-Transferase) das korrespondierende DNA-Reperaturenzym nicht exprimieren, so dass Temozolomid-induzierte DNA-Schäden nur unzureichend repariert werden können. Bei Patienten, deren Tumor einen methylierten MGMT-Promoter enthält, wird durch die konkomitante Therapie eine Verlängerung der medianen Überlebenszeit auf 21,7 Monate erzielt. Wohingegen beim selben Patientenkollektiv, ohne verfügbaren methylierten MGMT-Promotor bei gleicher adjuvanter Therapie, die mediane Überlebenszeit bei 15,3 Monaten liegt (Hegi et al. 2005). Bei der konkomitanten Therapie werden während des gesamten Bestrahlungszeitraumes täglich $75 \mathrm{mg} / \mathrm{m}^{2}$ Temozolomid verabreicht. Danach folgt die übliche adjuvante Therapie, hierbei werden 150 bis $200 \mathrm{mg} / \mathrm{m}^{2}$ Temozolomid in einem Zyklus von 28 Tagen an den Tagen 1 bis 5 eingenommen (Stupp et al. 2005). Der Vorteil der konkomitanten Behandlung liegt im Erreichen einer höheren Gesamtdosis, ohne hierbei die Toxizität zu erhöhen (Brock et al. 1998). Gerade im Rahmen der palliativen Behandlung sollten die Nebenwirkungen eines Chemotherapeutikums gut beherrschbar sein. Übelkeit, die unter der Behandlung mit Temozolamid auftritt, lässt sich mit den üblichen Antiemetika gut behandeln, Myelosuppression führt in bis zu 5\% der behandelten Fälle zum Ab- 
bruch der Therapie (Stupp et al. 2005). Als Spätfolge von Alkylanzien wird über gehäuftes Auftreten von myelodysplastischen Syndromen sowie sekundären Leukämien berichtet (Armitage et al. 2003). Vor der Veröffentlichung der eurpäischskandinavischen Studie zur Wirksamkeit der konkomitanten Temozolomidtherapie fehlte die Evidenz, primär allen Patienten im Anschluss einer Radiotherapie eine Chemotherapie anzubieten. Aufgrund der Ergebnisse der bis zu diesem Zeitpunkt größten, prospektiven randomisierten Studie mit 600 Patienten war es gerechtfertigt, eine alleinige Radiotherapie durchzuführen (GMT Group 2002). Vor Bekanntwerden der guten Datenlage zur Wirksamkeit von Temozolomid war die Kombinationstherapie mit PCV (Procarbazin, CCNU und Vincristin) und die Therapie mit systemischen BCNU (Carmustin) und ACNU (Nimustin) ein häufig eingesetztes Therapieregime bei Gliomen mit WHO Grad III und wohl auch bei Glioblastomen. Die Oligodendrogliome wurden als besonders chemosensitive Tumoren erkannt. Es konnte ein Zusammenhang zwischen dem Verlust genetischer Information auf den Chromosomen $1 p$ und 19q und dem guten Ansprechen auf die Chemotherapie gezeigt werden (Cairncross et al. 1998). Bereits Mitte der 80er Jahre wurde die Kombinationstherapie aus Procarbazin, CCNU (Lomustin) und Vincristin bei malignen Gliomen eingesetzt. Die Zyklusdauer der PCV-Therapie beträgt 6 Wochen. Das Therapieschema sieht am ersten Tag $110 \mathrm{mg} / \mathrm{m}^{2} \mathrm{CCNU}$ oral vor, am Tag 8 bis 21 Procarbazin $60 \mathrm{mg} / \mathrm{m}^{2}$ oral und am Tag 8 und 29 je $1,4 \mathrm{mg} / \mathrm{m}^{2}$ Vincristin intravenös. Procarbazin ist wie Temodal eine alkylierende Substanz, die aufgrund ihrer Lipophilie die Blut-Hirmschranke gut penetriert und ebenfalls oral verabreicht werden kann. Wohingegen Vincristin eine ausgesprochen hydrophile Substanz ist und parenteral verabreicht werden muss. Auch die Nitroseharnstoffe CCNU, BCNU und ACNU wirken alkylierend an verschiedenen Lokalisationen der DNA (Guanosin, Adenin und Cytosin), was zu Einzel- und Doppelstrangbrüchen führt. Es handelt sich hierbei um lipophile Chemotherapeutika. Während BCNU und ACNU parenteral verabreicht werden, kann CCNU oral gegeben werden. Die wichtigsten Nebenwirkungen sind zusätzlich zur Myelosuppression Allergien, Lungenfibrosen bei BCNU sowie Polyneuropathien bei Vincristin (Weller et al. 2003). Eine randomisierte Studie zeigte 1990, dass bei Oligoastrozytomen WHO Grad III und auch bei anderen malignen Gliomen durch die adjuvante PCV-Therapie, verglichen mit der alleinigen BCNU-Therapie, sowohl eine Verbesserung der Überlebenszeit als auch des progressionsfreien Intervalls erzielt werden kann. Während die Ergebnisse in Bezug auf die histologische Gruppe der anaplastischen Astrozytome WHO Grad III statistisch signifikant waren, zeigten sich bei der Gruppe der anderen 
malignen Gliome keine statistisch signifikanten Verbesserungen von Überlebenszeit und progressionsfreiem Intervall (Levin et al. 1990). Eine Metaanalyse älterer Studien weist nach, dass die Kombinationstherapie mit PCV einer Monotherapie mit BCNU insgesamt nicht überlegen ist (Prados et al. 1999).

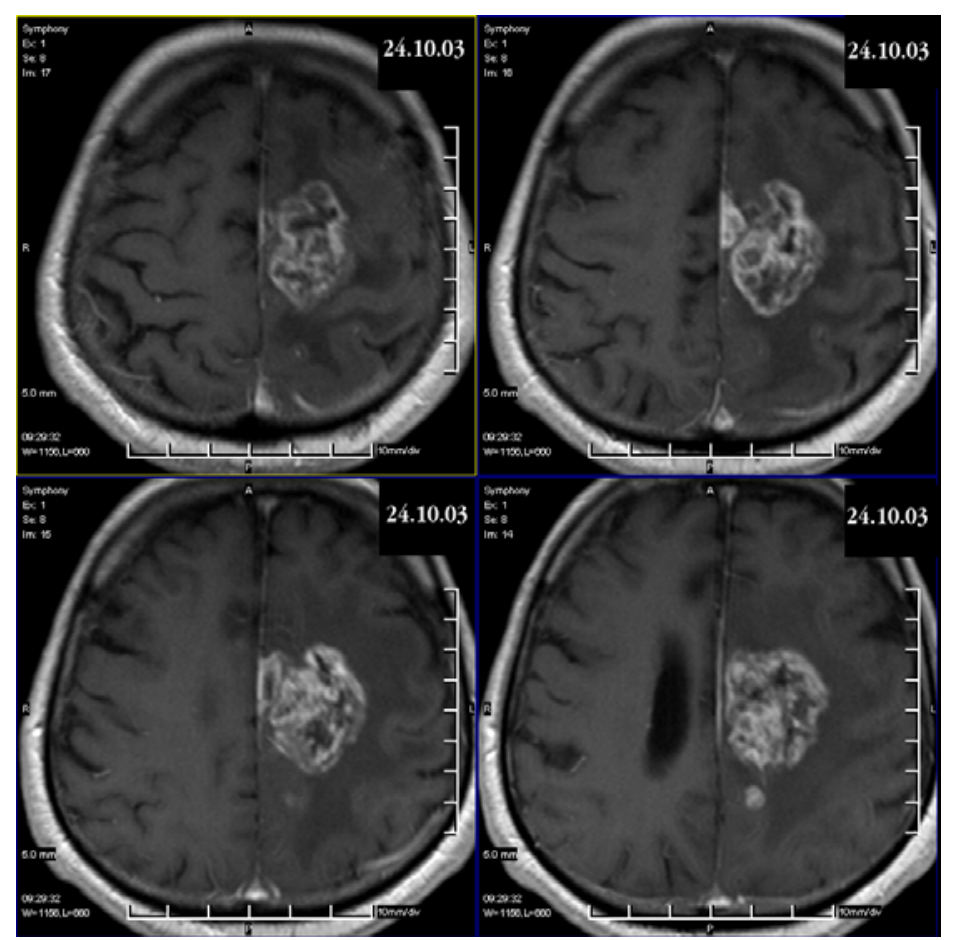

Abb.1: Patient mit links frontalem Glioblastomrezidiv 8 Monate nach subtotaler Resektion und Bestrahlung

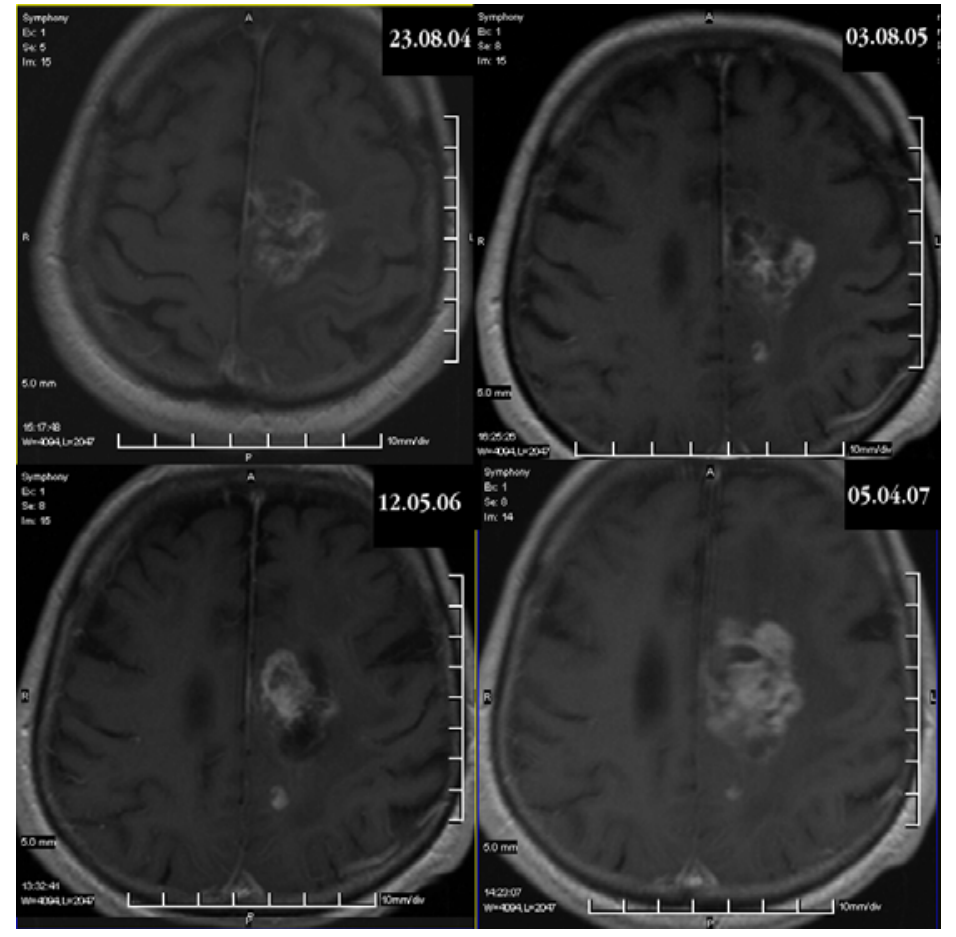

Abb. 2: Auszüge aus 3-4-monatigen MRT-Verlaufskontrollen unter Temozolomid-Chemotherapie mit bildmorphologischem und klinischem Tumorprogress schließlich im April 2007. 


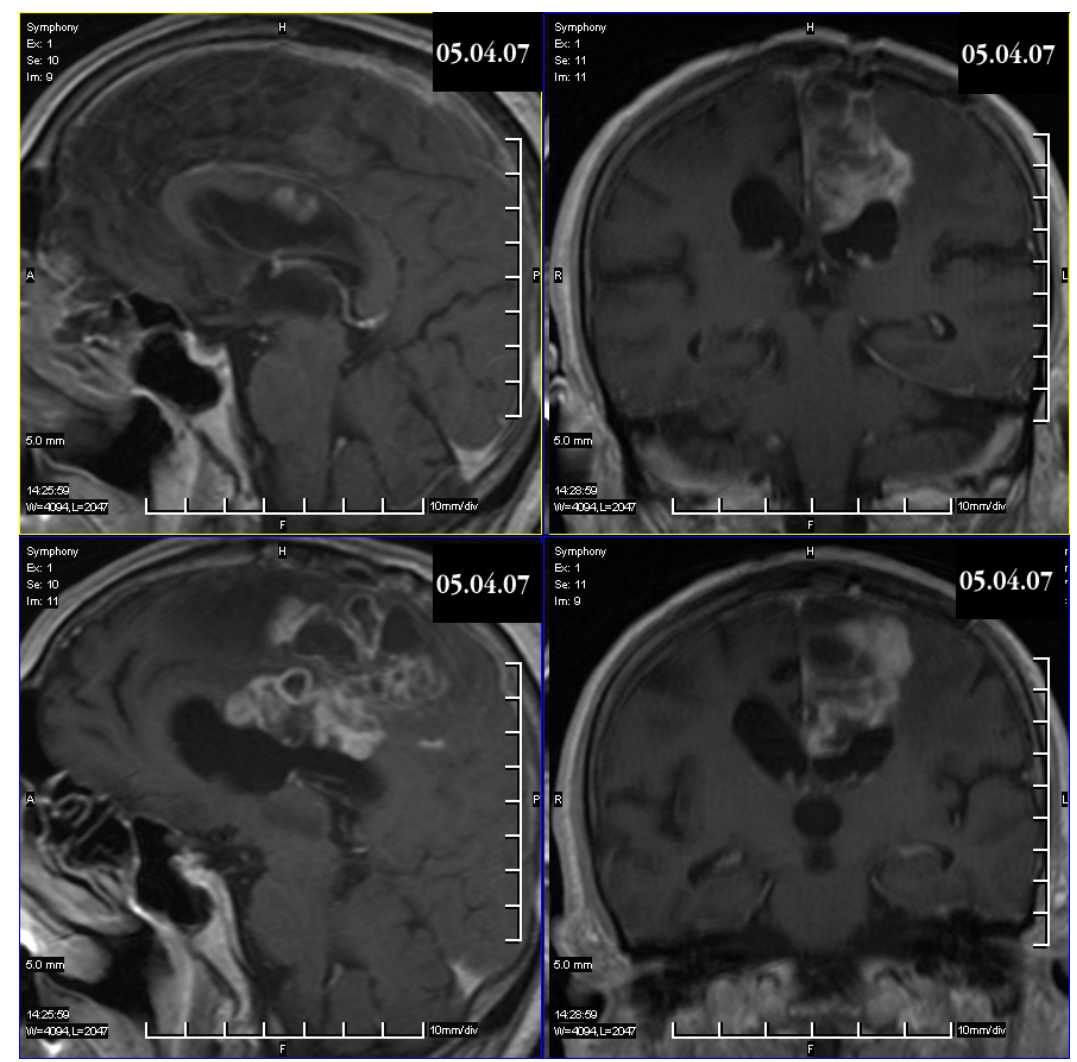

Abb. 3: Progredienter Rezidivtumor nach 20 abgeschlossenen Zyklen Temozolomid-Chemotherapie in kontrastgestützter sagittaler und koronarer Schichtung.

\subsection{Fragestellung}

Die aktuelle Therapie von Patienten mit pathohistologisch gesichertem Glioblastom wird heute vorgegeben durch die aktuelle Studienlage groß angelegter, prospektiver, multizentrischer Untersuchungen eines meist randomisierten Patientenkollektivs.

Vorerkrankungen, präoperativer klinischer Zustand, Alter des Patienten und Operabilität des Tumors sind im klinischen Alltag entscheidende Einflusskriterien für die Planung der individuellen chirurgischen und adjuvanten Therapie. Die klinischen Verläufe von Glioblastompatienten nach operativer und adjuvanter Therapie bedürfen einer möglichst detaillierten und vollständigen Dokumentation, um retrospektiv die Profitabilität der therapeutischen Maßnahmen beurteilen und zukünftige, neue Therapieformen vergleichbar machen zu können. Bei der hier vorliegenden Untersuchung handelt es sich um eine retrospektive Analyse zeitgemäßer Therapiestrategien eines nicht randomisierten Patientenkollektivs aus der Universitätsklinik Göttingen über einen Zeitraum von 7 Jahren. Es wurden sämtliche aktenkundige Patienten in die Untersuchung eingeschlossen, die in den Jahren 1998 bis 2004 an einem in Göttingen erstmals histologisch gesicherten Glioblastom erkrankten. Neben den potenziell prognostischen Einflussfaktoren, wie Alter und klinischer Zustand der Patienten, sollten die verschiedenen therapeutischen Strategien wie Resektionsausmaß, Strahlen- 
therapie und Art der Chemotherapie, bezogen auf des Gesamtüberleben und das progressfreie Überleben, untersucht werden. Durch eine systematische Datenkollektion und Verlaufsrekonstruktion, anhand einer hierfür entwickelten Tumorpatientendatenbank, wurden verschiedene jeweils zeitgemäße Therapieregime hinsichtlich ihrer Effektivität untereinander verglichen und dabei die individuellen Einflussfaktoren, Alter und klinischer Ausgangszustand, mitberücksichtigt. Im Resultat soll sich zeigen, inwieweit international empfohlene und anerkannte Therapieformen, die anhand eines randomisierten Patientenkollektivs entwickelt wurden, im klinischen Alltag umsetzbar sind, und ob die Wirksamkeit vergleichbar ist. Es sollte auf diese Weise eine institutionelle Reevaluation und Qualitätskontrolle gängiger Therapieformen des Glioblastoma multiforme in Göttingen entstehen.

\section{Patienten und Methoden}

\subsection{Das Patientenkollektiv}

Die Untersuchung wurde als retrospektive Datenanalyse aller, im Zeitraum von 1998 bis 2004 in der Klinik für Neurochirurgie der Universitätsmedizin Göttingen behandelten Patienten, mit pathohistologisch gesichertem Glioblastoma multiforme WHO Grad IV, konzipiert.

Bei fast allen Patienten wurden die primäre Operation und damit die Diagnosesicherung in Göttingen durchgeführt. Nur ein sehr kleiner Teil der Patienten unterzog sich primär einer auswärtigen operativen Therapie. Die adjuvanten radiotherapeutischen und chemotherapeutischen Maßnahmen erfolgten ausnahmslos ambulant oder stationär in der Universitätsklinik Göttingen, so auch alle auf die Erstoperation folgenden neurochirurgischen Eingriffe. Es wurden alle Patienten mit nachgewiesenem Glioblastom in die Datenanalyse eingeschlossen, unabhängig von Alter, Geschlecht, Vorerkrankungen oder anderer prognostischer Einflussfaktoren. Es handelte sich also um ein unselektiertes Patientenkollektiv, wie es sich im klinischen Alltag einer neurochirurgischen Klinik präsentiert. Nicht erfasst wurden Patienten mit bildmorphologischem Verdacht auf einen hirneigenen Tumor, die keine Sicherung der Diagnose durch eine operative Maßnahme erfahren haben.

\subsection{Patientendaten}

Durch Recherche im Zentralarchiv der Universitätsklinik Göttingen wurden aus den Patientenakten Daten zur Rekonstruktion des Krankheits- und Therapieverlaufes, bis 
hin zum Tod der Patienten oder bis zum Zeitpunkt des letzten klinischen Kontaktes, erfasst (Tabelle 1). Im Falle von fehlenden oder aus den Akten allein nicht ersichtlichen Informationen, wie Todesdaten oder klinischen und radiologischen Befunden, wurden die entsprechenden Einwohnermeldeämter oder Hausärzte per Anschreiben um Auskunft gebeten. Auf persönliche Anfragen bei Patienten und Hinterbliebenen wurde grundsätzlich verzichtet.

\subsection{Datenschutz}

Die Akteneinsicht zum Zwecke der wissenschaftlichen retrospektiven Analyse wurde durch den Direktor der Abteilung Neurochirurgie, Prof. Dr. med. Veit Rohde, und durch den die Promotionsarbeit betreuenden leitenden Oberarzt der Abteilung Neurochirurgie, Professor Dr. med. Alf Giese, schriftlich bewilligt. Alle erfassten Patientendaten wurden zum Schutz vor Fremdzugriff auf Festplatten von Computersystemen ohne Zugang zum Internet gespeichert und zusätzlich separat auf vorgefertigten Datenerfassungsbögen in Papierform dokumentiert.

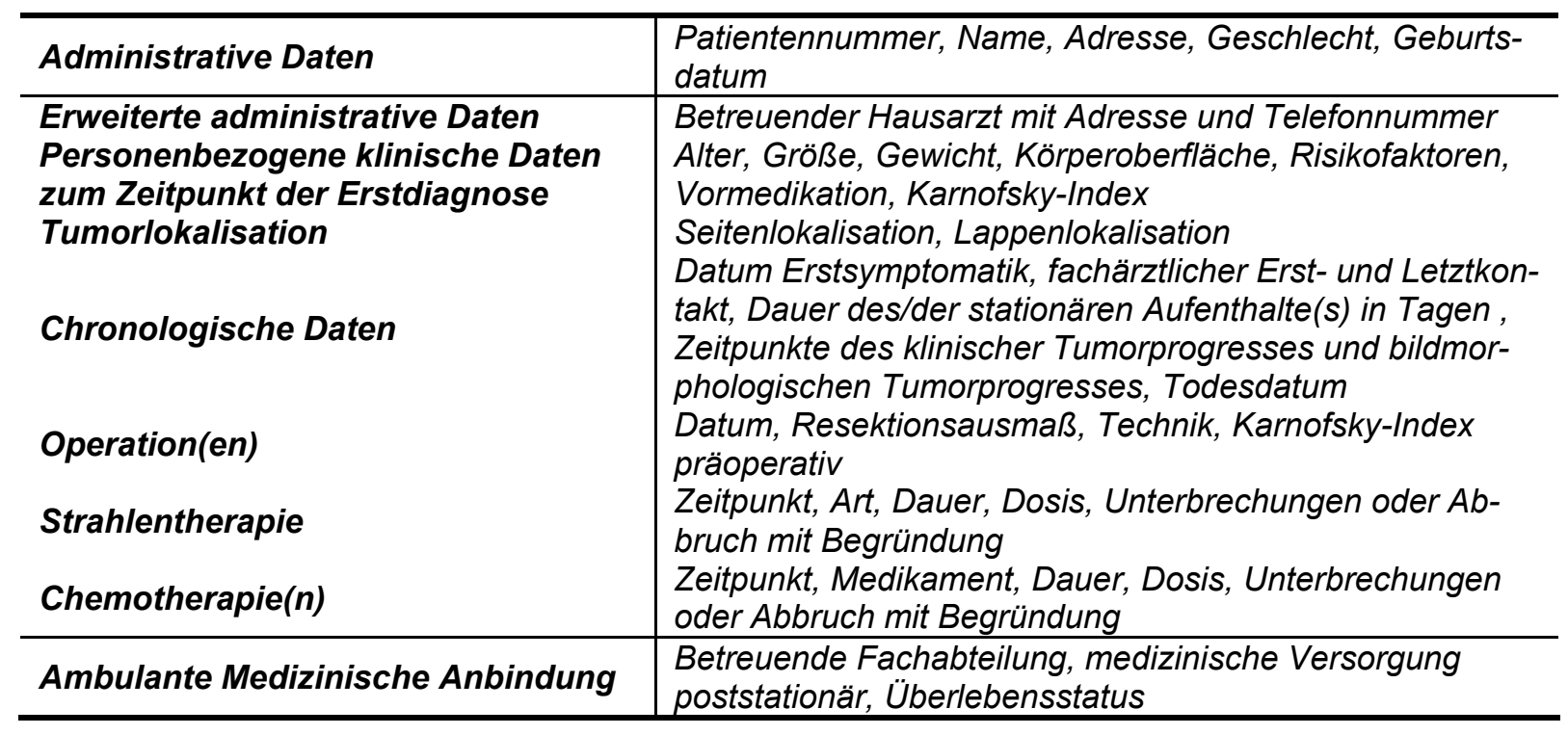

Tabelle 1: Datenerhebung aus den neurochirurgischen Patientenakten

\subsection{Datenerfassung über eine formularbasierte Datenbank}

Um die erhobenen Daten in eine korrelierbare Struktur einzugruppieren und eine suffiziente statistische Auswertbarkeit zu gewährleisten, erfolgte die Datenerhebung auf der Basis einer formulargestützten MS Access-Datenbank. Diese wurde durch Mitarbeiter der Abteilung Neurochirurgie (Dr. med. Hans Christoph Bock) und der Abteilung Anästhesiologie, Rettungs- und Intensivmedizin (PD Dr. med. Jose Hinz) der 
Universitätsklinik Göttingen entwickelt. Die Datenerhebung und Dokumentation konnte so mit Hilfe von systematisierten Formularen erfolgen, welche wiederum auf zuvor erstellten Tabellen mit umfangreicher Verknüpfung untereinander basierten.

\subsubsection{Formulare}

Für jeden Patienten wurde sowohl ein Stammdatensatz als auch ein klinischer Verlaufsdatensatz mit den entsprechenden chronologischen Daten der adjuvanten Therapiemaßnahmen und den Progresszeitpunkten angelegt. Um vergleichbare Datensätze zu generieren, wurden Dokumentationskriterien als analoge Auswahllisten ,drop-down'-Menüs erstellt, z.B. eine Auswahl von Chemotherapien oder Formen der Strahlentherapie.

Über das Startformular gelangt man über verschieden Schaltflächen in die einzelnen Unterformulare, welche aus Daten der einzelenen Basistabellen bestehen und untereinander verknüpft sind.

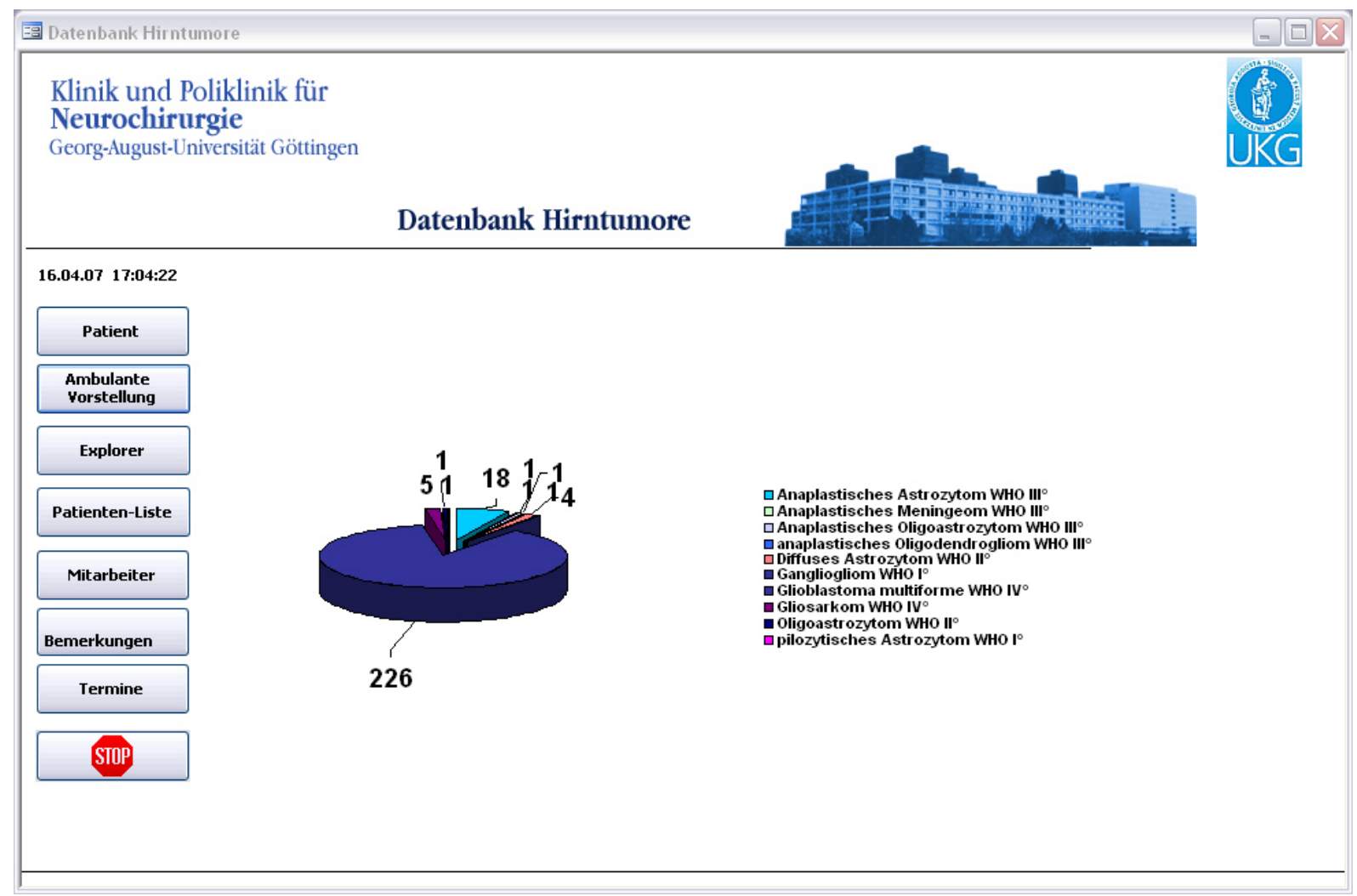

Abb.4: Startbildschirm der Datenbank mit ständig aktualisiertem Stand der Datenerfassung

Durch die Indizierung der Patienten nach individuellen Patientennummern mit Prüfziffern wurde sichergestellt, dass für jeden Patienten nur jeweils ein Stammdatensatz angelegt werden kann und Verwechslungen z.B. durch Namensänderungen im Verlauf der Erkrankung ausgeschlossen sind. Über das Stammdatenformular können 
alle Informationen zum Patienten und den Operationen dokumentiert und eingesehen werden. Verknüpfte Schaltflächen ermöglichen dann die Dokumentation der durchgeführten adjuvanten Therapiemaßnahmen und zeitlichen Verläufe der Tumorprogression zu jeder durchgeführten Tumoroperation.

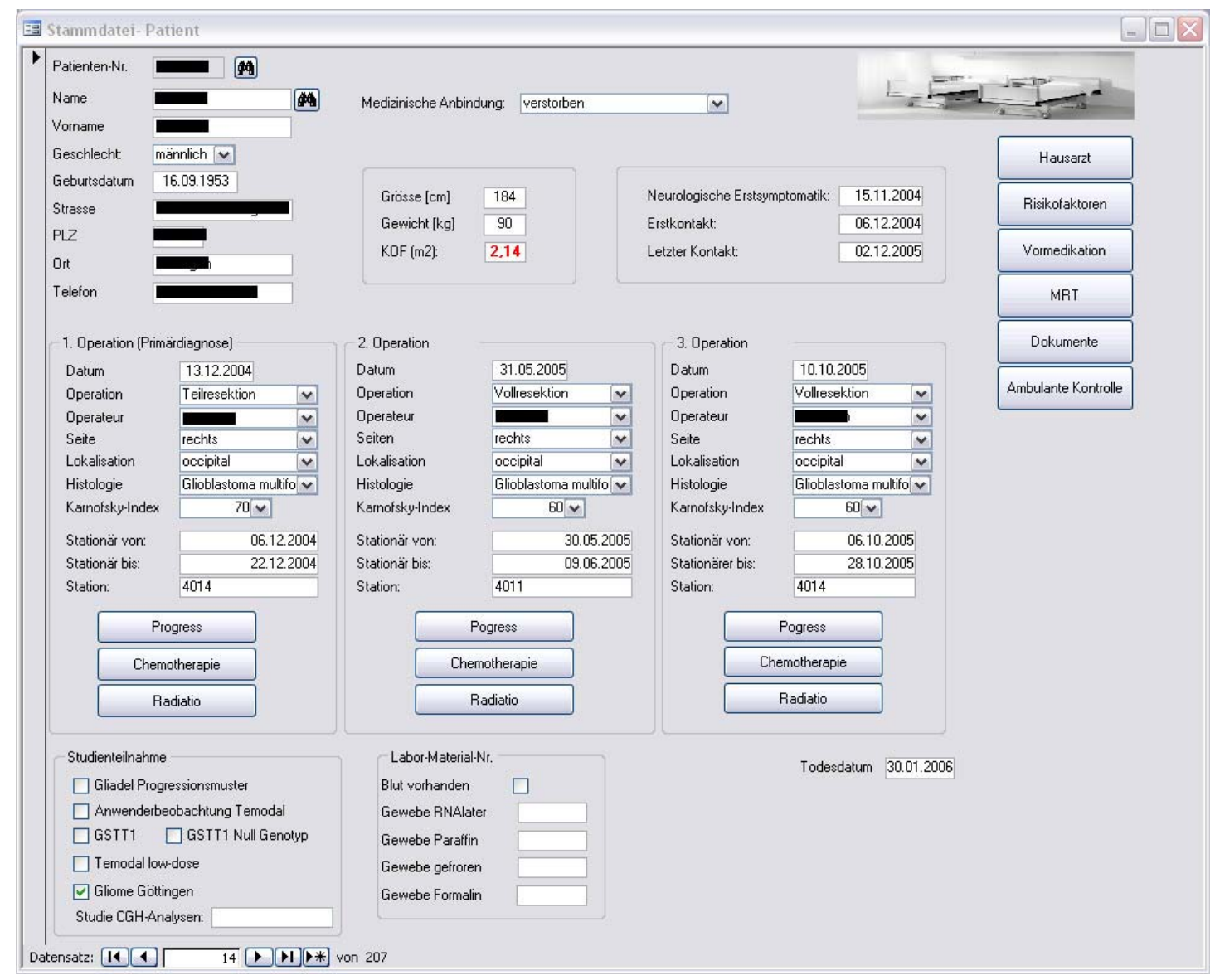

Abb.5: Beispiel für ein Formular der ,Stammdatei Patient', anonymisiert

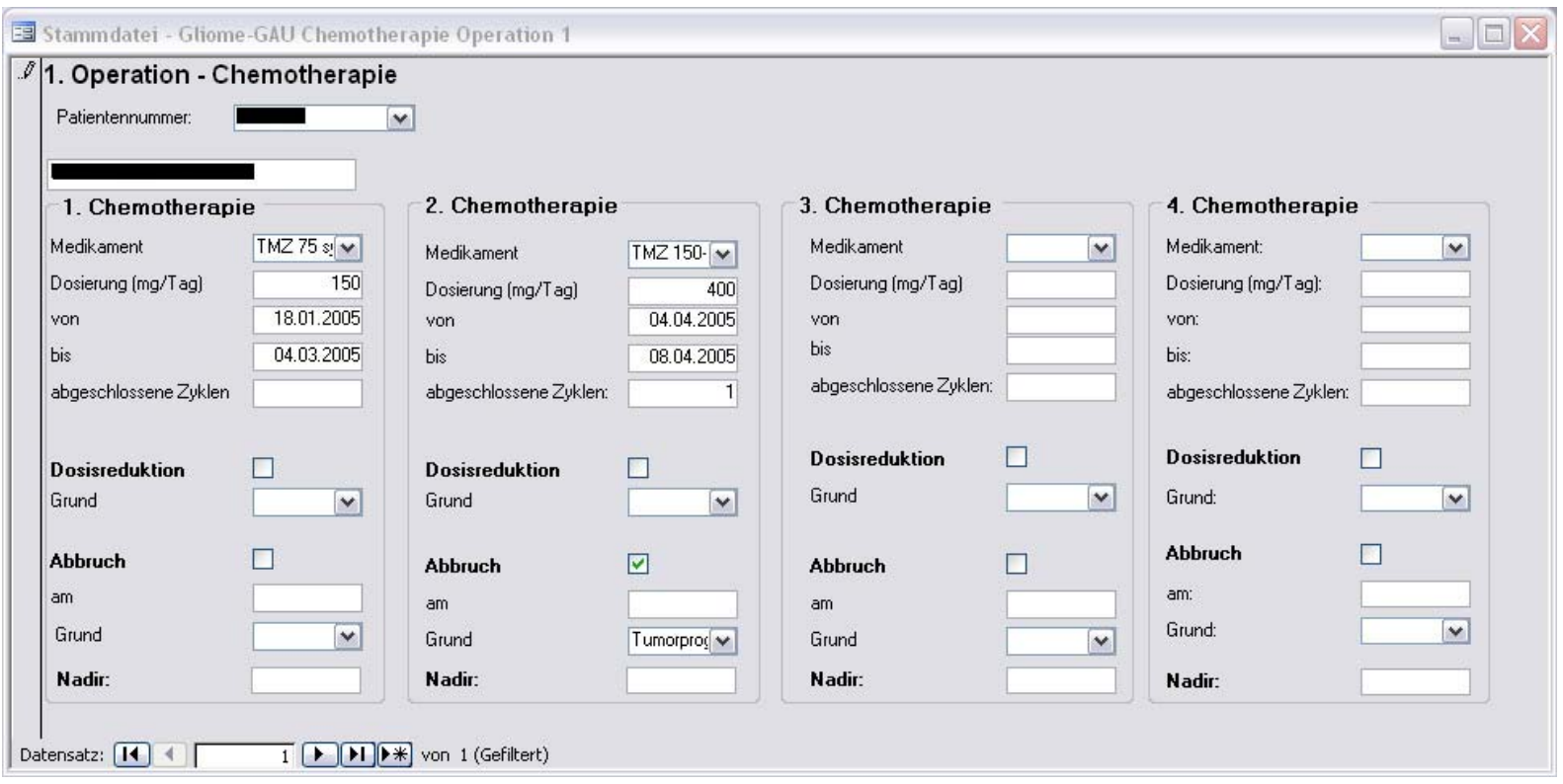

Abb.6: Beispiel für ein Formular, Chemotherapie nach Operation 1' (anonymisiert) 

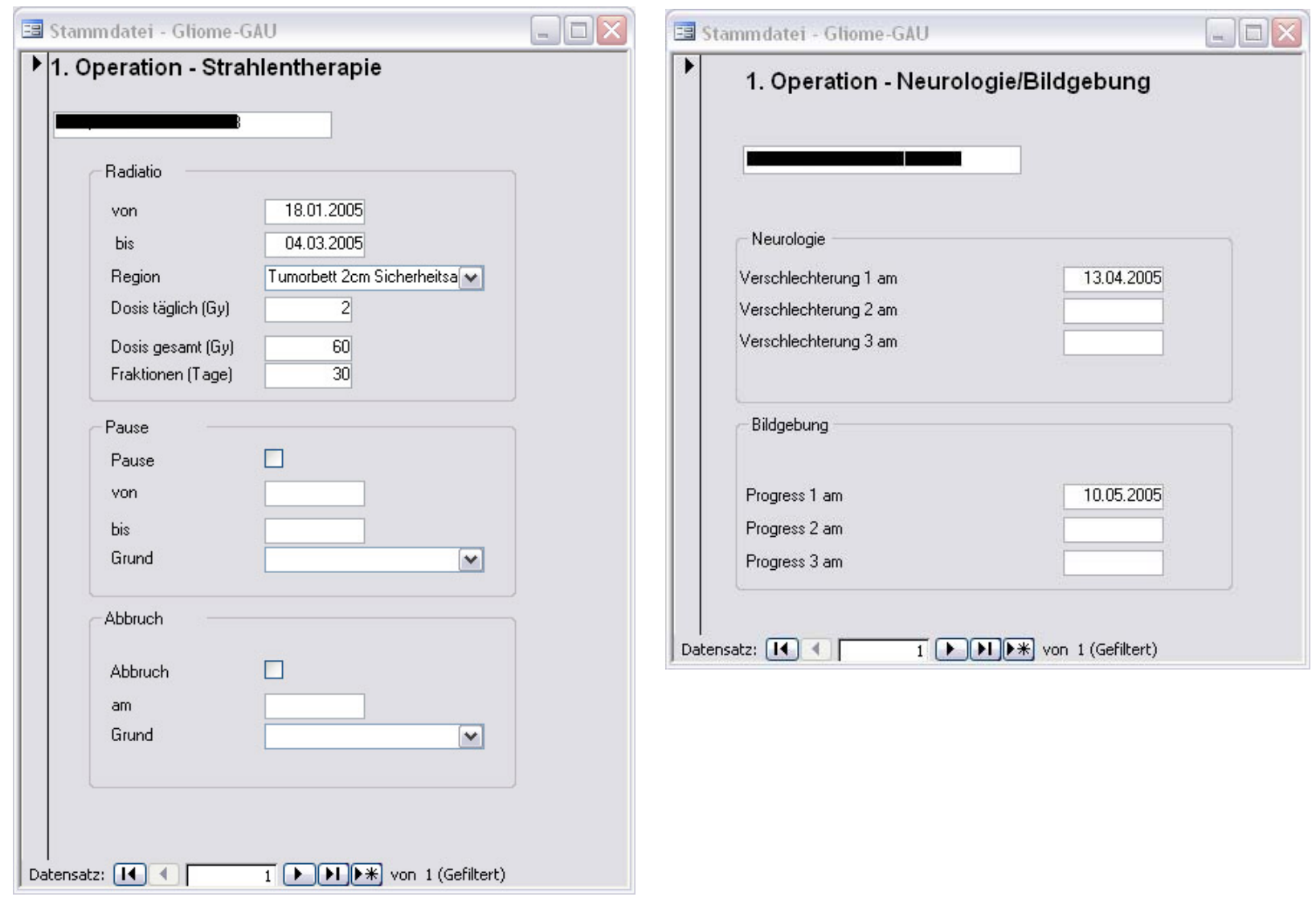

Abb.7 und 8: Beispiel für ein Formular, Strahlentherapie nach Operation 1' und ,klinischer' und ,bildgebender' Tumor-Progress nach ,Operation 1' (anonymisiert)

\subsection{Datenauswertung}

Alle erhobenen Daten wurden in einer nach den individuellen Patientennummern indizierten Tabelle gespeichert. Da die Vielzahl der erhobenen Daten als Darstellung und Auswertung in einer einzelnen Tabelle mangels Übersichtlichkeit nicht möglich wäre, bedienten wir uns der Möglichkeit des Abfragegenerators von MS Access. Hier können die jeweils gewünschten Datensätze aus der Gesamtdatenbank gefiltert werden. Wir selektierten aus allen erfassten Patientendaten nur die für unsere Untersuchung notwendigen Daten und konnten nach definierbaren Kriterien Patientengruppen identifizieren und miteinander vergleichen. Alle in MS Access generierten Tabellen wurden mit MS Excel weiterbearbeitet und mit verschiedenen Statistikprogrammen ausgewertet. Eine gute Lösung fand sich in der Anwendung von WinStat (R. Fitch Software, Bad Krozingen, Deutschland), welche integriert in MS Excel alle notwendigen statistischen Auswertungen ermöglichte. 


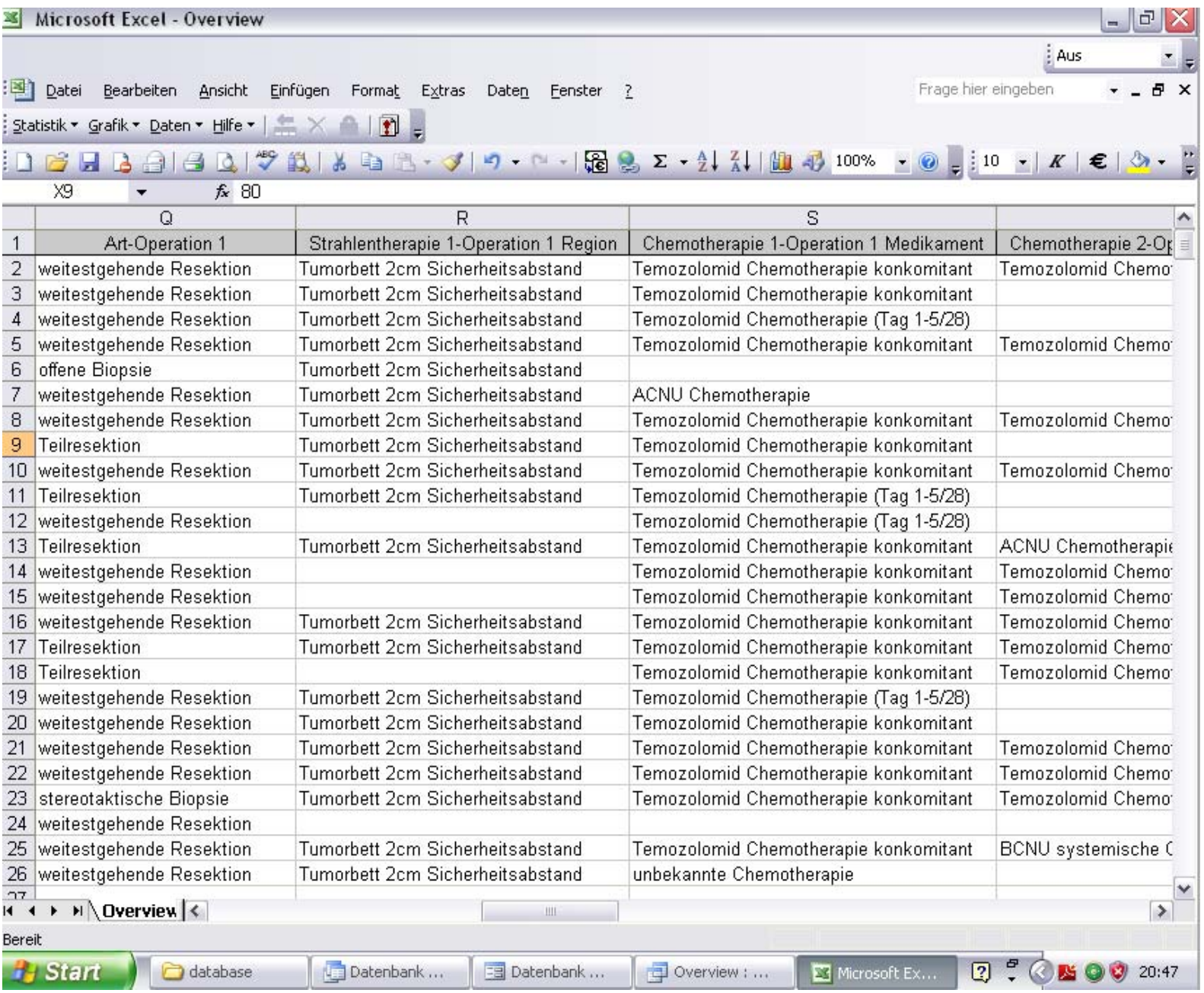

Abb. 9: Aus der Tabelle aller erhobenen Patientendaten können mittels eines Abfragegenerators beliebige Daten aus der Datenbank gefiltert und dann über Tabellenkalkulation und Statistiksoftware ausgewertet werden.

\subsubsection{Erstellung von Diagrammen}

Die aus den in MS Access generierten Datensätze (Auswahlabfragen) wurden in Tabellenform direkt mit MS Excel bearbeitet. Über die in Excel integrierten Funktionen wurden die Tabellen in Diagramme überführt. Auch die Berechnung von statistischen Werten, wie Mittelwerte, Mediane und Standardabweichungen erfolgten über die in MS Exce/ integrierten Funktionen.

\subsubsection{Erstellung von Survivalanalysen und Progressanalysen}

Um eine Analyse der Überlebenswahrscheinlichkeit einer Patientengruppe durchzuführen, wurde das in MS Excel integrierte Statistikprogramm Winstat ${ }^{\circledR}$ verwendet. Für alle Survivalanalysen, die ein Zusatzkriterium als Variable beinhalteten (z.B. Survival nach Resektionsausmaß), mussten zuvor entsprechende Klassendefinitionen erfolgen (z.B. Klasse Resektionsausmaß OP1: Biopsie, subtotal, makroskopische Vollresektion). 


\begin{tabular}{|c|c|c|c|c|c|}
\hline AA & $\mathrm{AB}$ & $A C$ & $\mathrm{AD}$ & AE & AF \\
\hline Datum des Todes & Überlebt bis (Datum) & Alter ED(Jahre) & Überlebenszeit gesamt(Monate) & Tod & OP-Art \\
\hline 10.12 .2001 & 10-Dez-01 & 77 & 4 & 1 & $\mathrm{PE}$ \\
\hline 16.09 .1998 & 16-Sep-98 & 70 & 5 & 1 & $\mathrm{PE}$ \\
\hline 27.04 .2004 & 27-Apr-04 & 58 & 20 & 1 & $\mathrm{PE}$ \\
\hline 02.07 .2003 & 02-Jul-03 & 75 & 3 & 1 & $\mathrm{PE}$ \\
\hline 08.02 .2003 & 08-Feb-03 & 41 & 14 & 1 & $\mathrm{PE}$ \\
\hline 14.02 .2005 & 14-Feb-05 & 41 & 15 & 1 & $\mathrm{PE}$ \\
\hline 20.03 .1999 & 20-Mrz-99 & 59 & 8 & 1 & $P E$ \\
\hline 26.06 .1999 & 26-Jun-99 & 66 & \multirow{2}{*}{ Klassendefinition } & \multirow{2}{*}{$x$} & PE \\
\hline 10.12 .2000 & 10-Dez-00 & 71 & & & $\mathrm{PE}$ \\
\hline 09.07 .1999 & 09-Jul-99 & 74 & \multirow{7}{*}{$\begin{array}{l}\text { Variable: } \\
\text { OP-Art }\end{array}$} & \multirow[b]{2}{*}{ ok } & $\mathrm{PE}$ \\
\hline 02.10 .1998 & $02-0 \mathrm{kt}-98$ & 55 & & & $\mathrm{PE}$ \\
\hline 15.08 .1999 & 15-Aug-99 & 74 & & \multirow{2}{*}{ Abbrechen } & subtotal \\
\hline 14.10.1999 & 14-Okt-99 & 63 & & & subtotal \\
\hline 27.03 .2001 & 27-Mrz-01 & 62 & & \multirow{3}{*}{ ? } & subtotal \\
\hline 07.03 .2002 & 07-Mrz-02 & 63 & & & subtotal \\
\hline 28.08 .2004 & 28-Aug-04 & 73 & & & subtotal \\
\hline 30.01 .2006 & 30-Jan-06 & 51 & 13 & 1 & subtotal \\
\hline 19.03 .2003 & 19-Mrz-03 & 74 & 6 & 1 & subtotal \\
\hline 10.09 .2003 & 10-Sep-03 & 72 & 2 & 1 & subtotal \\
\hline 06.01 .2002 & 06-Jan-02 & 58 & 3 & 1 & subtotal \\
\hline 06.03 .2000 & $06-$ Mrz-00 & 55 & 16 & 1 & subtotal \\
\hline 02.07 .2003 & 02-Jul-03 & 59 & 18 & 1 & subtotal \\
\hline 24.10.1999 & 24-Okt-99 & 62 & 12 & 1 & subtotal \\
\hline כ7 ח1 כחกא & $27 \ln n \cap 5$ & $A E$ & $a$ & 1 & nuibtental \\
\hline
\end{tabular}

Abb. 10: Klassendefinition für korrelierende Survivalanalyse

Nach erfolgter Klassendefinition konnte nun die Survivalanalyse anhand der spezifischen Variable (in diesem Falle: Resektionsausmaß = OP-Art) durchgeführt werden.

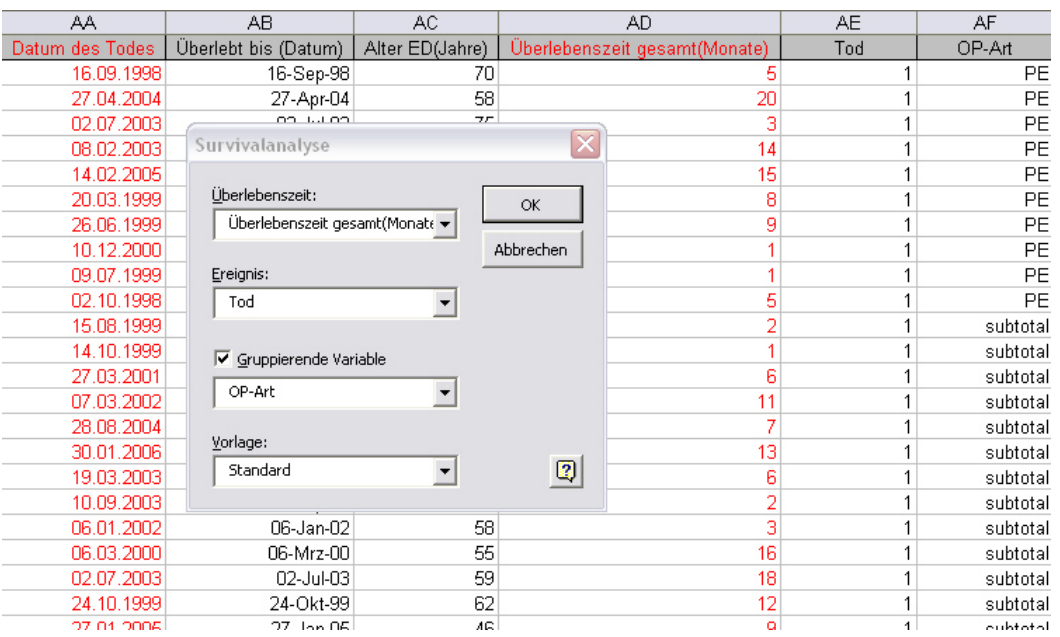

Abb. 11: Parameterdefinition für Survivalanalyse mit gruppierender Variable

Im Ergebnis wurde eine Kaplan-Meyer-Kurve generiert, in der für jede gruppierende Variable die entsprechenden Wahrscheinlichkeiten dargestellt werden und schließlich auch statistische Werte, wie P-Wert und Chi-Quadrat-Test beinhaltete. Durch grafische Aufarbeitung konnten dann die Überlebenswahrscheinlichkeiten neben der Tabelle auch aus der graphischen Darstellung entnommen werden. 

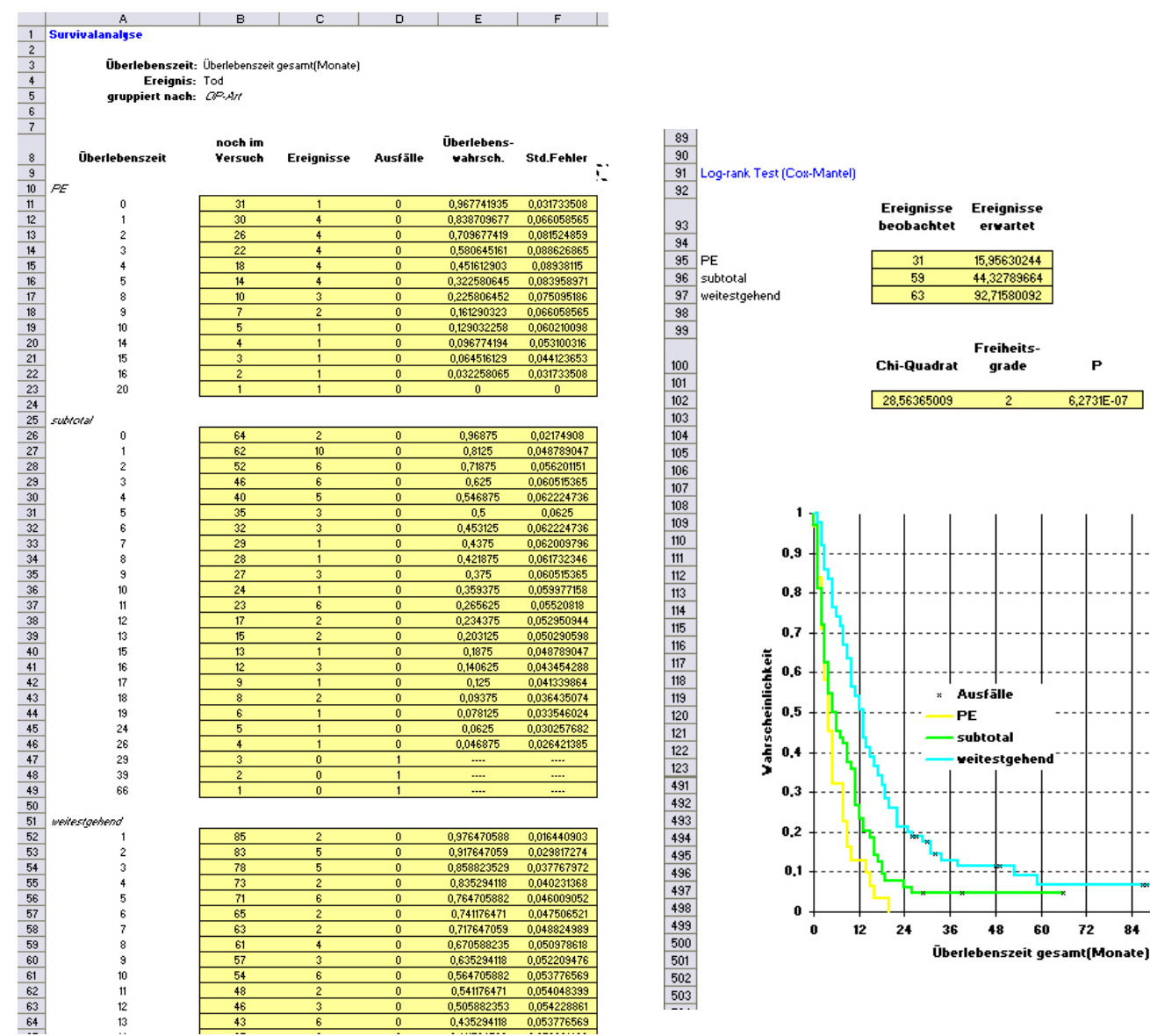

Abb. 12: Berechnung der Überlebenswahrscheinlichkeiten mit Winstat

Zur Erstellung von Progressionsanalysen wurde prinzipiell das gleiche Vorgehen gewählt, nur wurde hier die progressfreie (klinisch oder bildmorphologisch) Überlebenszeit anstelle der Gesamtüberlebenszeit untersucht. Bei der Analyse des klinischen Progresses wurden nur Kriterien berücksichtigt, bei denen, im Rahmen einer Wiedervorstellung in der neurochirurgischen oder neurologischen Klinik, in der Patientenakte ein neurologischer Status quantifizierbar dokumentiert wurde. Auch bei der Analyse der bildmorphologischen Progression wurden nur Patienten in die Untersuchung eingeschlossen, bei denen von einem Neuroradiologen, im Vergleich zu den Voraufnahmen, Kriterien des Progresses festgestellt und in schriftlicher Befundform in der Patientenakte dokumentiert wurden. Insbesondere in der ersten Hälfte des Untersuchungszeitraumes erfolgten MRT-Kontrollen mit intravenösem Kontrastmittel nicht routinemäßig im dreimonatigen Abstand, sondern häufig erst im Rahmen einer klinischen Verschlechterung des Patienten. Analysiert wurden alle Datensätze. Zum einen die Patienten, bei denen sowohl klinische und bildmorphologische Progresse eindeutig in der Patientenakte dokumentiert waren als auch Patienten ohne dokumentierten Progress. Hier wurde entsprechend das Todesdatum als definitives Progressdatum gewertet. 


\section{Ergebnisse}

\subsection{Darstellung des Gesamtkollektivs}

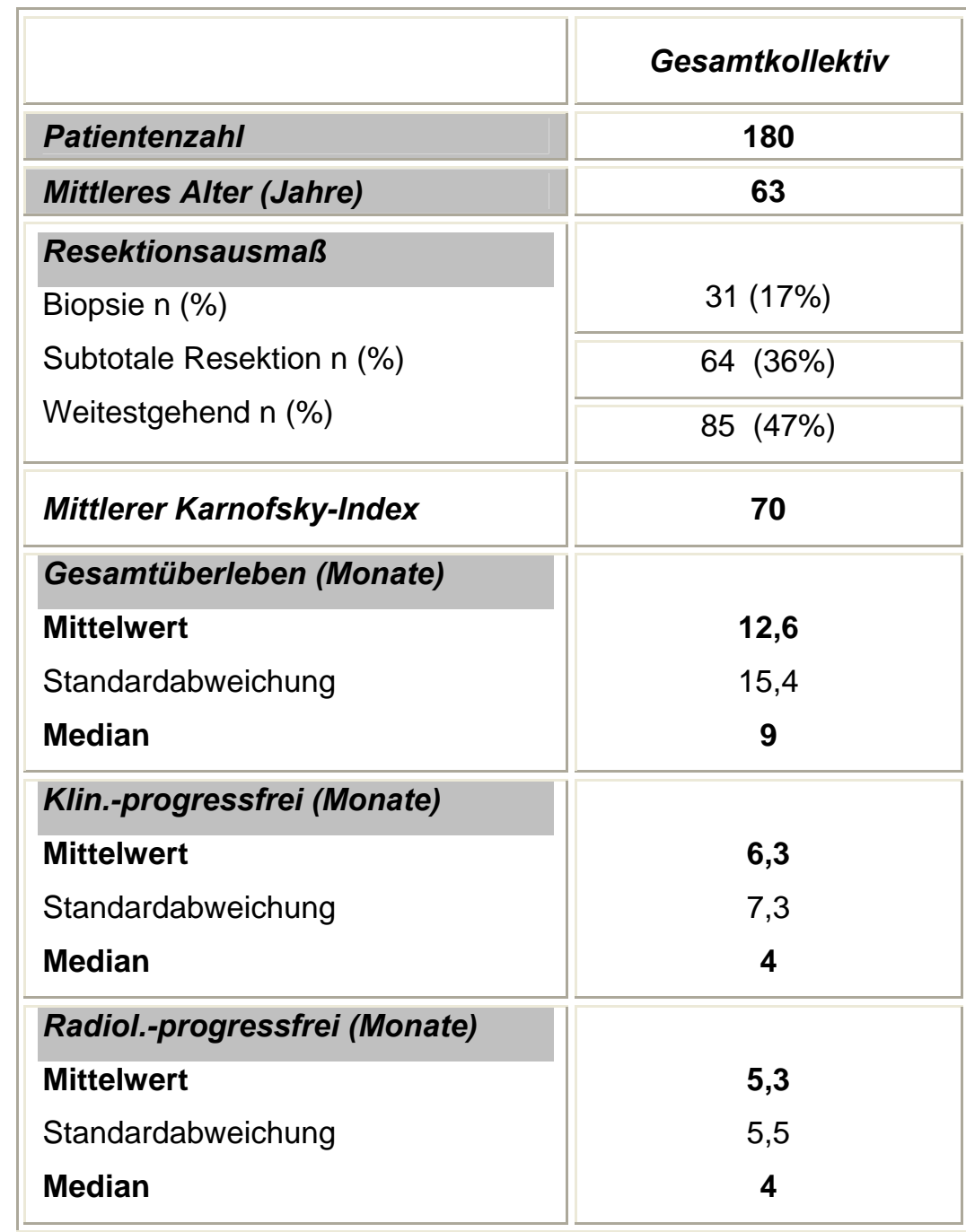

Tabelle 2: Tabellarische Darstellung des Gesamtkollektivs 


\begin{tabular}{|c|c|c|c|c|c|c|c|}
\hline Untersuchungsjahr & 1998 & 1999 & 2000 & 2001 & 2002 & 2003 & 2004 \\
\hline Patientenzahl & 33 & 21 & 26 & 24 & 30 & 24 & 22 \\
\hline Mittlerer Karnofsky-Index & 70 & 70 & 60 & 70 & 60 & 70 & 70 \\
\hline Mittleres Alter (Jahre) & 64 & 62 & 61 & 60 & 65 & 64 & 62 \\
\hline \multicolumn{8}{|l|}{ Resektionsausmaß } \\
\hline Biopsie $n(\%)$ & $6(18)$ & $3(14)$ & $5(19)$ & $6(25)$ & $4(13)$ & $5(21)$ & $2(9)$ \\
\hline Subtotale Resektion n (\%) & $10(30)$ & $7(34)$ & $9(35)$ & $9(37)$ & $11(37)$ & $9(37)$ & $9(41)$ \\
\hline Weitestgehend $n$ (\%) & $17(52)$ & $11(52)$ & $12(46)$ & $9(37,5)$ & $15(50)$ & $10(42)$ & $11(50)$ \\
\hline \multicolumn{8}{|l|}{ Gesamtüberleben (Monate) } \\
\hline Mittelwert & 12,3 & 16,2 & 10,2 & 12,4 & 10,6 & 11,9 & 16 \\
\hline Standardabweichung & 18,4 & 25,2 & 11,3 & 14,7 & 10,4 & 14,5 & 9,5 \\
\hline Median & 7 & 6 & 9 & 8,5 & 9 & 5 & 14,5 \\
\hline \multicolumn{8}{|l|}{ Klin.-progressfrei (Monate) } \\
\hline Mittelwert & 4,4 & 6,6 & 6,9 & 6,7 & 6,7 & 5,4 & 8,4 \\
\hline Standardabweichung & 4,8 & 8 & 10,5 & 9,6 & 5,8 & 4,5 & 6,3 \\
\hline Median & 3,4 & 3 & 4,1 & 2,9 & 5 & 3,3 & 7,5 \\
\hline \multicolumn{8}{|l|}{ Radiol.-progressfrei (Monate) } \\
\hline Mittelwert & 4,5 & 6,2 & 6,7 & 3,5 & 5,3 & 4,7 & 6,8 \\
\hline Standardabweichung & 3,6 & 7 & 10 & 2,5 & 4,2 & 3,2 & 4,2 \\
\hline Median & 3,5 & 3,9 & 4,6 & 3 & 4,6 & 3,5 & 6,6 \\
\hline
\end{tabular}

Tabelle 3: Tabellarische Darstellung des Gesamtkollektivs aufgeschlüsselt nach den Untersuchungszeiträumen

\subsubsection{Fallzahl und Geschlechterverteilung}

Das hier untersuchte Patientenkollektiv umfasste alle Patienten mit einem, in der Universitätsklinik Göttingen, pathohistologisch nachgewiesenen neu diagnostizierten Glioblastom WHO Grad IV aus dem Zeitraum von 1998 bis einschließlich 2004. Das Gesamtkollektiv unserer Untersuchung über diesen Zeitraum von 7 Jahren bestand aus 180 ausgewerteten Fällen, davon 102 (57\%) Männer und 78 (43\%) Frauen. 21 weitere Fälle neu diagnostizierter Glioblastome konnten aufgrund unvollständiger Krankenblätter nicht in die Untersuchung einfließen. Die Anzahl der Fälle in Göttin- 
gen neu diagnostizierter Glioblastome zeigte eine geringe Variation in den einzelnen Jahren.

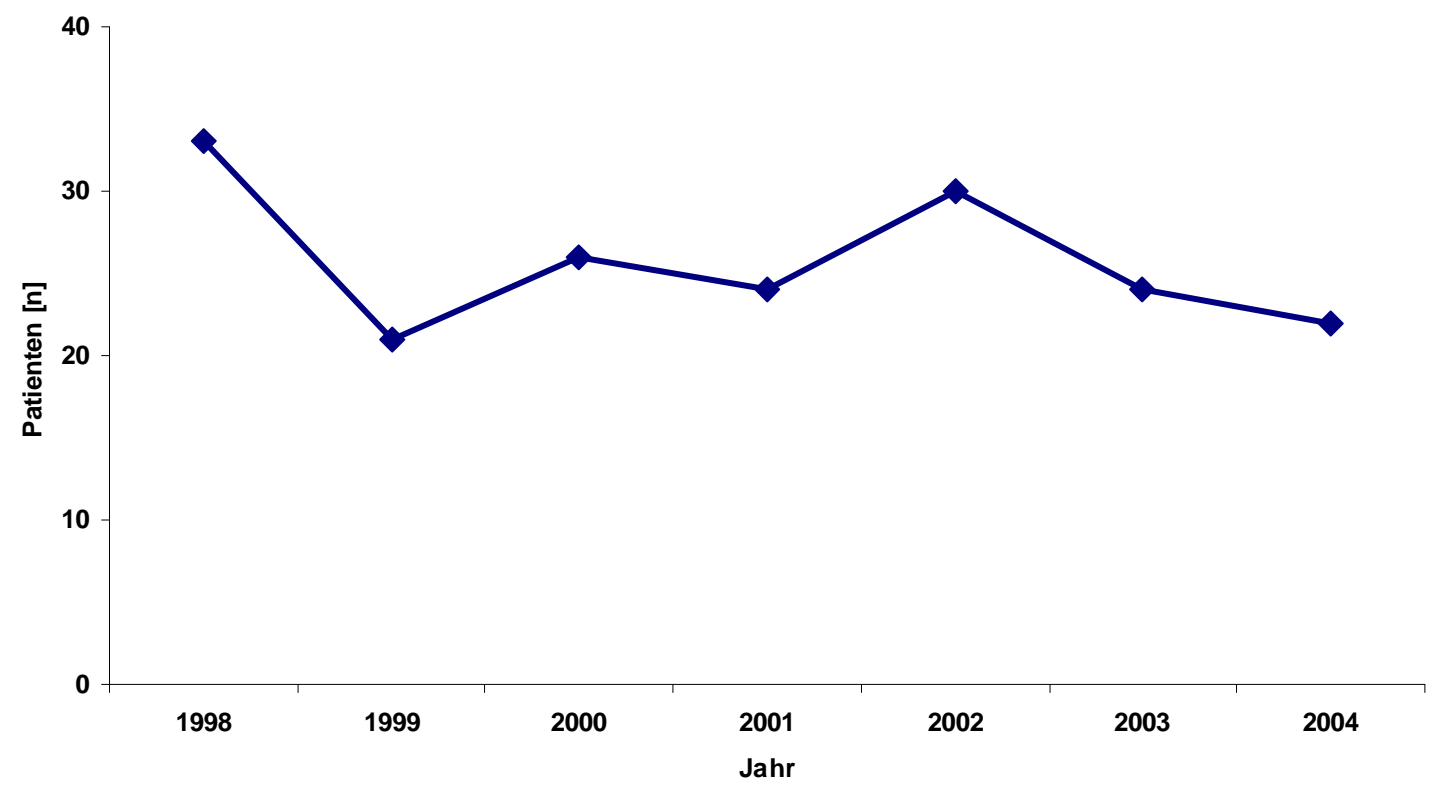

Abb.13: Anzahl pathohistologisch gesicherter, neu diagnostizierter Glioblastome (GBM) WHO Grad IV

\subsubsection{Alter}

Der jüngste Patient des Gesamtkollektivs war 30 Jahre alt, der älteste 85 Jahre. Das mittlere Alter zum Zeitpunkt der Diagnosestellung lag bei 63 Jahren. Das Durchschnittsalter zum Zeitpunkt der Diagnose variierte kaum in den verschiedenen Untersuchungszeiträumen (siehe Tab.3).

Bei der Einteilung der Patienten in Altersgruppen hatte die Gruppe 60 bis 69 Jahre mit 34\% den größten Anteil, gefolgt von der Gruppe der 70- bis 80 jährigen mit 30\%. Die Gruppe der Patienten unter 50 Jahre machte nur einen geringen Teil der Gesamtpopulation aus (Abb.14). 


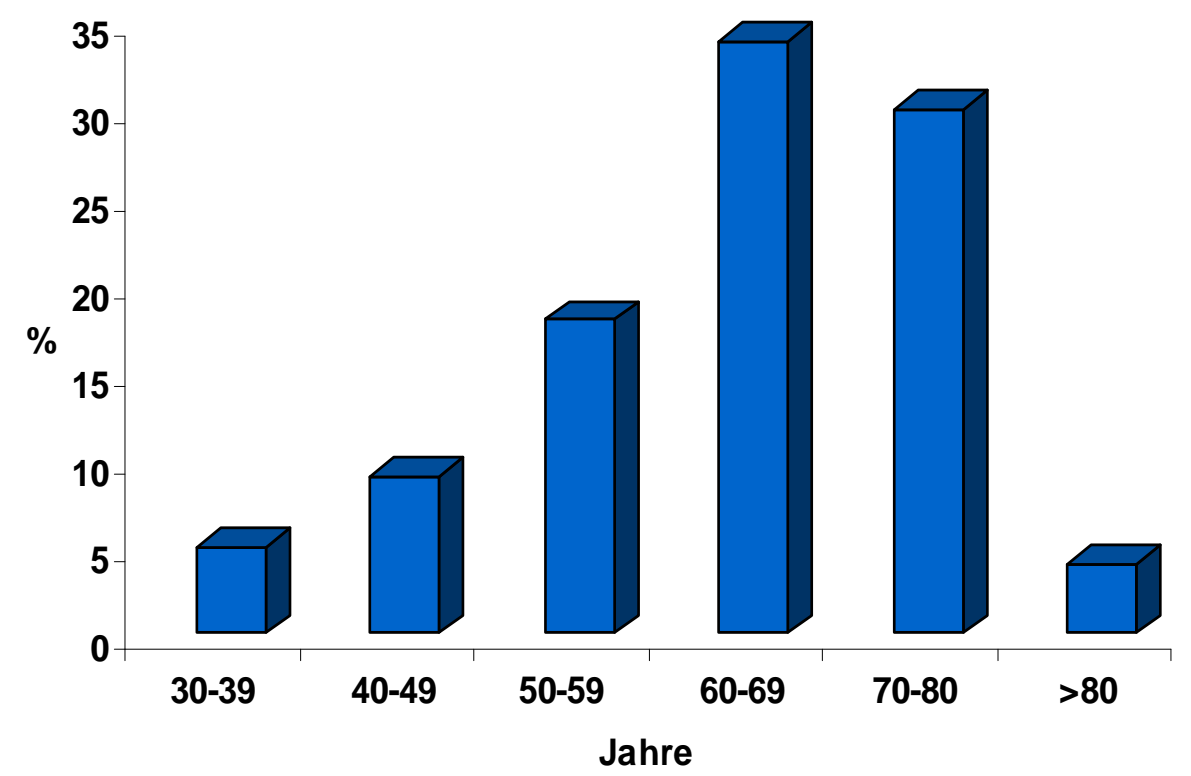

Abb.14: Altersverteilung der Gesamtpopulation von Patienten mit neu diagnostiziertem Glioblastom

\subsubsection{Initialer Karnofsky-Index}

Der Karnofsky-Index wurde retrospektiv aus dem klinischen Aufnahmebefund vor der ersten operativen Intervention ermittelt. Die ermittelten Werte zeigten eine Spannbreite von 20 Punkten (Krankenhauspflege und supportive Maßnahmen erforderlich) bis maximal 90 Punkte (minimale Krankheitssymptome). Im Mittel lag der KarnofskyIndex vor Erstoperation bei 70 Punkten (eingeschränkte Leistungsfähigkeit, arbeitsunfähig, der Patient kann sich alleine versorgen).

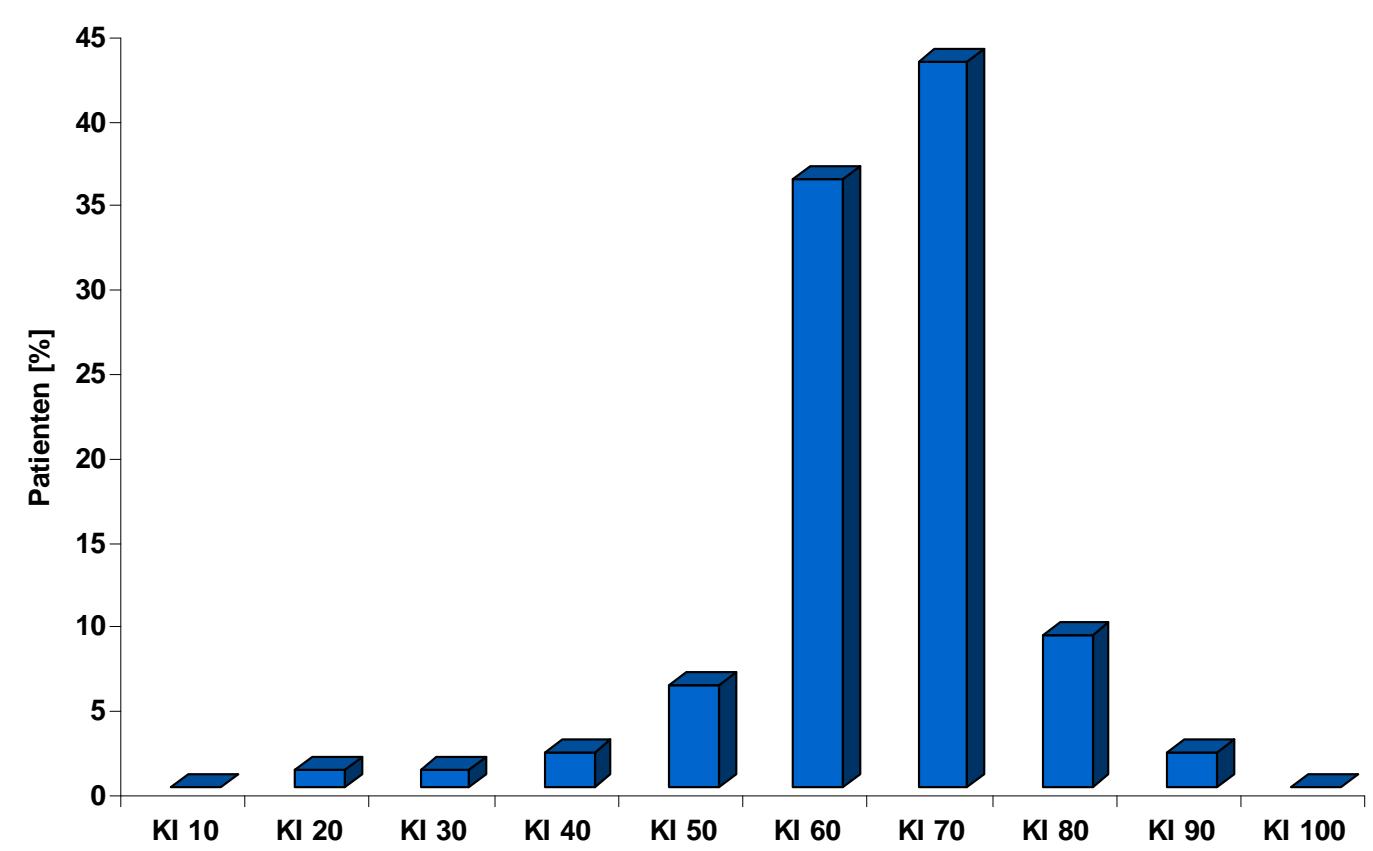




\subsubsection{Resektionsausmaß}

Abhängig von Wachstumscharakteristik und klinischem Zustand der Patienten wurde eine Biopsie (stereotaktisch, offen oder neuronavigiert), eine subtotale oder makroskopisch vollständige Tumorresektion durchgeführt. Hierbei ist zu erwähnen, dass die Differenzierung zwischen subtotaler und makroskopisch vollständiger Resektion auf die subjektive Einschätzung des Operateurs zurückzuführen ist. Eine makroskopisch vollständige Resektion wurde mit $47 \%$ bei dem Großteil des Gesamtkollektivs angegeben, eine Teilresektion bei 36\% durchgeführt, und in rund 17\% der Fälle beschränkte man sich zur Diagnosesicherung auf eine Biopsie (Abb. 16). Im Untersuchungszeitraum ergaben sich keine wesentlichen Verteilungsunterschiede, hinsichtlich des durch die Operateure angegebenen Resektionsausmaßes (Abb.17)

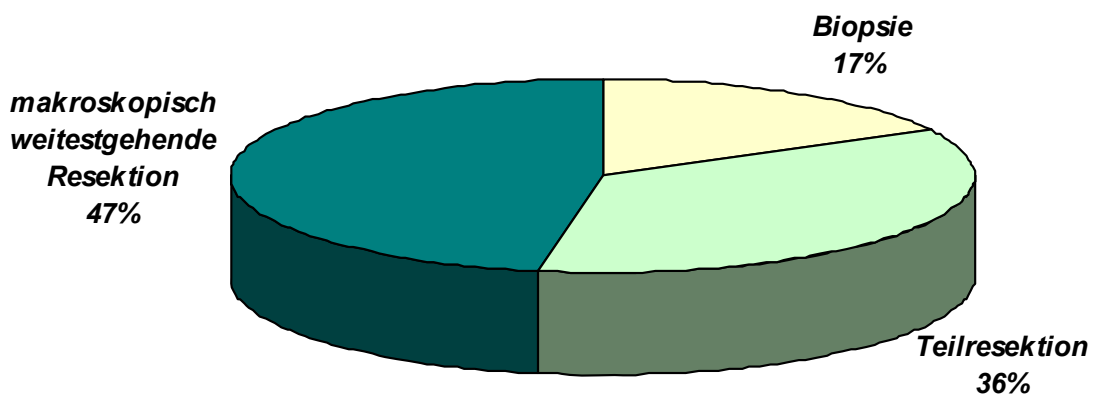

Abb.16: Resektionsausmaß bei Erstoperation (Gesamtkollektiv) 


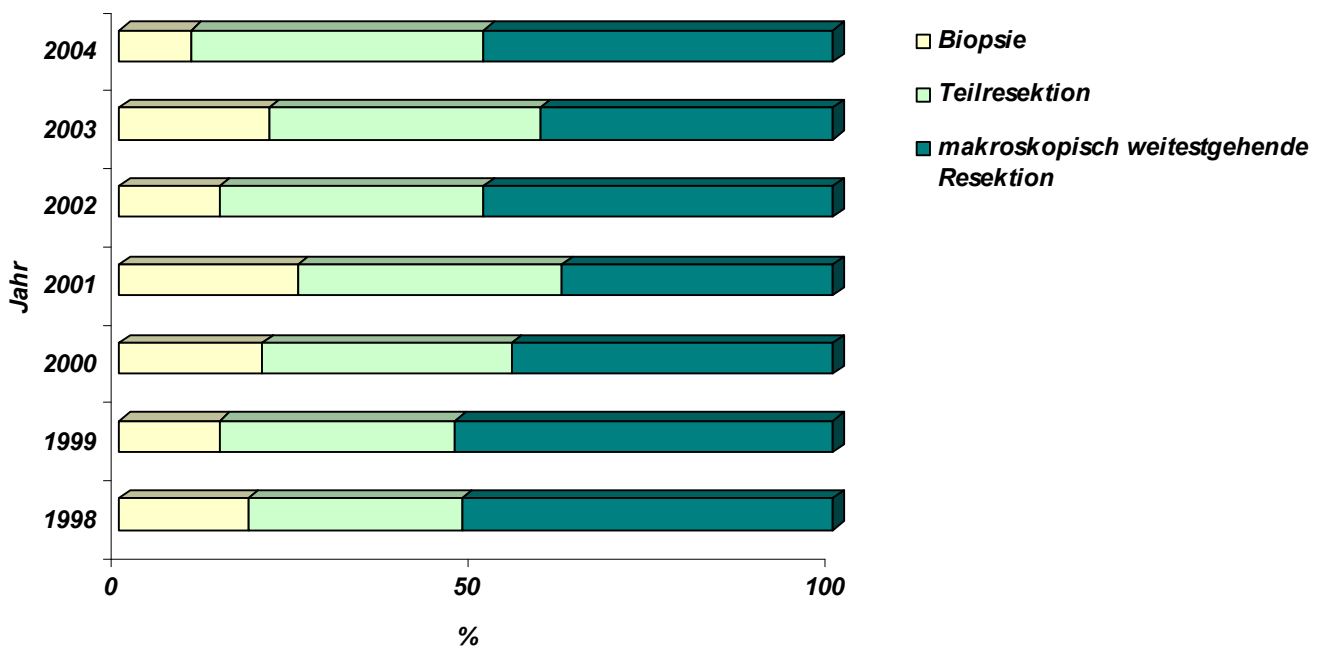

Abb.17: Resektionsausmaß bei Erstoperation in den einzelnen Jahren

\subsubsection{Strahlentherapie}

Der operativen Therapie schloss sich gegebenenfalls die Bestrahlungstherapie an. Insgesamt 28\% aller Patienten erhielten nach Diagnosestellung keine Strahlentherapie. Unter den strahlentherapierten Patienten konnte zwischen der vollständig abgeschlossenen adjuvanten Radiotherapie (fraktioniert 58-60 Gy, Tumorbett mit 2cm Sicherheitsabstand) und der konkomitanten Radiochemotherapie (zeitgleich 75 mg Temozolomid / $\mathrm{m}^{2}$ Körperoberfläche täglich) unterschieden werden. Außerdem zeigte sich, dass ein geringer Anteil der Patienten des Gesamtkollektivs eine adjuvante Bestrahlungstherapie zwar begann, diese jedoch im Verlauf wegen klinischer Verschlechterung abbrechen musste (11\%). Den größten Anteilt mit 54\% bildete die Gruppe der Patienten mit vollständiger adjuvanter Radiatio. Die konkomitante Radiochemotherapie wurde in unserem Kollektiv erstmals im Jahr 2004 durchgeführt und dokumentierte sich anteilsmäßig somit lediglich mit 7\%. 


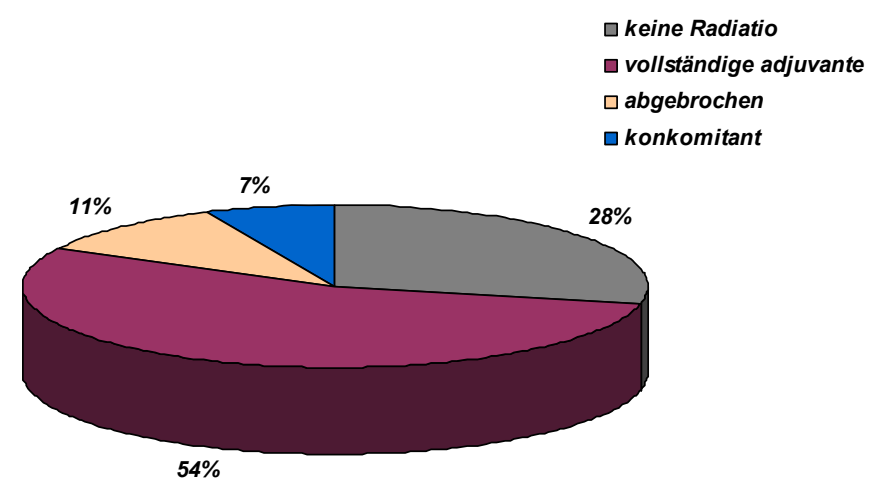

Abb.18: Strahlentherapie nach Diagnosesicherung (Gesamtpopulation)

\subsubsection{Chemotherapie}

Eine Chemotherapie nach Erstoperation (first-line-Chemotherapie) wurde insgesamt bei 43\% aller Patienten des Gesamtkollektivs durchgeführt. Hierbei waren die Therapien mit Temozolomid (150-200mg/m2 KOF) und ACNU (100mg/m² KOF) als Monotherapie nach Bestrahlung mit jeweils 17\% gleichhäufig vertreten. Eine Monotherapie nach dem PCV-Schema wurde nur bei $2 \%$ aller Patienten durchgeführt.

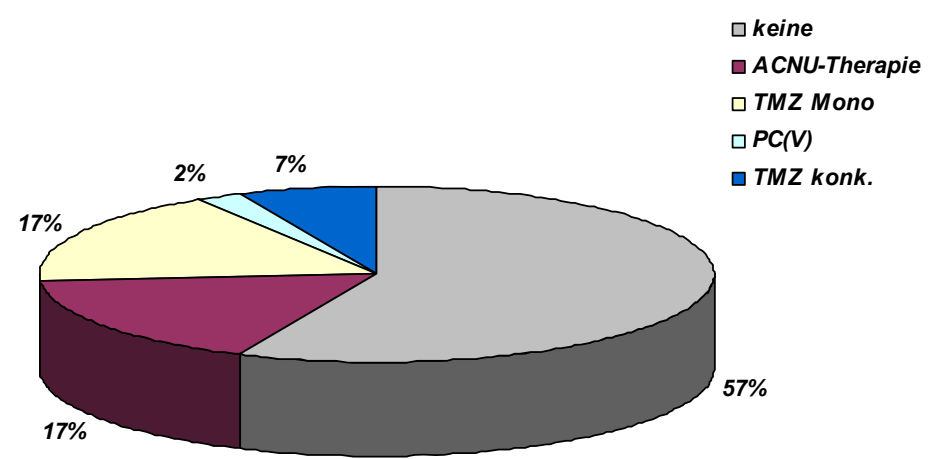

Abb.19: First-line Chemotherapie nach Diagnosesicherung (Gesamtpopulation)

\subsubsection{Gruppierung adjuvanter Therapiemodalitäten}

Letztlich konnten bei der Analyse der adjuvanten Therapiemodalitäten insgesamt 4 Therapiegruppen herausgestellt werden, welche relevante und vergleichbare Patientenzahlen aufwiesen und für die Überlebenszeitanalysen und Progressanalysen herangezogen wurden. Die Chemotherapie als alleinige adjuvante Therapie, also ohne 
Strahlentherapie, wurde bei einer sehr kleinen Patientenzahl durchgeführt, die auf Grund der geringen Fallzahl keine weiterführende Analyse erlaubte.

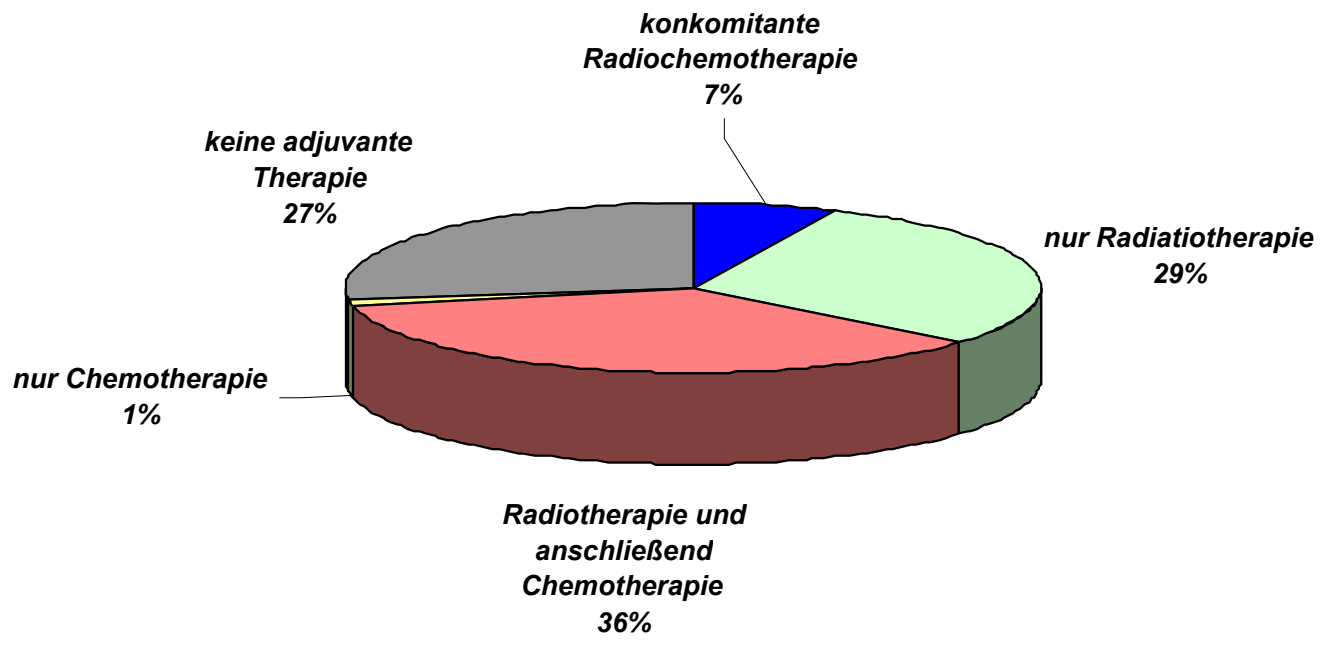

Abb.20: Adjuvante Therapien nach Diagnosesicherung (Gesamtpopulation)

Bei der grafischen Darstellung der adjuvanten Therapien, über den Zeitraum von 1998 bis 2004, dokumentierte sich im letzten Abschnitt des Untersuchungszeitraumes ein Absinken der alleinigen Radiotherapie und der Radiotherapie mit anschließender Chemotherapie, zugunsten der konkomitanten Radiochemotherapie, als Ausdruck der veränderten Therapiestandards.

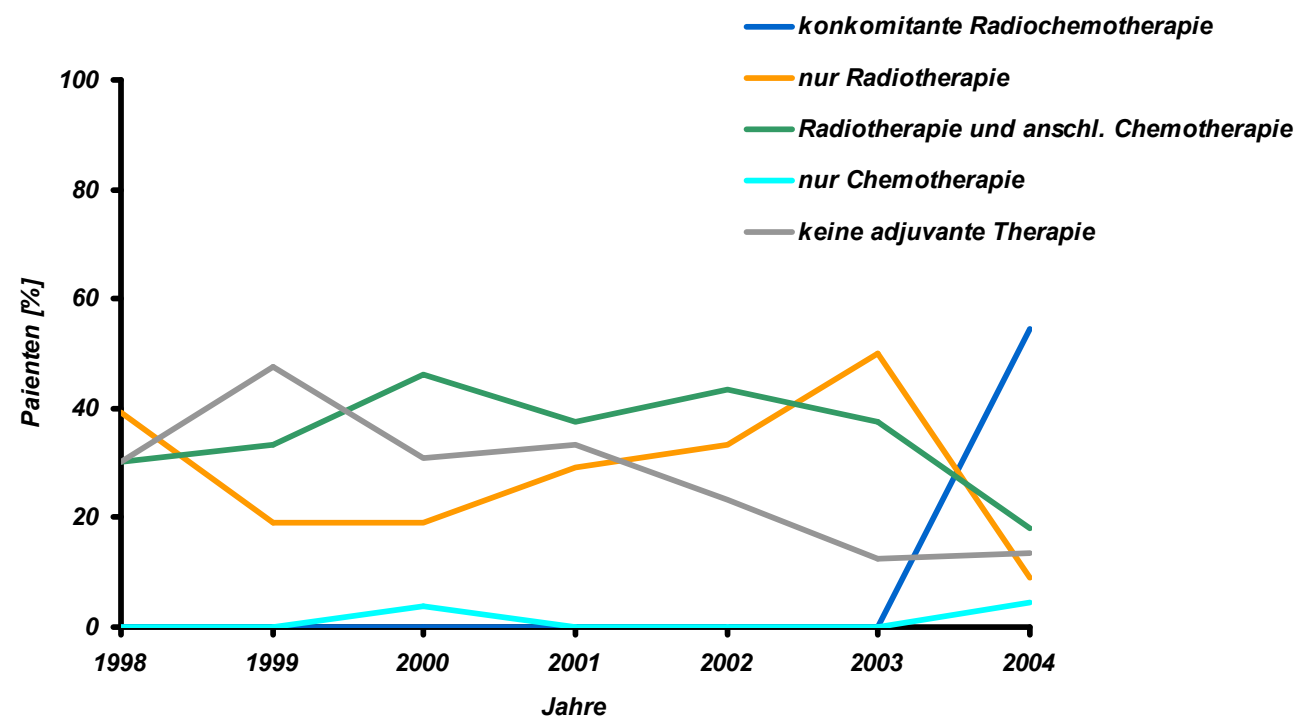

Abb.21: Adjuvante Therapien über den Untersuchungszeitraum (Gesamtpopulation) 


\subsection{8 Überlebenszeiten und progressfreie Intervalle}

Die mediane Überlebenszeit des Gesamtkollektivs betrug 9 Monate, unabhängig von allen prognostischen und therapeutischen Einflussgrößen. 113 Patienten des Gesamtkollektivs überlebten nach Diagnosestellung weniger als 1 Jahr. Zwischen einem und zwei Jahren überlebten 45 Patienten, über 2 Jahre und weniger als drei Jahre überlebten 12 Patienten. 10 Patienten zeigten ein längeres Überleben als drei Jahre.

Die Gruppe der Patienten mit Überlebenszeiten über 36 Monate war mit 10 Patienten jedoch auffallend groß und ließ trotz histologischer Diagnose eines Glioblastoms WHO Grad IV tumorspezifische Besonderheiten vermuten. In die Gruppe der für Glioblastompatienten als Langzeitüberleber bezeichneten Patienten, das sind Patienten, die 24 Monate und länger überlebten, fielen insgesamt 22 Fälle. Diese wurden zu einem späteren Zeitpunkt noch einmal gesondert betrachtet. Die medianen Überlebenszeiten und die klinisch und radiologisch progrssionsfreien Zeiten über die Untersuchungsjahre sind in Abbildung 23 dargestellt.

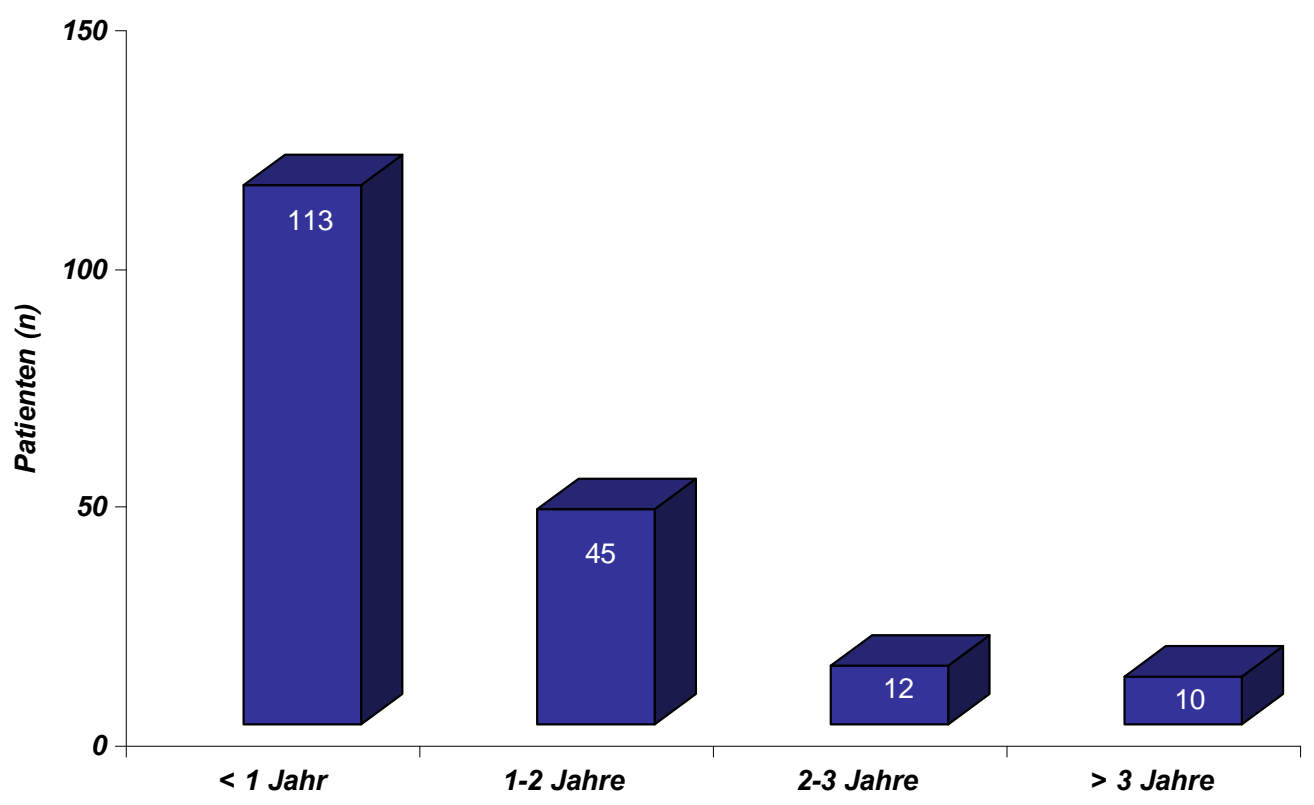

Abb.22: Überlebenszeiten der Gesamtpopulation nach Diagnosestellung unabhängig von prognostischen oder therapeutischen Einflussfaktoren 


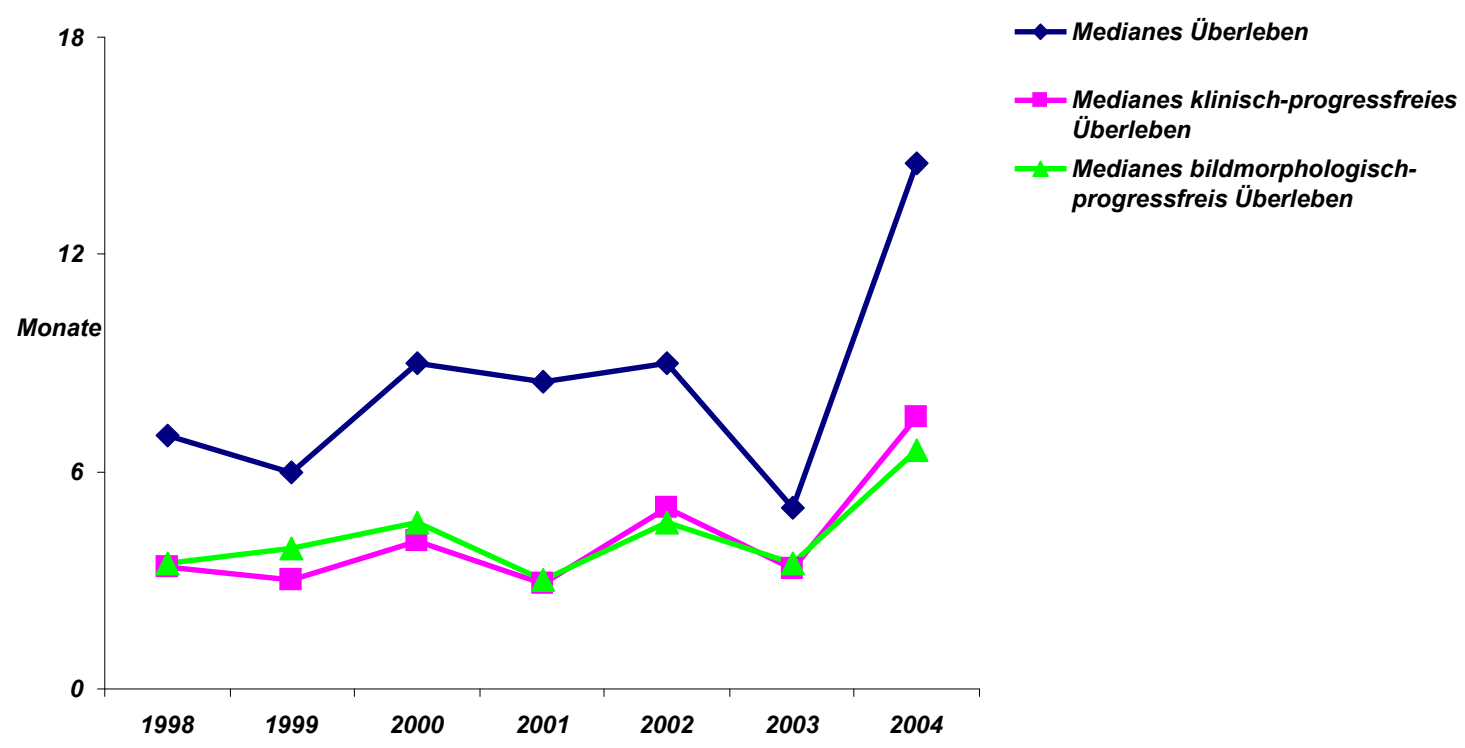

Abb. 23: Medianes Gesamtüberleben, sowie bildmorphologisch- und klinisch-progressfreie Überlebenszeiten , unabhängig von prognostischen oder therapeutischen Einflussfaktoren

\section{2 Überlebenswahrscheinlichkeiten (Survivalanalyse)}

Die Überlebenswahrscheinlichkeiten werden in Form einer Kaplan-Meyer-Kurve dargestellt. Bei der Analyse des Gesamtüberlebens aller untersuchten Patienten fand sich eine 1-Jahres-Überlebenswahrscheinlichkeit von 34\%, die 2-JahresÜberlebenswahrscheinlichkeit lag bei $12 \%$, ungeachtet jeglicher therapeutischer oder prognostischer Einflussgrößen. Im Vergleich der Untersuchungszeiträume vor Einführung der konkomitanten Radiochemotherapie ab 2003 zeigte sich eine Steigerung der 1-Jahres-Überlebenswahrscheinlichkeit von 31\% vor und auf $43 \%$ ab dem Jahr 2003. Hierbei war jedoch zu berücksichtigen, dass die Patientengruppen der einzelnen Jahre nicht homogen waren und eine unterschiedliche Verteilung von wichtigen prognostischen Einflussgrößen, wie z.B. Alter und Karnofsky-Index vorlag. Außerdem waren auch therapeutische Einflussfaktoren vorerst komplett unberücksichtigt geblieben. Für alle Berechnungen der Überlebenswahrscheinlichkeiten wurden daher die zu betrachtenden Untergruppen gesondert nach solchen Einflussfaktoren aufgeschlüsselt und in tabellarischer Form abgebildet (für die unterschiedlichen Untersuchungsjahre siehe Tabelle 3). 


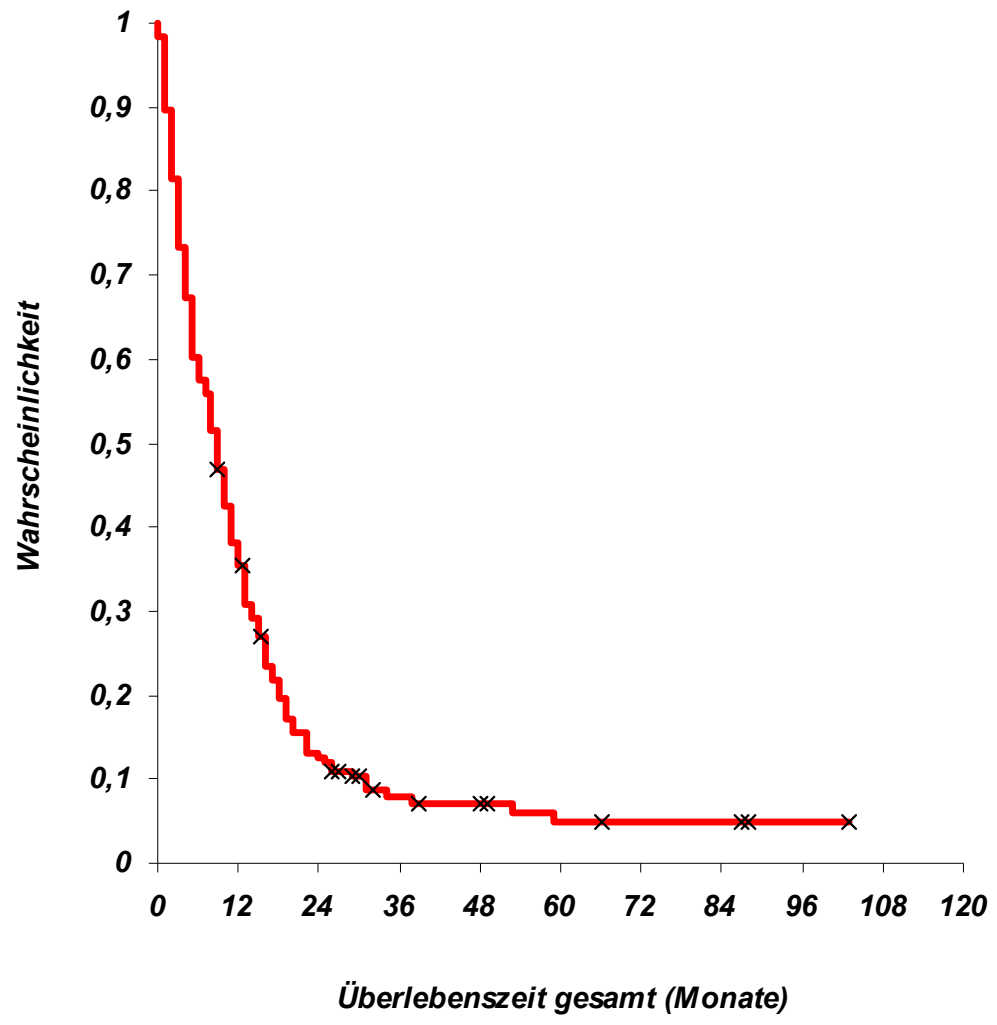

Abb.24: Überlebenswahrscheinlichkeit des Gesamtkollektivs ohne prognostische oder therapeutische Einflussfaktoren

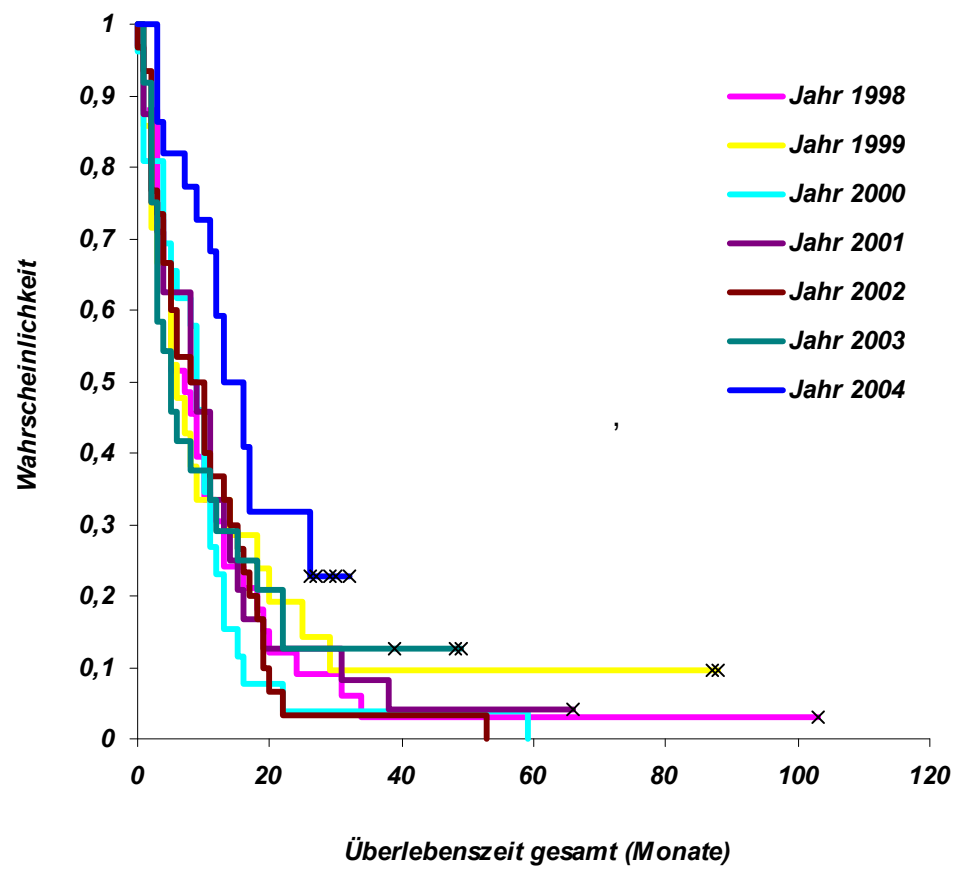

Abb.25: Überlebenswahrscheinlichkeit über die Jahre (Subgruppenaufschlüsselung in Tab. 3), $(P=0,238)$ 


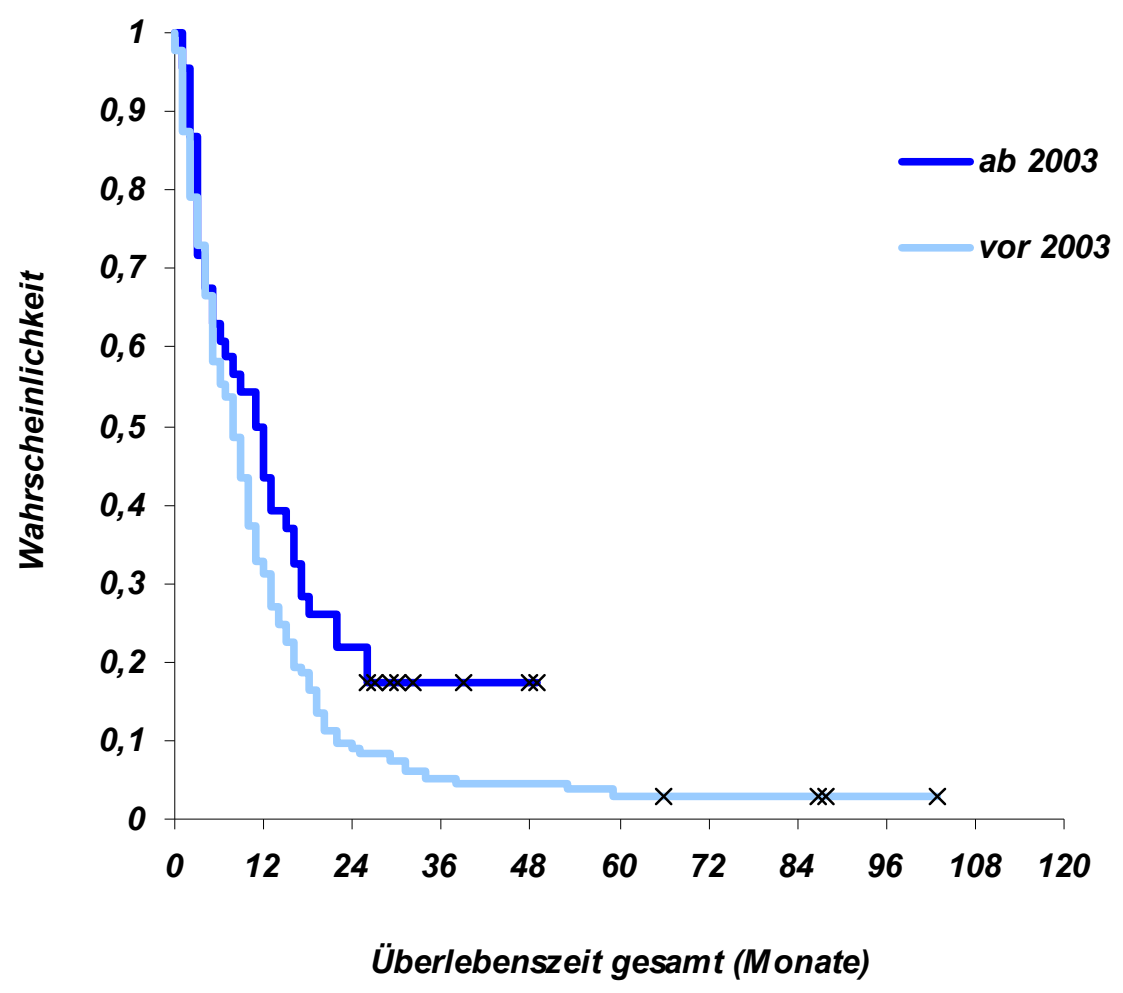

Abb.26: Überlebenswahrscheinlichkeit vor und nach Einführung der konkomitanten Radiochemotherapie im Jahr $2003(P=0,032)$

\subsubsection{Survival in Abhängigkeit vom Alter}

Die Korrelation von Altersklassen der Patienten mit der Überlebenswahrscheinlichkeit zeigte signifikante Unterschiede mit erwartungsgemäß deutlich geringeren Überlebenswahrscheinlichkeiten proportional zum Lebensalter. In der Gruppe der über 70-jährigen lag die 1-Jahres-Überlebenswahrscheinlichkeit bei nur 8\%, bei den 50 bis 70-Jährigen hingegen stieg sie auf 42\%. Die höchsten 1-JahresÜberlebenswahrscheinlichkeiten zeigte die Gruppe der Patienten, die jünger als 50 Jahre waren, sie lag bei 62\%. In der Tabelle 4 wurden die verschiedenen Altersklassen gegenübergestellt und die Verteilung der verschiedenen Einflussgrößen aufgezeigt. Desweiteren ließen sich in der Tabelle das mittlere und mediane klinische und bildmorphologische progressfreie Intervall ablesen. 


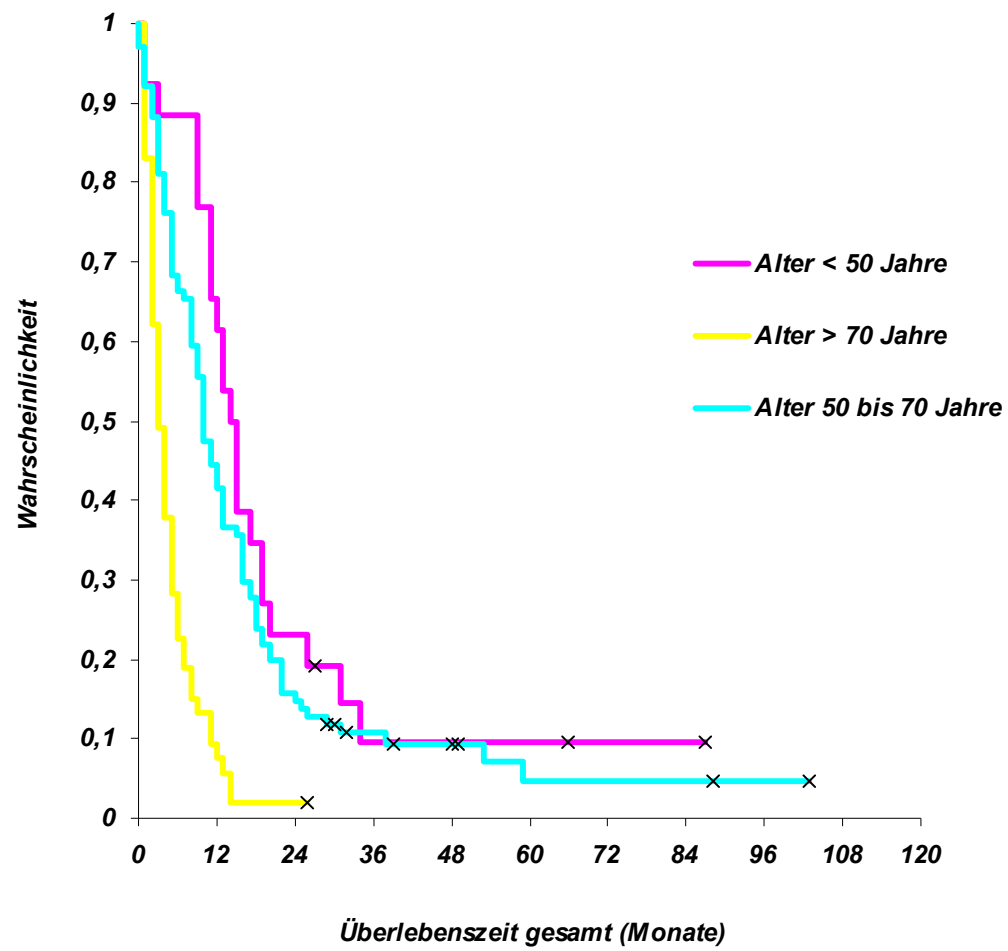

Abb. 27: Überlebenswahrscheinlichkeit der verschiedenen Altersgruppen im Vergleich $(P=<0,01)$

\begin{tabular}{|c|c|c|c|}
\hline Altersgruppen (Jahre) & $>70$ & 50 bis 70 & $<50$ \\
\hline Patienten (n) & 53 & 101 & 26 \\
\hline Biopsie & $23 \%$ & $16 \%$ & $11 \%$ \\
\hline Teilresektion & $42 \%$ & $33 \%$ & $35 \%$ \\
\hline Vollresektion & $35 \%$ & $51 \%$ & $54 \%$ \\
\hline Mittlerer Karnofsky-Index & 60 & 70 & 70 \\
\hline Karnofsky-Index (von-bis) & $30-70$ & $20-90$ & $50-90$ \\
\hline Medianes Alter (Jahre) & 74 & 62 & 43 \\
\hline Keine adjuvante Therapie & $49 \%$ & $20 \%$ & $12 \%$ \\
\hline Nur Strahlentherapie & $32 \%$ & $35 \%$ & $4 \%$ \\
\hline Strahlentherapie und anschl. Chemotherapie & $15 \%$ & $38 \%$ & $72 \%$ \\
\hline Konkomitante Radiochemotherapie & $4 \%$ & $7 \%$ & $12 \%$ \\
\hline \multicolumn{4}{|l|}{ Gesamtüberleben (Monate) } \\
\hline Mittelwert & 4,9 & 14,8 & 19,5 \\
\hline Standardabweichung & 4,6 & 16,5 & 18,9 \\
\hline Median & 3 & 10 & 14,5 \\
\hline \multicolumn{4}{|l|}{ Klinisch-progressfreie Zeit (Monate) } \\
\hline Mittelwert & 3,6 & 7,5 & 7,4 \\
\hline Standardabweichung & 3,1 & 8,9 & 5,2 \\
\hline Median & 2,6 & 4,1 & 6,5 \\
\hline \multicolumn{4}{|l|}{ Bildmorphologisch-progressfreie Zeit (Monate) } \\
\hline Mittelwert & 3,8 & 5,8 & 6,6 \\
\hline Standardabweichung & 3 & 6,6 & 4,5 \\
\hline Median & 3 & 4,1 & 6,2 \\
\hline
\end{tabular}

Tab. 4: Aufschlüsselung der Gesamtpopulation nach Altersgruppen 


\subsubsection{Survival in Abhängigkeit vom präoperativen Karnofsky-Status}

Auch bei der Korrelation des präoperativen Karnofsky-Index (KI) mit der Überlebenswahrscheinlichkeit zeigten sich signifikante Unterschiede zwischen den einzelnen Untergruppen, mit einem Indexwert von unter-, gleich- und über 70 Punkten. Die größte Überlebenswahrscheinlichkeiten erreichte die Patientengruppe mit einem Karnofsky-Index von 80 und mehr Punkten vor der ersten Operation. Hier lag die 1Jahres-Überlebenswahrscheinlichkeit bei 73\%. Die Gruppe mit einem KarnofskyIndex von 70 Punkten (zugleich Mittelwert der Gesamtpopulation) hatte eine 1Jahres-Überlebenswahrscheinlichkeit von $37 \%$ und lag somit deutlich über der 1Jahres-Überlebenswahrscheinlichkeit der Gruppe unter 70 Punkten, diese lag nur bei $22 \%$. Aufgrund der inhomogenen Verteilung von Einflussfaktoren in den einzelnen Untergruppen, wurde in Tabelle 5 auch hier wieder eine Aufschlüsselung der Subgruppen durchgeführt.

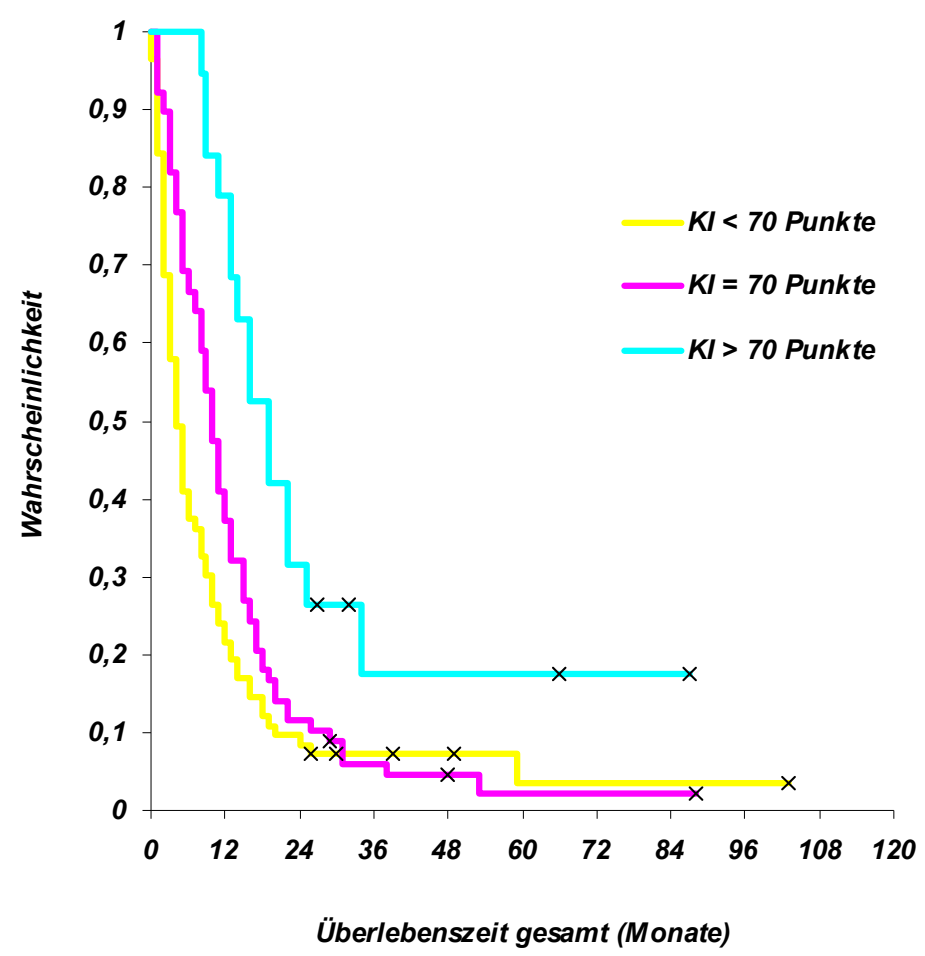

Abb. 27: Survivalanalyse in Abhängigkeit vom Karnofsky-Index präoperativ $(P=<0,01$ 


\begin{tabular}{|c|c|c|c|}
\hline Präoperativer Karnofsky-Index & $<70$ Punkte & 70 Punkte & $>70$ Punkte \\
\hline Patienten $(n)$ & 83 & 78 & 19 \\
\hline Medianes Alter (Jahre) & 68 & 63 & 52 \\
\hline Alter (von-bis) & $30-85$ & $33-84$ & $31-67$ \\
\hline Biopsie & $22 \%$ & $14 \%$ & $10 \%$ \\
\hline Subtotale Resektion & $48 \%$ & $28 \%$ & $16 \%$ \\
\hline Makroskopisch vollständige Resektion & $30 \%$ & $58 \%$ & $74 \%$ \\
\hline Keine adjuvante Therapie & $41 \%$ & $18 \%$ & $5 \%$ \\
\hline Nur Bestrahlung & $38 \%$ & $24 \%$ & $16 \%$ \\
\hline Bestrahlung und anschl. Chemotherapie & $14 \%$ & $52 \%$ & $74 \%$ \\
\hline Konkomitante Radiochemotherapie & $8 \%$ & $6 \%$ & $5 \%$ \\
\hline \multicolumn{4}{|l|}{ Gesamtüberleben (Monate) } \\
\hline Mittelwert & 9,5 & 13 & 24,3 \\
\hline Standardabweichung & 14,8 & 13,3 & 20,1 \\
\hline Median & 4 & 10 & 19 \\
\hline \multicolumn{4}{|l|}{ Klinisch-progressfreie Zeit (Monate) } \\
\hline Mittelwert & 5,3 & 6,8 & 8,4 \\
\hline Standardabweichung & 7,5 & 7,2 & 6,4 \\
\hline Median & 3 & 4,7 & 6,8 \\
\hline \multicolumn{4}{|c|}{ Bildmorphologisch-progressfreie Zeit (Monate) } \\
\hline Mittelwert & 5,3 & 5,1 & 6,3 \\
\hline Standardabweichung & 6,9 & 4,3 & 3,8 \\
\hline Median & 3,3 & 4 & 5,6 \\
\hline
\end{tabular}

Tab. 5: Aufschlüsselung der Gesamtpopulation nach Karnofsky-Index vor Diagnosestellung

\subsubsection{Survival in Abhängigkeit vom Alter und Karnofsky-Status}

Bei dieser Überlebenswahrscheinlichkeitsanalyse betrachteten wir die Gesamtpopulation hinsichtlich der zwei verschiedenen Merkmale, Alter und präoperativer Karnofsky-Status. Verglichen wurden die Patienten unter 70 Jahren und einem KI von mindestens 70 Punkten mit der Gruppe der Patienten ab 70 Jahre und einem KI unter 70 Punkten. Die höchste 1-Jahres-Überlebenswahrscheinlichkeit mit 57\% zeigte sich in der Gruppe der jüngeren Patienten mit einem KI von mindestens 70 Punkten. Bei den Patienten ab 70 Jahren, mit einem KI unter 70 Punkten, lag die 1-JahresÜberlebenswahrscheinlichkeit nur noch bei knapp 10\%. In den beiden Gruppen waren die therapeutischen Einflussfaktoren jedoch wieder unterschiedlich verteilt, siehe hierzu Tabelle 6. 


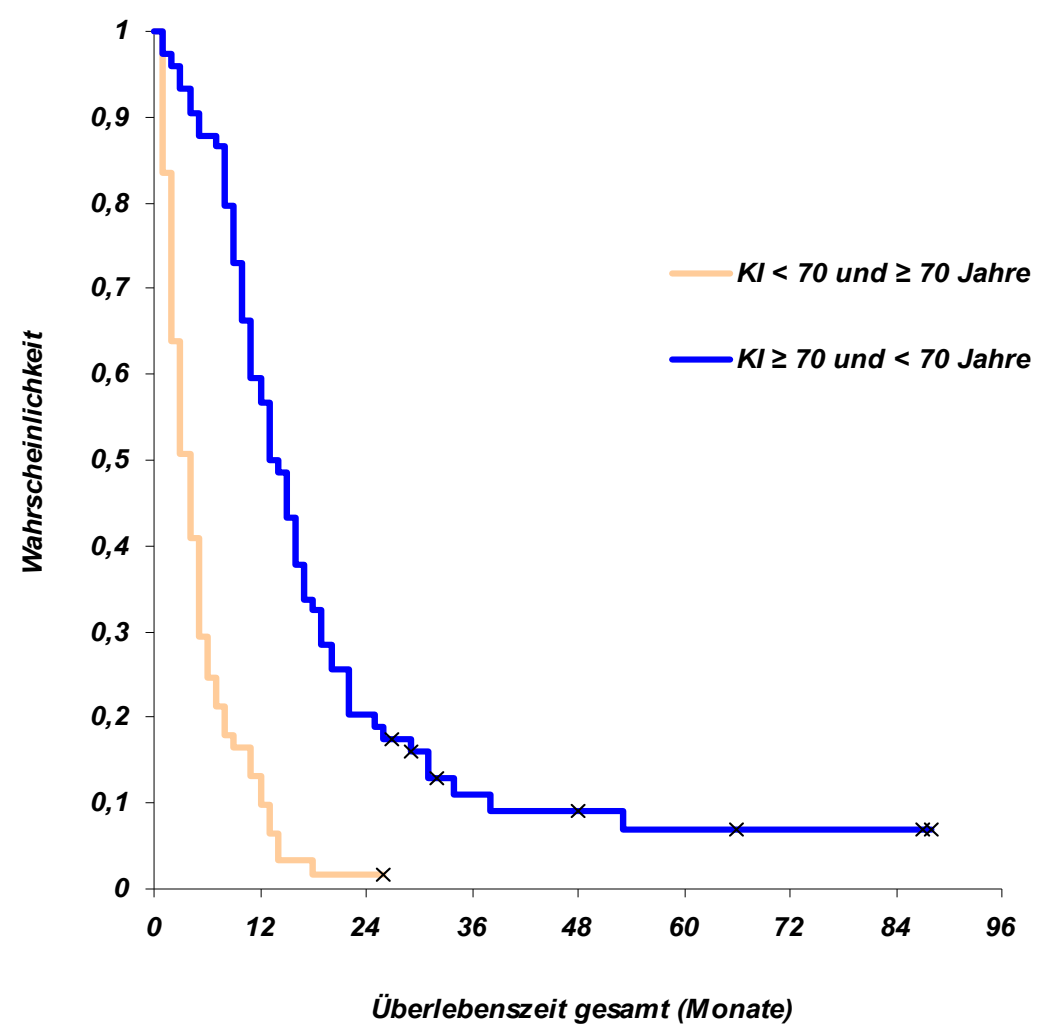

Abb. 28: Überlebenswahrscheinlichkeit korreliert mit Alter und Karnofsky-Index $(P=<0,01)$

\begin{tabular}{|c|c|c|}
\hline Karnofsky-Index kombiniert mit Alter & $K I<70 / \geq 70$ Jahre & $K I \geq 70 /<70$ Jahre \\
\hline Patienten (n) & 61 & 74 \\
\hline Medianes Alter (Jahre) & 74 & 59 \\
\hline Biopsie & $23 \%$ & $9 \%$ \\
\hline Subtotale Resektion & $38 \%$ & $26 \%$ \\
\hline Makroskopisch vollständige Resektion & $39 \%$ & $65 \%$ \\
\hline Keine adjuvante Therapie & $46 \%$ & $9 \%$ \\
\hline Nur Bestrahlung & $36 \%$ & $19 \%$ \\
\hline Bestrahlung und anschl. Chemotherapie & $13 \%$ & $65 \%$ \\
\hline Konkomitante Radiochemotherapie & $5 \%$ & $7 \%$ \\
\hline \multicolumn{3}{|l|}{ Gesamtüberleben (Monate) } \\
\hline Mittelwert & 5,2 & 18,2 \\
\hline Standardabweichung & 4,9 & 16,4 \\
\hline Median & 4 & 13,5 \\
\hline \multicolumn{3}{|l|}{ Klinisch-progressfreie Zeit (Monate) } \\
\hline Mittelwert & 4 & 8,1 \\
\hline Standardabweichung & 3,8 & 7,6 \\
\hline Median & 2,8 & 6,6 \\
\hline \multicolumn{3}{|l|}{ Bildmorphologisch-progressfreie Zeit (Monate) } \\
\hline Mittelwert & 3,8 & 5,9 \\
\hline Standardabweichung & 3,1 & 4,5 \\
\hline Median & 3 & 4,9 \\
\hline
\end{tabular}

Tab. 6: Aufschlüsselung nach den Untergruppen Karnofsky-Index in Kombination mit dem Alter 


\subsubsection{Survival in Abhängigkeit vom Resektionsausmaß}

Auch bei der Korrelation des Resektionsausmaßes der Erstoperation mit der Überlebenswahrscheinlichkeit ergaben sich hochsignifikante Unterschiede. Die 1-JahresÜberlebenswahrscheinlichkeit lag bei den Patienten mit makroskopisch weitestgehender Resektion bei 53\%, bei der Patientengruppe mit subtotaler Tumorresektion hingegen nur noch bei 23 \%. Bei den Patienten die nur eine Biopsie zur Diagnosesicherung erhielten, lag die 1-Jahres-Überlebenswahrscheinlichkeit bei lediglich 11\%. Auch diese drei Subgruppen wurden nach den einzelnen prognostischen und therapeutischen Einflussfaktoren aufgeschlüsselt (siehe Tabelle 7).

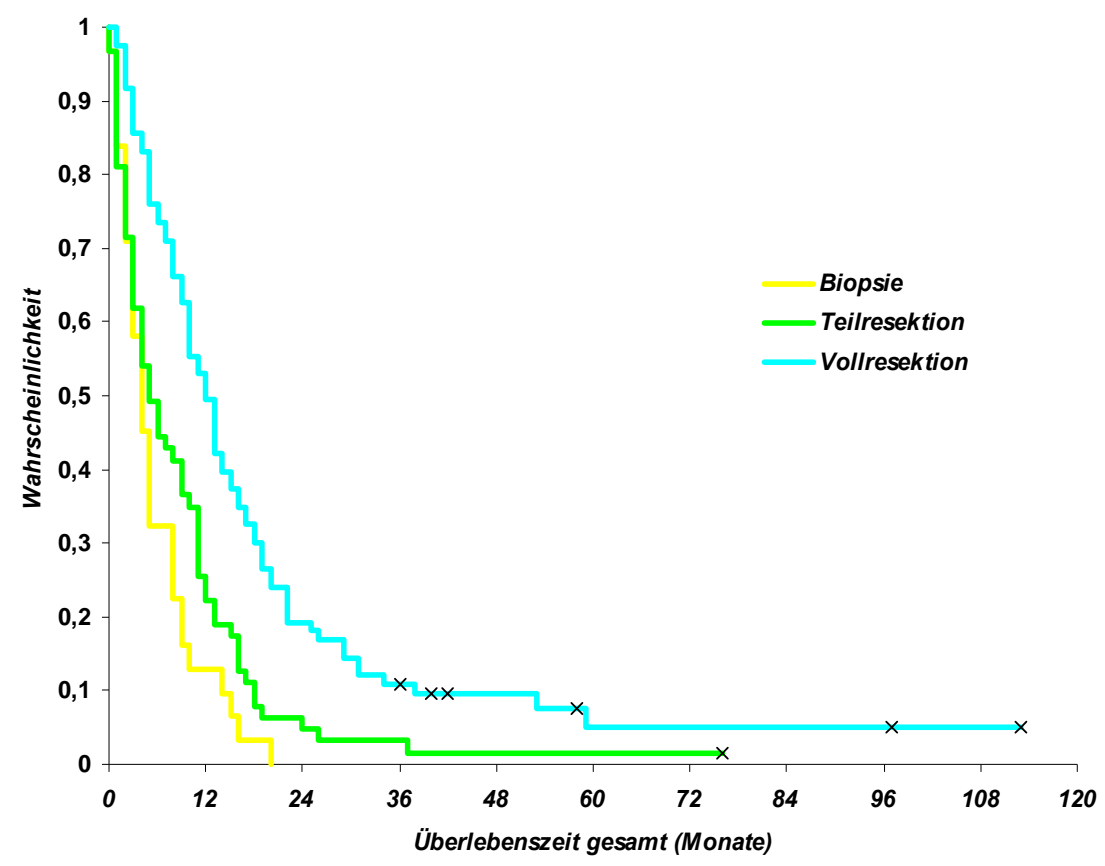

Abb. 29: Überlebenswahrscheinlichkeit in Abhängigkeit vom Resektionsausmaß $(P=<0,01)$ 


\begin{tabular}{|lcccc|}
\hline Resektionsausmaß & Biopsie & $\begin{array}{c}\text { Subtotale Re- } \\
\text { sektion }\end{array}$ & $\begin{array}{c}\text { Makroskopisch } \\
\text { vollständige Resek- } \\
\text { tion }\end{array}$ \\
\hline \hline Patienten (n) & 31 & 64 & 85 \\
\hline Mittlerer Karnofsky-Index & 60 & 60 & 70 \\
Karnofsky-Index (von-bis) & $40-80$ & $20-80$ & $30-90$ \\
Alter von-bis & $41-84$ & $30-85$ & $32-80$ \\
Medianes Alter & 67 & 63 & 63 \\
\hline Keine adjuvante Therapie & $33 \%$ & $42 \%$ & $14 \%$ \\
Nur Bestrahlung & $48 \%$ & $25 \%$ & $27 \%$ \\
Bestrahlung und anschl. Chemotherapie & $13 \%$ & $28 \%$ & $51 \%$ \\
Konkomitante Radiochemotherapie & $6 \%$ & $5 \%$ & $8 \%$ \\
\hline Gesamtüberleben (Monate) & & & \\
Mittelwert & 5,7 & 9 & 17,7 \\
Standardabweichung & 5 & 10,7 & 18,8 \\
Median & $\mathbf{4}$ & $\mathbf{5 , 5}$ & $\mathbf{1 3}$ \\
Klinisch-progressfreie Zeit (Monate) & & & 8,4 \\
Mittelwert & 3,7 & 4,9 & \\
Standardabweichung & 4,4 & 5,3 & 8,9 \\
Median & $\mathbf{2}$ & $\mathbf{3 , 2}$ & $\mathbf{6 , 5}$ \\
Bildmorphologisch-progressfreie Zeit (Monate) & & & 6,6 \\
Mittelwert & 4,4 & 4,1 & 7 \\
Standardabweichung & 4,3 & 3,1 & $\mathbf{5}$ \\
Median & $\mathbf{2 , 9}$ & $\mathbf{3 , 3}$ & \\
\hline
\end{tabular}

Tab. 4: Aufschlüsselung nach Resektionsausmaß

\subsubsection{Survival in Abhängigkeit von der postoperativen, adjuvanten Therapie}

Betrachtete man das postoperative Vorgehen unabhängig vom Resektionsausmaß, Karnofsky-Index und Alter der Patienten, zeigten sich hochsignifikante Unterschiede in der Überlebenswahrscheinlichkeit. Die Patienten, die keine adjuvanten Therapien erhielten, hatten eine 1-Jahres-Überlebenswahrscheinlichkeit von nur 1\%, bei den postoperativ ausschließlich bestrahlten Patienten betrug die 1-JahresÜberlebenswahrscheinlichkeit 20\%. Strahlentherapierte und anschließend zusätzlich chemotherapierte Patienten hatten eine 1-Jahres-Überlebenswahrscheinlichkeit von $61 \%$. In der Gruppe der Patienten mit konkomitant verabreichter Radiochemotherapie mit Temozolomid lag die 1-Jahres-Überlebenswahrscheinlichkeit sogar bei $75 \%$. 


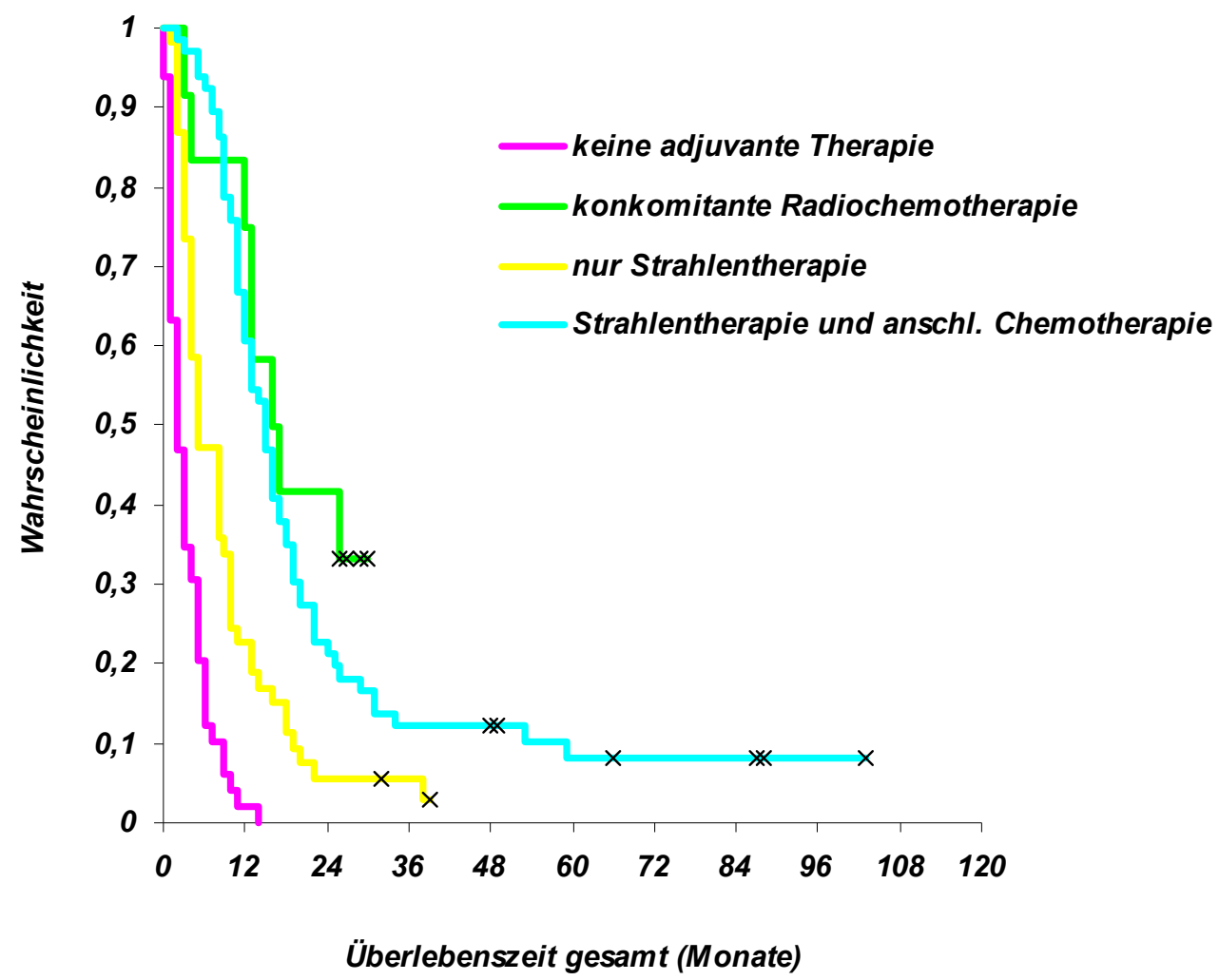

Abb.30: Überlebenswahrscheinlichkeit in Abhängigkeit von der postoperativen Therapie $(P=<0,01)$

Auch als ausschließlich die Patientengruppen miteinander verglichen wurden, die nach operativer Intervention eine adjuvante Therapie erhielten, zeigten sich noch signifikante Unterschiede in den Überlebenswahrscheinlichkeiten zugunsten der zusätzlich zur Strahlentherapie chemotherapierten Patienten. Beim Vergleich der zeitgleich zur Bestrahlung mit Temozolomid (TMZ) chemotherapierten Patienten und derer, die nach Bestrahlung mit ACNU, PCV oder Temozolomid (TMZ) chemotherapiert wurden, dokumentierten sich jedoch keine signifikanten Unterschiede. 


\begin{tabular}{|c|c|c|c|c|}
\hline Postoperative Therapie & keine & $\begin{array}{l}\text { Nur Bestrah- } \\
\quad \text { lung }\end{array}$ & $\begin{array}{l}\text { Bestrahlung und } \\
\text { anschl. Chemo- } \\
\text { therapie }\end{array}$ & $\begin{array}{c}\begin{array}{c}\text { Konkomitante } \\
\text { Radiochemo- } \\
\text { therapie }\end{array} \\
\end{array}$ \\
\hline Patienten ( $n$ ) & 49 & 53 & 64 & 12 \\
\hline Medianer Karnofsky-Index & 60 & 60 & 70 & 70 \\
\hline Karnofsky (von-bis) & $20-80$ & $40-80$ & $30-90$ & $50-90$ \\
\hline Medianes Alter (Jahre) & 71 & 67 & 59 & 59 \\
\hline Alter (von-bis) & $30-84$ & $34-85$ & $31-76$ & $32-73$ \\
\hline Biopsie & $22 \%$ & $17 \%$ & $13 \%$ & $17 \%$ \\
\hline Subtotale Resektion & $35 \%$ & $32 \%$ & $39 \%$ & $25 \%$ \\
\hline Makroskopisch vollständige Resektion & $43 \%$ & $51 \%$ & $48 \%$ & $58 \%$ \\
\hline \multicolumn{5}{|l|}{ Gesamtüberleben (Monate) } \\
\hline Mittelwert & 3,4 & 9 & 21,3 & 18 \\
\hline Standardabweichung & 3,1 & 8,6 & 20,3 & 9,5 \\
\hline Median & 2 & 5 & 15 & 16,6 \\
\hline \multicolumn{5}{|l|}{ Klinisch-progressfreie Zeit (Monate) } \\
\hline Mittelwert & 2,4 & 5,9 & 9,5 & 7,4 \\
\hline Standardabweichung & 2,7 & 6,7 & 9 & 5 \\
\hline Median & 1,4 & 3,3 & 7,1 & 6,4 \\
\hline \multicolumn{5}{|c|}{ Bildmorphologisch-progressfreie Zeit (Monate) } \\
\hline Mittelwert & 2,7 & 5 & 7,3 & 7 \\
\hline Standardabweichung & 2,7 & 4,1 & 7,2 & 4,4 \\
\hline Median & 1,8 & 3,9 & 5,7 & 5,8 \\
\hline
\end{tabular}

Tabelle 5: Aufschlüsselung der Subgruppe postoperative Therapie

Bei der Aufschlüsselung der Gruppen nach adjuvanter Therapiemodalität zeigte sich, dass bei Patienten ohne adjuvante Therapie der höchste Prozentsatz an lediglich biopsierten Patienten zu finden war, und auch das mediane Alter hier am höchsten war. Im Gegensatz dazu waren die umfangreich adjuvant therapierten Patienten deutlich jünger, hatten einen höheren Karnofsky-Index und waren zudem großzügiger operiert worden. Um trotz dieser ungleichen Subgruppenverteilung eine Aussage über die Effektivität der adjuvanten Therapie machen zu können, wurde deshalb die Survivalanalyse diesbezüglich für die Biopsierten, Teilresezierten und Vollresezierten nochmals getrennt durchgeführt. 


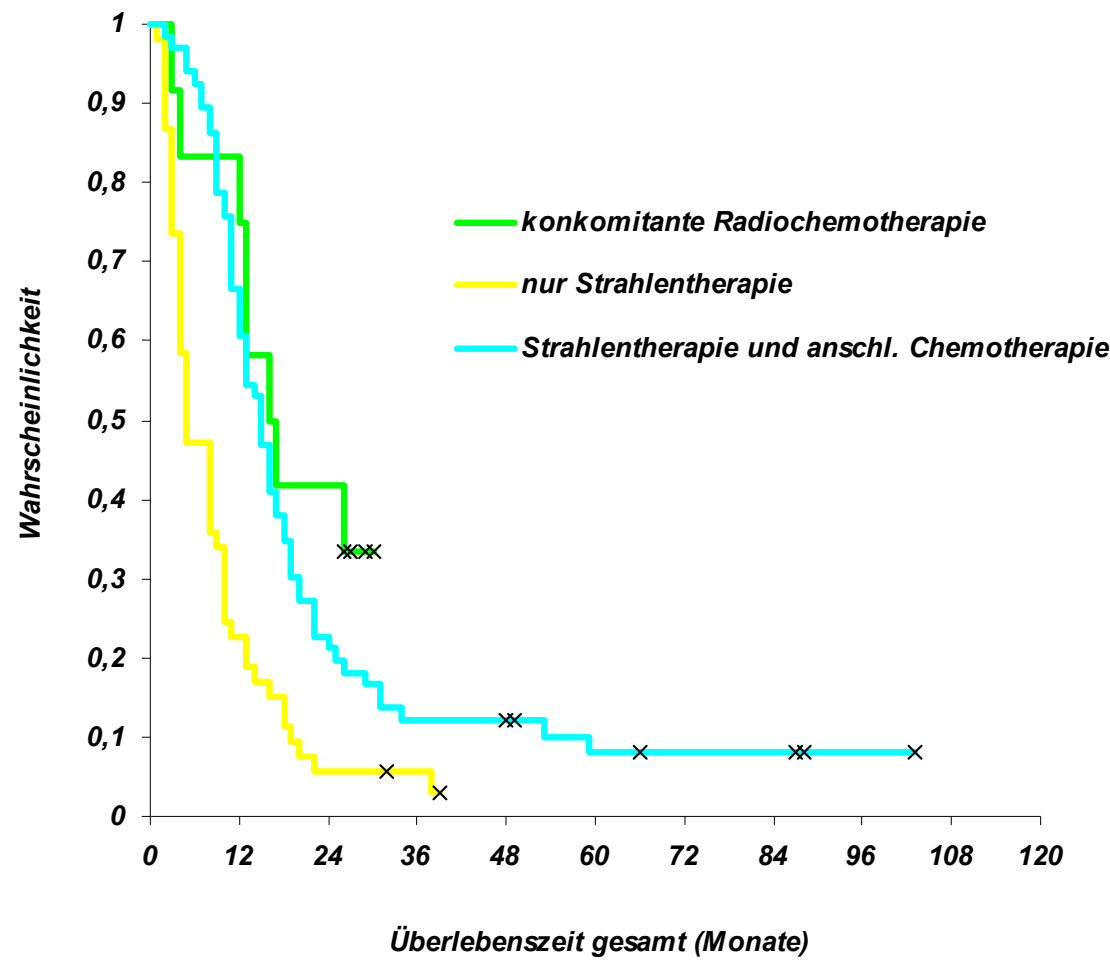

Abb.31: Überlebenswahrscheinlichkeit in Abhängigkeit der adjuvanten Therapiemodalität $(P=<0,01)$

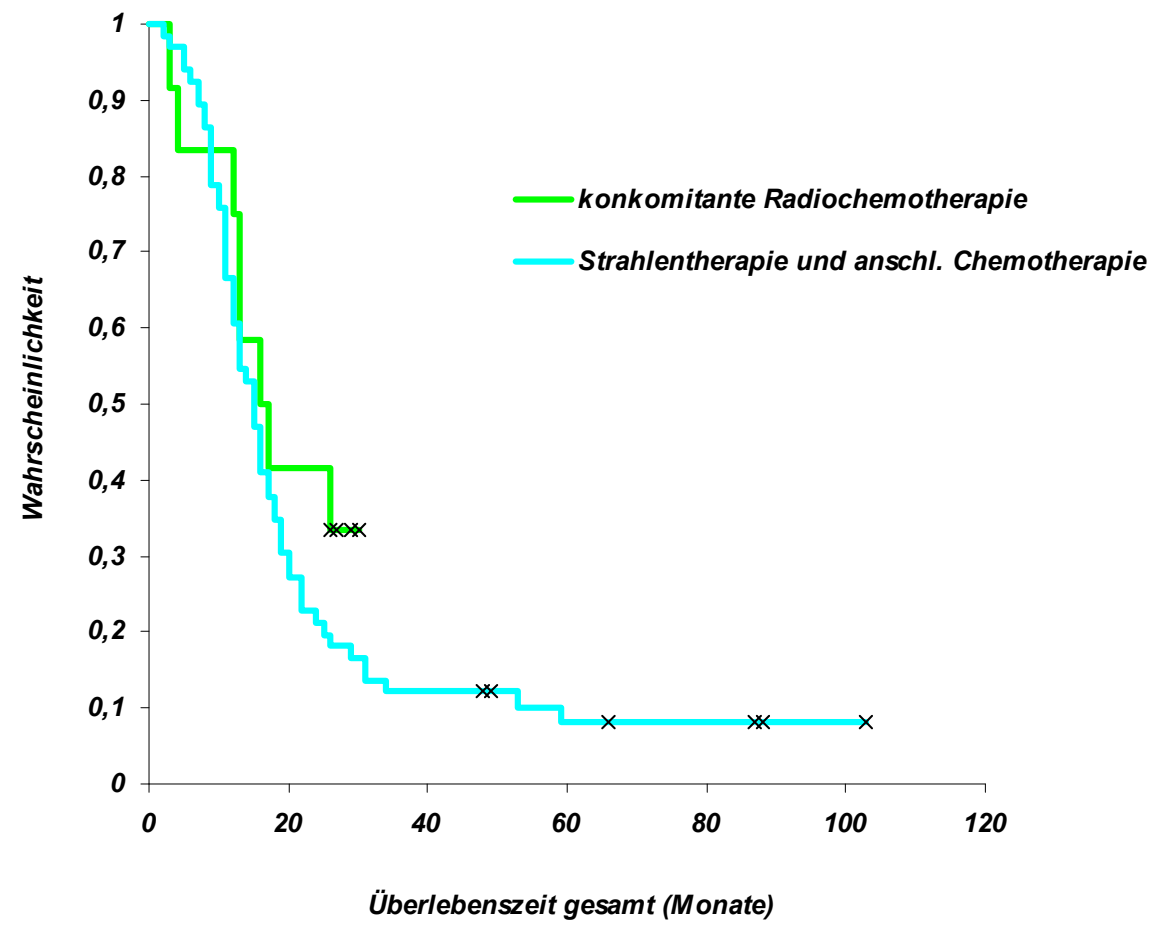

Abb.31: Überlebenswahrscheinlichkeit in Abhängigkeit von Bestrahlungs-begleitender und anschlieBender Chemotherapie $(P=0,29)$ 
3.2.6 Survival in Abhängigkeit von Resektionsausmaß kombiniert mit adjuvanter Therapie

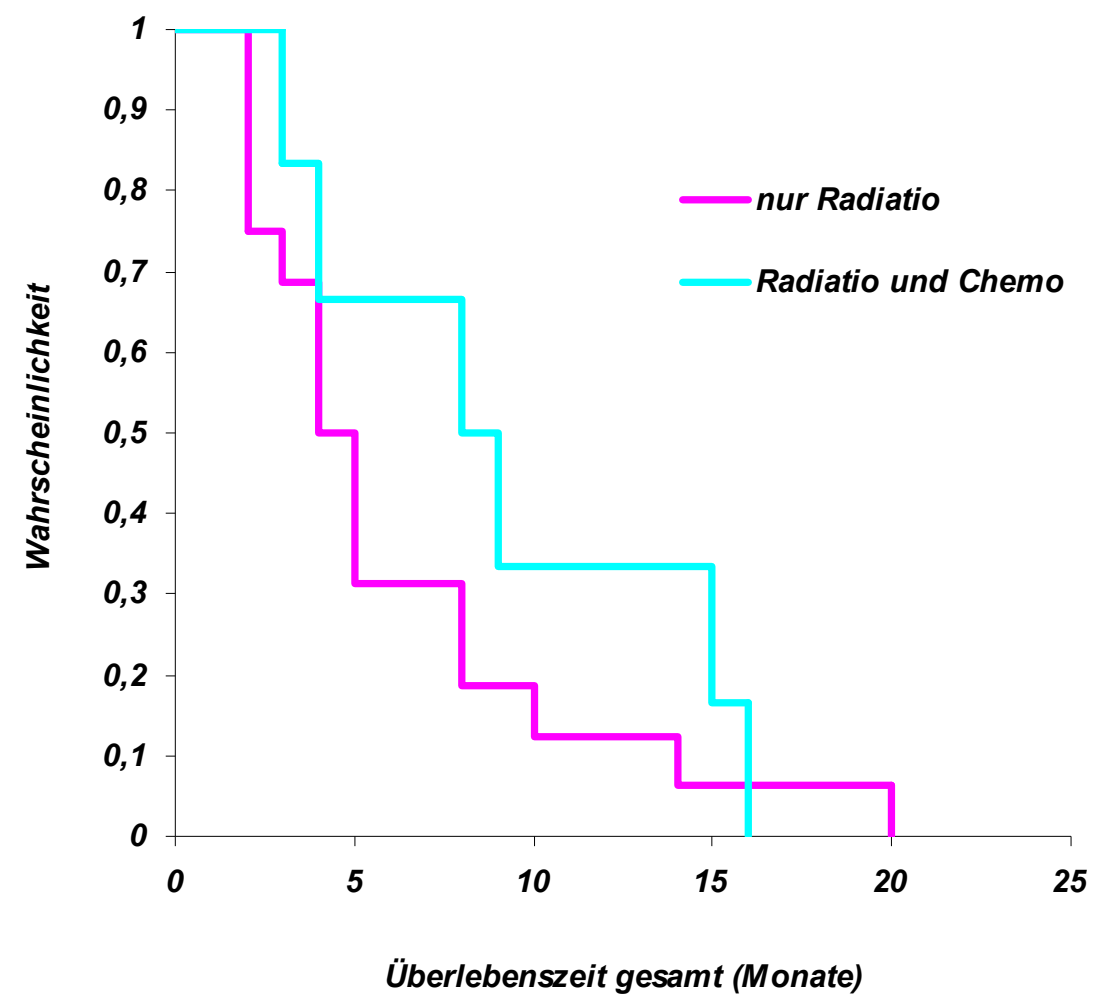

Abb. 32: Survivalanalyse aller Patienten mit Biopsie nach adjuvanter Therapie $(P=0,346)$

Bei den lediglich biopsierten Patienten konnten die Gruppe der zusätzlich bestrahlten und die der bestrahlten und im Anschluss chemotherapierten Patienten miteinander verglichen werden. Hier konnten keine signifikanten Unterschiede in der Überlebenswahrscheinlichkeit festgestellt werden. Bei den subtotal resezierten Patienten hingegen, waren die Ergebnisse signifikant unterschiedlich. Die höchste 1Jahresüberlebenswahrscheinlichkeit mit über $60 \%$ ergab sich für die Gruppe der konkomitant radiochemotherapierten Patienten. Ebenso signifikant waren die Ergebnisse in der Gruppe der makroskopisch vollständig resezierten Patienten. Hier zeigte sich eine 1-Jahres-Überlebenswahrscheinlichkeit für die konkomitant radiochemotherapierten Patienten von $86 \%$. 


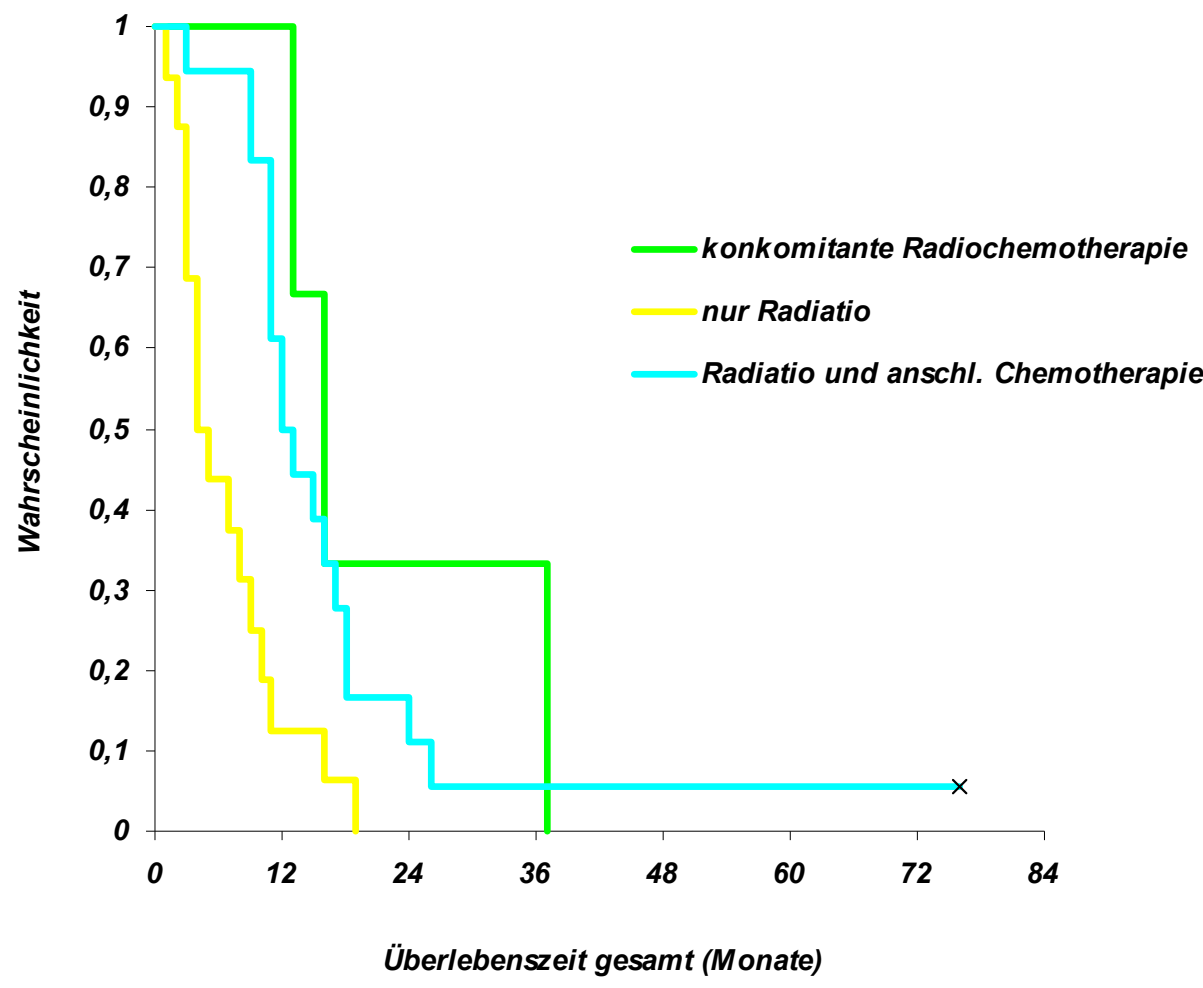

Abb. 33: Survivalanalyse aller Patienten mit Teilresektion nach adjuvanter Therapie $(P=<0,01)$

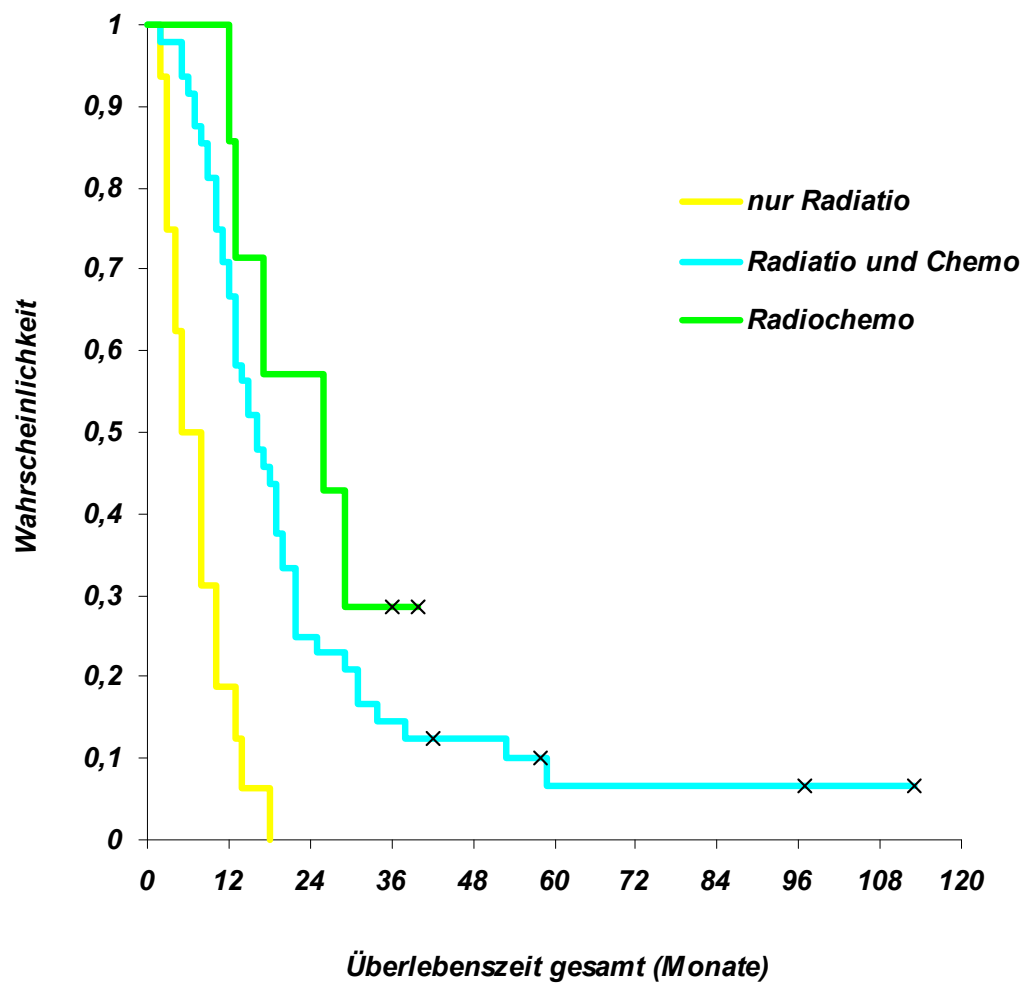

Abb. 34: Survivalanalyse aller Patienten mit Vollresektion nach adjuvanter Therapie $(P=<0,01)$ 
Bei der getrennten Betrachtung der Gruppen mit unterschiedlichem Resektionsausmaß zeigten sich, hinsichtlich der adjuvanten Therapie, signifikante Unterschiede zwischen den subtotal resezierten Patienten und den makroskopisch vollständig resezierten Patienten. In der Gruppe der lediglich biopsierten Patienten waren die Überlebenswahrscheinlichkeiten jedoch nicht mehr signifikant unterschiedlich $(P=0,346)$.

\subsubsection{Survival in Abhängigkeit vom Zytostatikum}

Betrachtet wurden die verschiedenen Chemotherapie-Modalitäten. Auffallend war die starke Repräsentation der Gruppe von Patienten, die keine Chemotherapie erhielten. In dieser Gruppe war die 1-Jahres-Überlebenswahrscheinlichkeit 1 \%. Nur 17 Patienten erhielten eine ausschließliche Chemotherapie mit ACNU, in dieser Gruppe ließ sich eine 1-Jahres Überlebenswahrscheinlichkeit von $27 \%$ berechnen. Bei den übrigen Patienten kam Temozolomid entweder als Monotherapie, als konkomitante Therapie oder als Zweittherapie nach ACNU oder PCV zur Verwendung. Die Patientengruppe, die in erster Linie PCV erhielt, war mit einer Anzahl von 4 Patienten zu klein, um Überlebenswahrscheinlichkeiten zu berechnen. Bei den Patienten, die eine konkomitante Chemotherapie mit Temozolomid erhielten, und denen, die in erster Linie mit ACNU und dann mit Temozolomid behandelt wurden, betrug die 1-JahresÜberlebenswahrscheinlichkeit jeweils 75\%. Die Monotherapie mit Temozolomid erbrachte eine 1-Jahres Überlebenswahrscheinlichkeit von $68 \%$. 


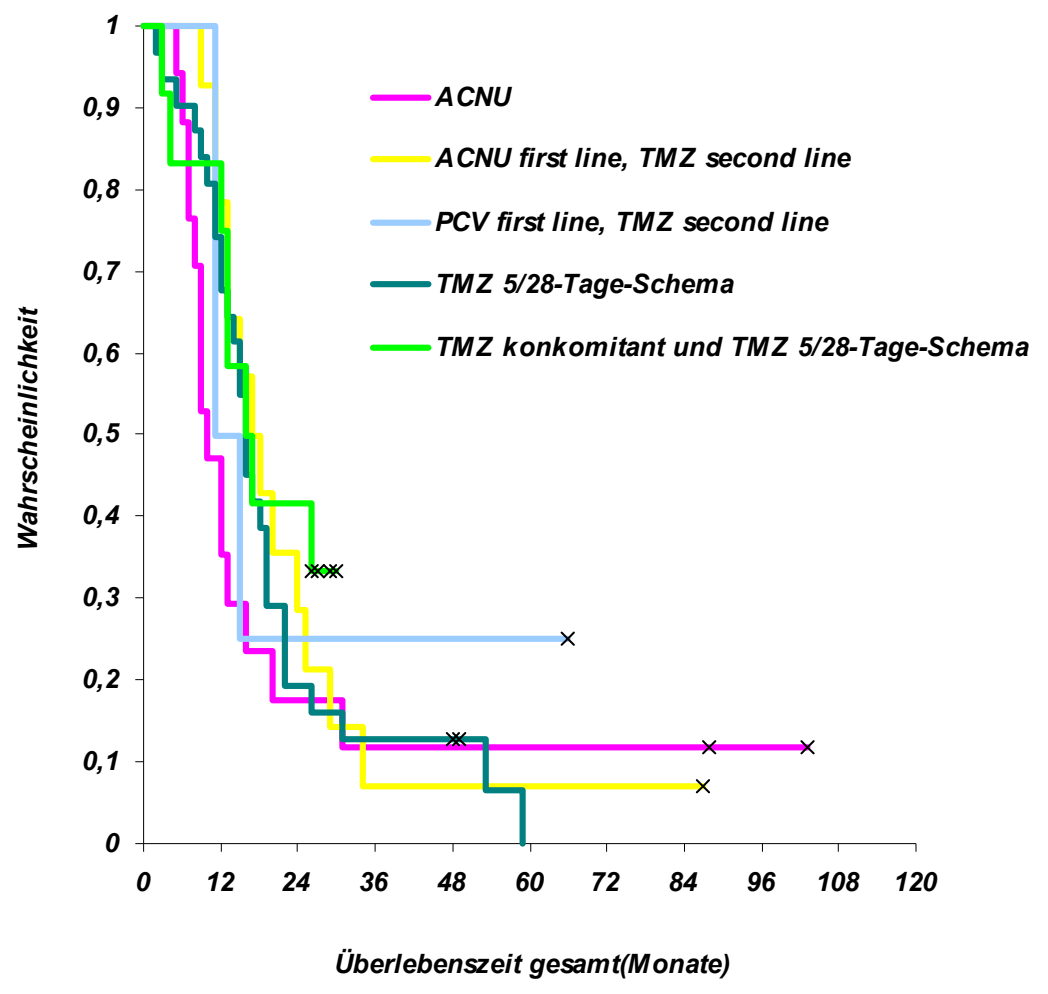

Abb. 35: Korrelation Überlebenswahrscheinlichkeit mit Zytostatikum $(P=0,618)$

\begin{tabular}{|c|c|c|c|c|c|}
\hline Zytostatika & $\begin{array}{l}\text { PCV } \\
\text { ITMZ }\end{array}$ & TMZ & $\begin{array}{l}\text { konk. } \\
+ \text { +TMZ }\end{array}$ & $\begin{array}{l}\text { ACNU } \\
\text { ITMZ }\end{array}$ & ACNU \\
\hline Patienten (n) & 4 & 31 & 12 & 14 & 17 \\
\hline Medianes Alter (Jahre) & 40 & 60 & 59 & 50 & 63 \\
\hline Biopsie & $0 \%$ & $7 \%$ & $17 \%$ & $0 \%$ & $12 \%$ \\
\hline Subtotale Resektion & $25 \%$ & $27 \%$ & $25 \%$ & $29 \%$ & $18 \%$ \\
\hline Makroskopische Vollresektion & $75 \%$ & $64 \%$ & $58 \%$ & $71 \%$ & $70 \%$ \\
\hline Medianer Karnofsky-Index & 70 & 70 & 70 & 70 & 70 \\
\hline Mediane Zykluszahl & $2 / 2$ & 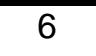 & $1 / 5$ & $3 / 3$ & 1 \\
\hline Re-Operation & $25 \%$ & $42 \%$ & $33 \%$ & $64 \%$ & $35 \%$ \\
\hline \multicolumn{6}{|l|}{ Gesamtüberleben (Monate) } \\
\hline Mitte/wert & 25,8 & 19,7 & 18 & 23,3 & 21,5 \\
\hline Standardabweichung & 26,9 & 14,2 & 9,5 & 19,8 & 28,7 \\
\hline Median & 13 & 16 & 16,5 & 17,5 & 10 \\
\hline \multicolumn{6}{|c|}{ Klinisch-progressfreie Zeit (Monate) } \\
\hline Mittelwert & 7,7 & 11,3 & 7,4 & 11,5 & 4,4 \\
\hline Standardabweichung & 5,4 & 10,6 & 5 & 8,4 & 3 \\
\hline Median & 6,9 & 8,2 & 6,4 & 8,8 & 3,8 \\
\hline \multicolumn{6}{|c|}{ Bildmorphologisch-progressfreie Zeit (Monate) } \\
\hline Mittelwert & 7,4 & 7,9 & 7 & 6,7 & 6,7 \\
\hline Standardabweichung & 5,5 & 8,9 & 4,4 & 3,6 & 6,7 \\
\hline Median & 5,9 & 6,5 & 5,8 & 5,8 & 4,2 \\
\hline
\end{tabular}

Tab. 6: Aufschlüsselung der Chemotherapiegruppen 
Der Vergleich der adjuvanten first-line-Therapie mit ACNU nach Strahlentherapie mit Temozolomid als Monotherapie, im Anschluss an die Strahlentherapie, ergab keine signifikanten Unterschiede bezüglich der Überlebenswahrscheinlichkeiten. Auch bei dem Vergleich der Temozolomid-Monotherapie nach Radiatio und der konkomitanten Therapie mit Temozolomid zeigte sich kein signifikanter Unterschied bezüglich der Überlebenswahrscheinlichkeit

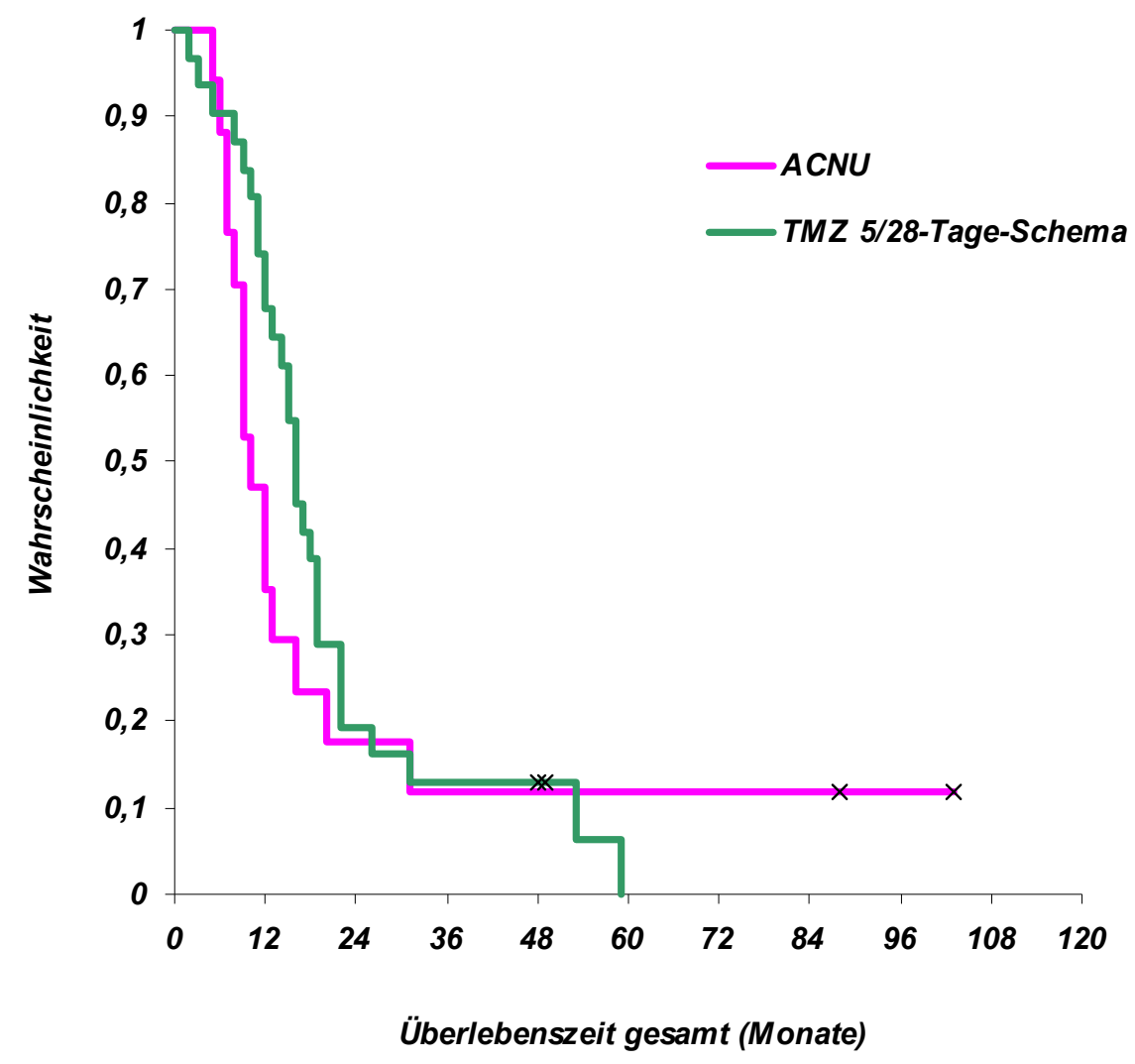

Abb.36: Überlebenswahrscheinlichkeite in Abhängigkeit von Temozolomid- oder ACNU-Monotherapie $(P=0,463)$ 


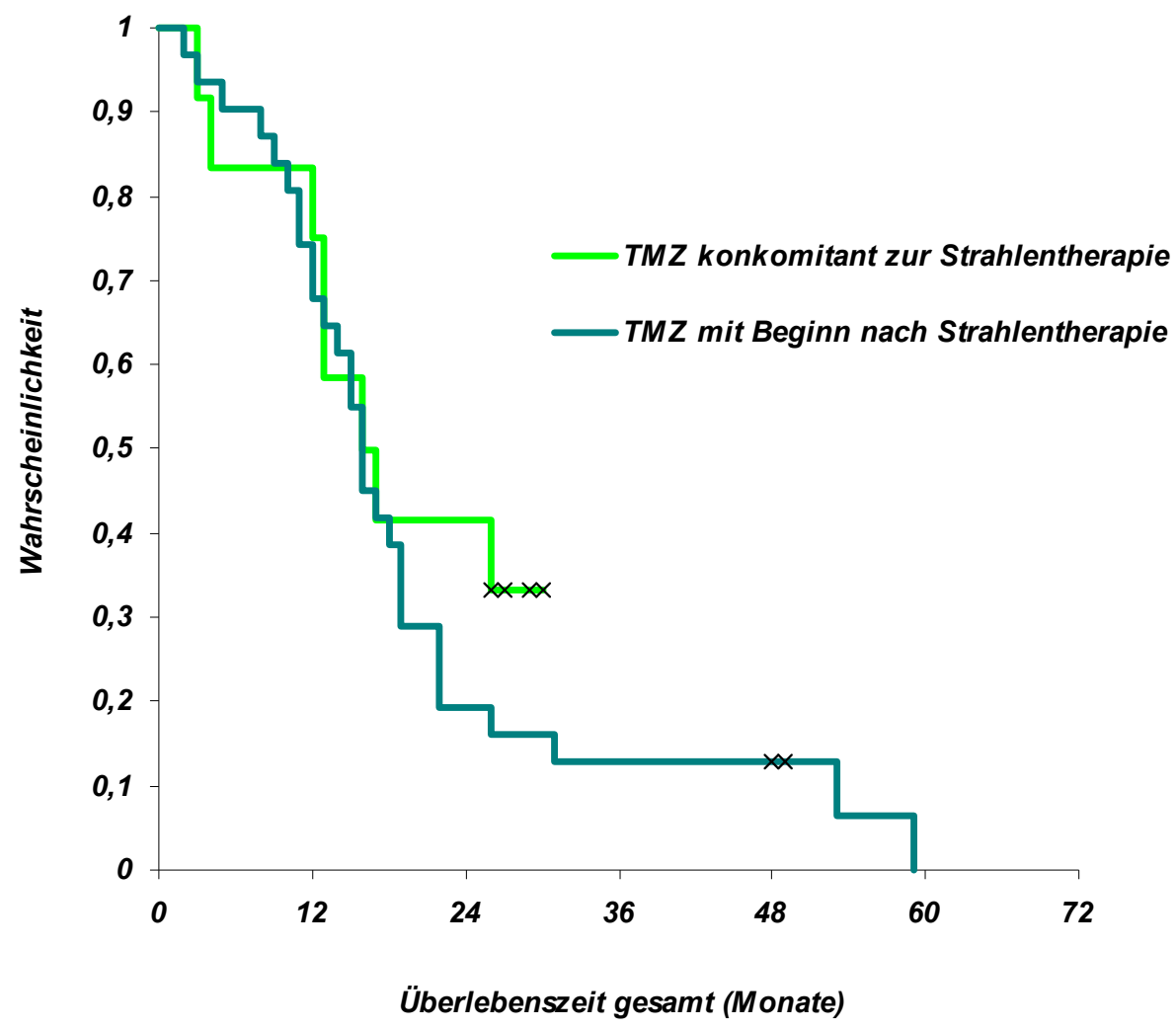

Abb.34: Überlebenswahrscheinlichkeit mit konkomitanter Radiochemotherapie und TemozolomidMonotherapie im Vergleich zur Monotherapie mit Temozolomid nach Bestrahlung $(P=0,381)$

\subsection{Progressanalyse klinisch und bildmorphologisch}

Die mediane klinisch-progressfreie Zeit lag im Gesamtkollektiv bei 4 Monaten, ebenso die bildmorphologisch-progressfreie Zeit nach erster operativer Intervention. Hierbei waren aber vorerst weder prognostische noch therapeutische Einflussfaktoren berücksichtigt. Weder in den einzelnen Jahrgängen untereinander noch beim Vergleich der Patientengruppen, vor und nach Einführung der konkomitanten Radiochemotherapie im Jahre 2003, waren signifikante Unterschiede in der Wahrscheinlichkeit für das Auftreten eines radiologischen oder klinischen Progresses nachzuweisen.

\subsubsection{Progressionsanalyse in Abhängigkeit von Alter und Karnofsky-Index}

Bei dem Einflussfaktor 'Alter' zeigten sich sowohl für die klinische als auch für die bildmorphologische Progressionsanalyse signifikante Ergebnisse. Hinsichtlich des präoperativen Karnofsky-Status waren die Ergebnisse nicht signifikant, wohl aber für beide Faktoren in Kombination. So dokumentierte sich die Wahrscheinlichkeit für ein längeres progressfreies Intervall deutlich höher für Patienten unter 70 Jahren und mit 
einem initialen Karnofsky-Index von mindestens 70 Punkten. Das mediane klinisch progressfreie Intervall lag für diese Patienten bei 6,6 Monaten und das bildmorphologisch-progressfreie Intervall bei 4,9 Monaten. Im Vergleich hierzu traten die Progresse bei Patienten ab 70 Jahren, mit einem Karnofsky-Index von weniger als 70 Punkten, im Median schon nach 2,8 Monaten klinisch und nach 3 Monaten bildmorphologisch auf.

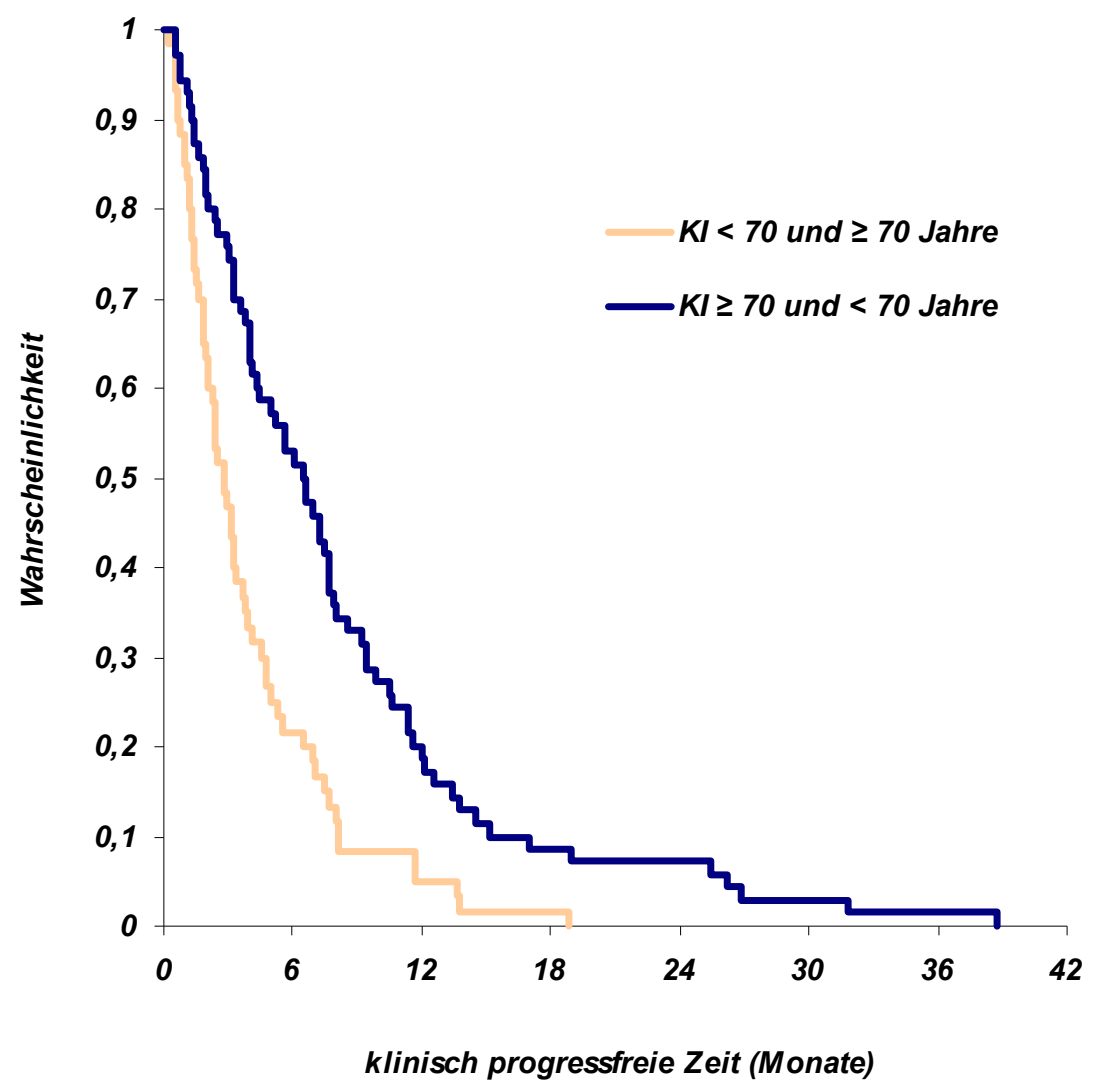

Abb.35: Progressionsanalyse für klinischen Progress korreliert mit Alter und präoperativem KI $(P=<0,01)$ 


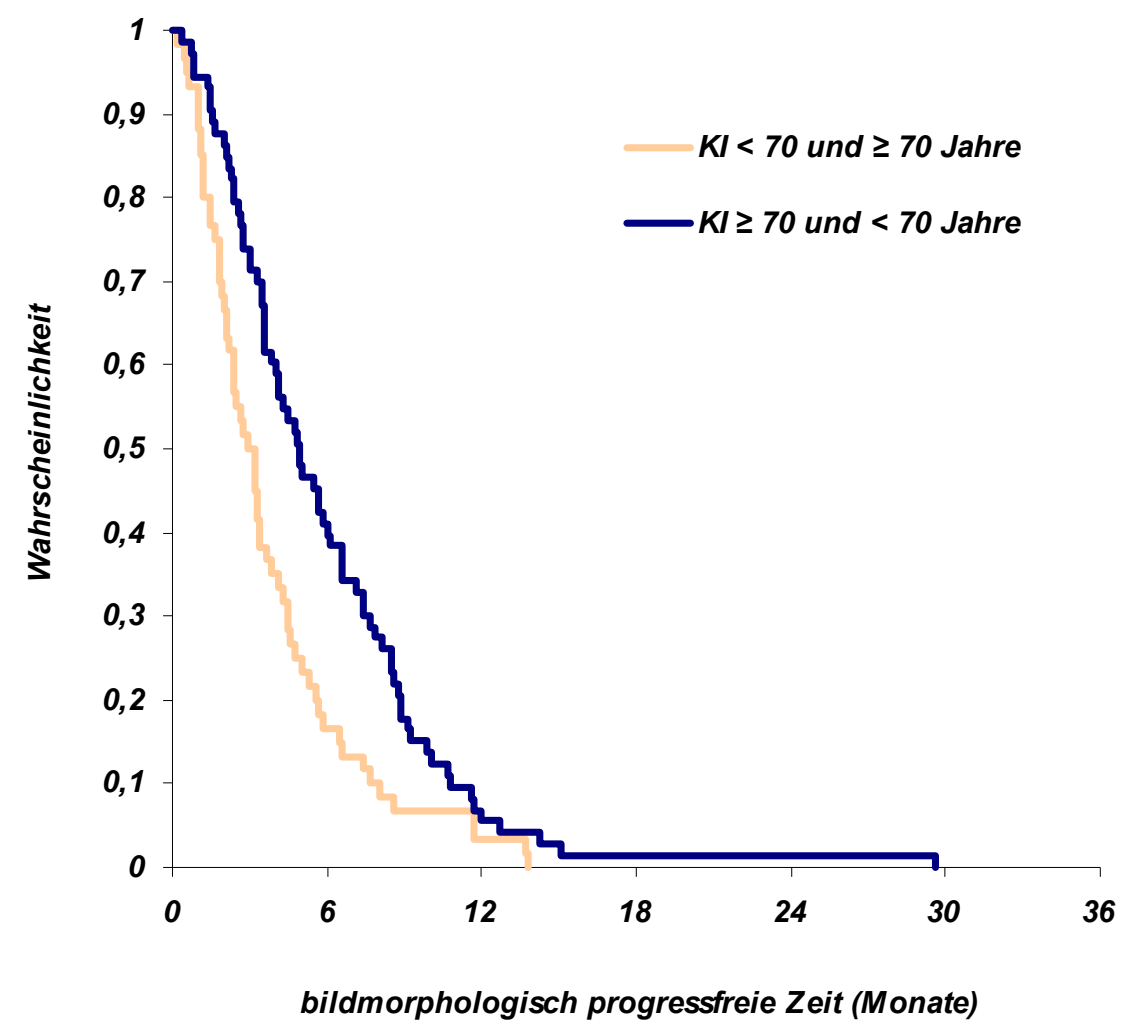

Abb.36: Progressionsanalyse für klinischen Progress korreliert mit Alter und präoperativem KI $(P=<0,01)$

\subsubsection{Progressionsanalyse in Abhängigkeit vom Resektionsausmaß}

Auch der Einflußfaktor Resektionsausmaß zeigte sich in unserem Patientenkollektiv von signifikanter Bedeutung, sowohl für klinische als auch für bildmorphologische Progression. So zeigte sich ein deutlicher Unterschied zwischen den makroskopisch vollständig resezierten Patienten, die ein medianes klinisch-progressfreies Intervall von 6,5 Monaten erreichten, und den lediglich biopsierten Patienten, bei denen das klinisch-progressfreie Intervall nur 2 Monate betrug. Bildmorphologisch konnte ein Tumorprogress für die makroskopisch vollständig resezierten Patienten, im Median nach 5 Monaten und für die Gruppe Biopsierter bereits nach 2,9 Monaten, ermittelt werden. 


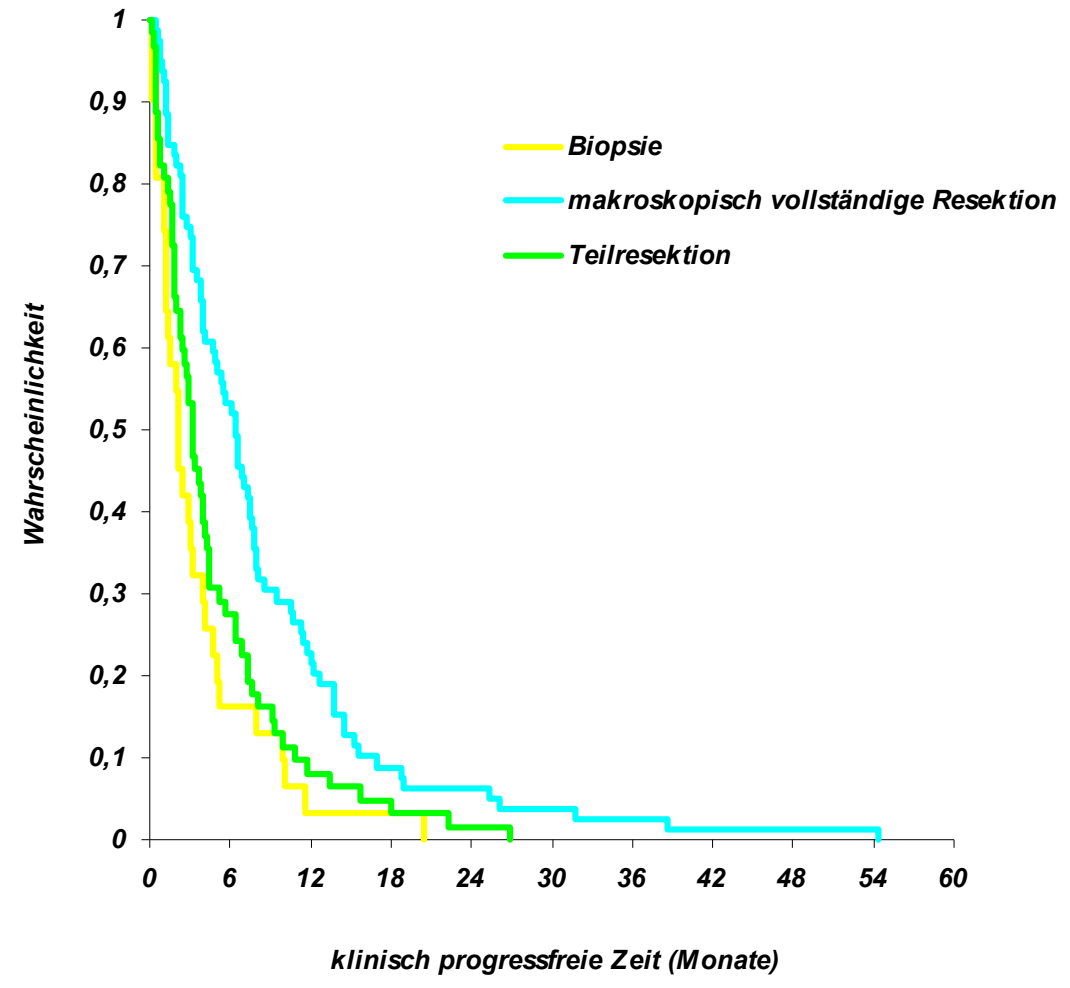

Abb.37: Progressionsanalyse für klinischen Progress in Abhängigkeit vom Resektionsausmaß $(P=<0,01)$

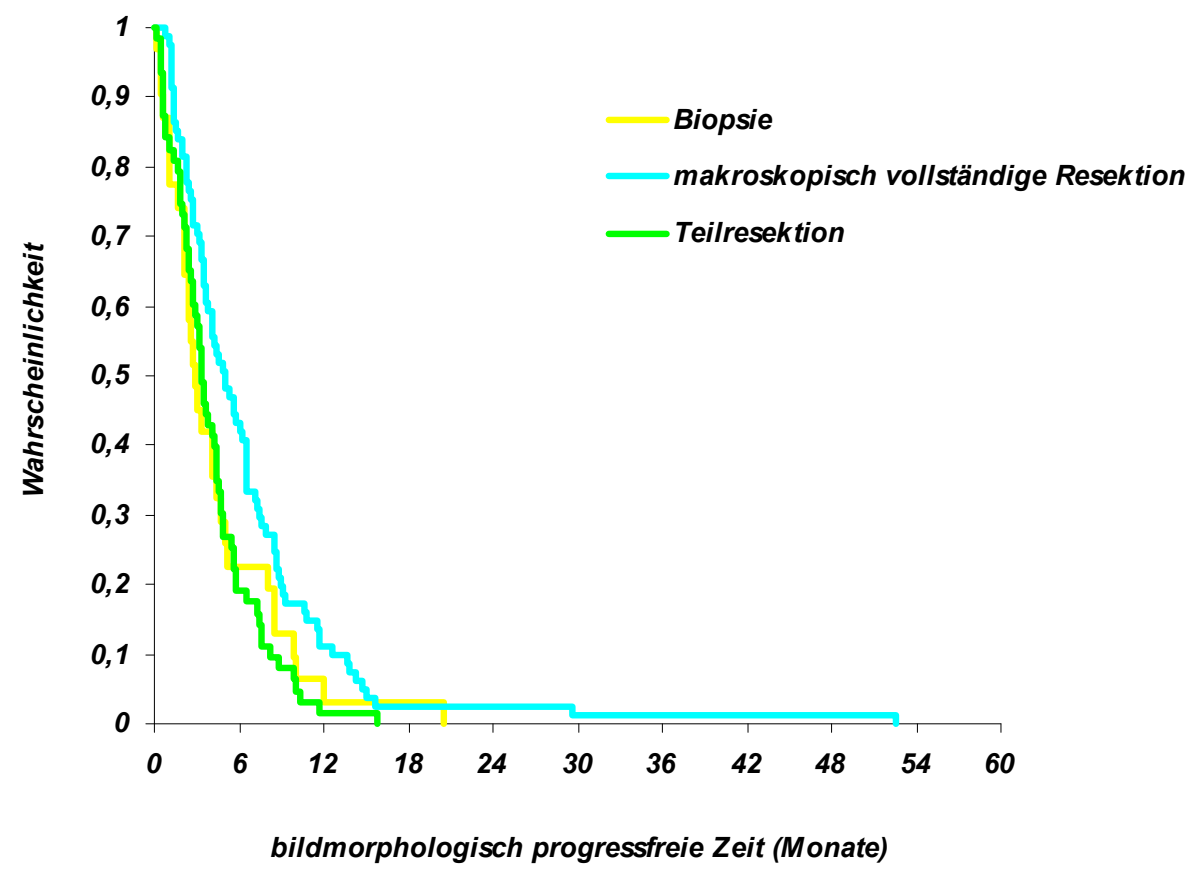

Abb.38: Progressionsanalyse für bildmorphologischen Progress in Abhängigkeit vom Resektionsaus$\operatorname{maß}(P=<0,01$ 
3.3.3 Progressionsanalyse in Abhängigkeit der postoperativen, adjuvanten Therapie Erfolgte eine Analyse nach unterschiedlichen postoperativen Therapien, zeigten sich signifikant unterschiedliche Ergebnisse, sowohl für den klinischen als auch für den bildmorphologischen Progress.

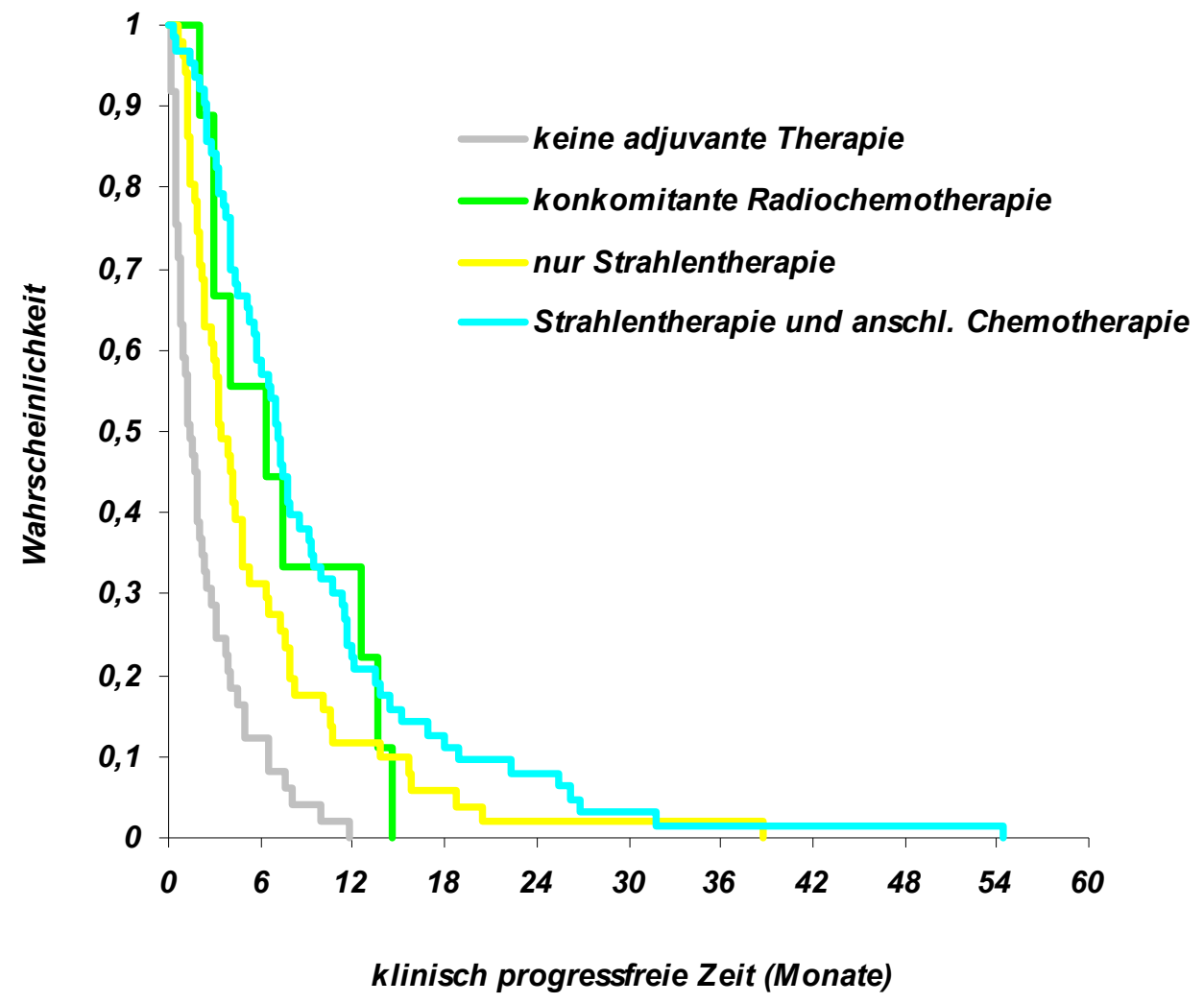

Abb.37: Progressionsanalyse klinisch in Abhängigkeit von der postoperativen Therapie $(P=<0,01)$ 


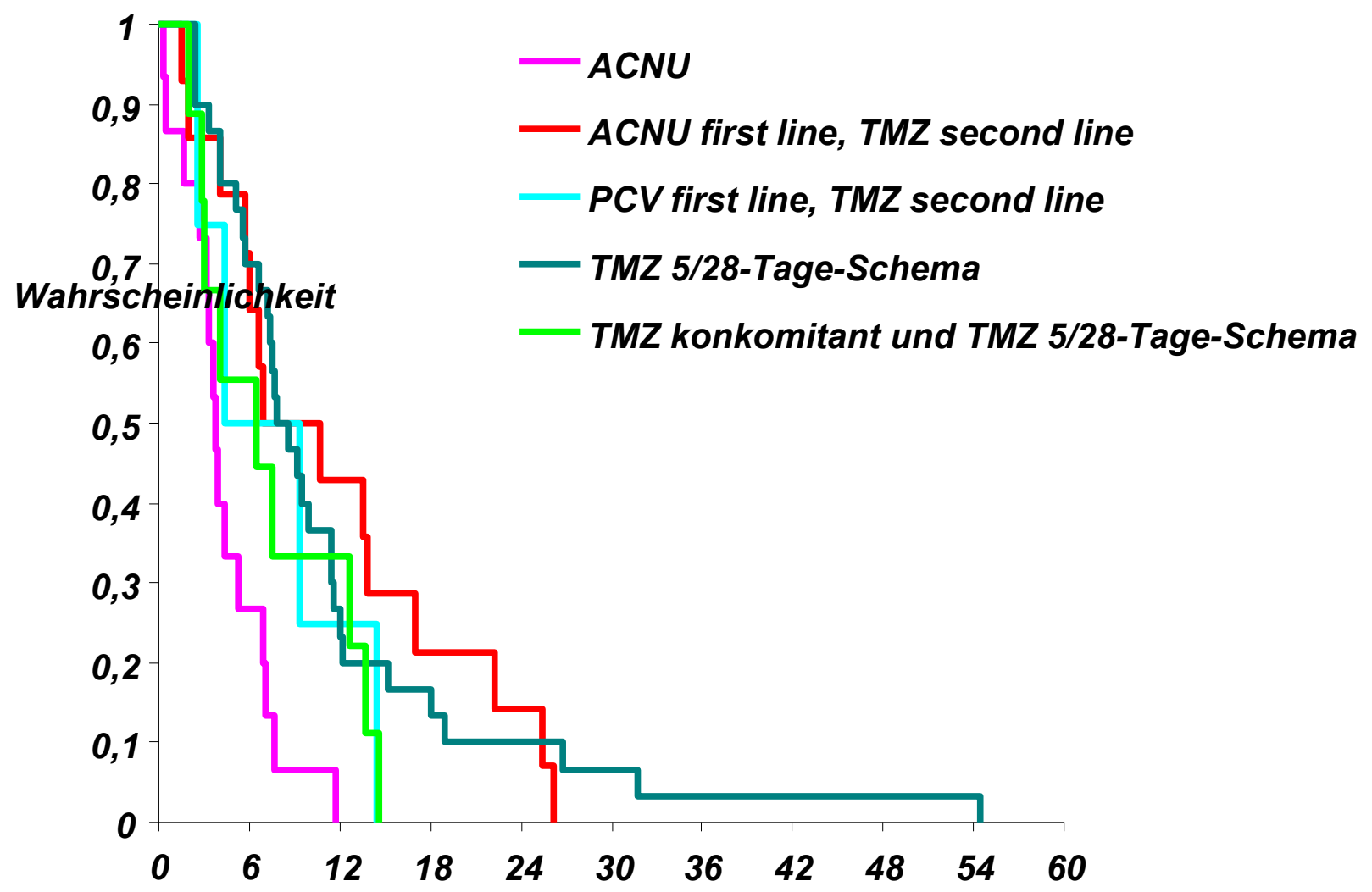

klinisch progressfreie Zeit (Monate)

Abb.36: Progressionsanalyse für klinischen Progress korreliert mit verschiedenen Chemotherapieregimen $(P=<0,01)$

\subsection{Langzeitüberlebende}

Insgesamt fanden sich 23 Patienten mit Gesamtüberlebenszeiten von 24 Monaten und länger. Das entspricht einem Anteil von 13\% der Gesamtpopulation. Von dieser Patientengruppe wurden $78 \%$ der Patienten makroskopisch vollreseziert und 22\% teilreseziert. Das mediane Alter lag bei 59 Jahren (Mittelwert 56 Jahre, Standardabweichung 10,94), der mediane Karnofsky-Index vor erster Operation bei 70 Punkten. 39\% dieser Patientengruppe unterzogen sich im Laufe der Erkrankung mindestens einer weiteren Operation nach Tumorprogress bzw. Tumorrezidiv, 17\% sogar einer dritten operativen Intervention. 96\% wurden postoperaiv bestrahlt, 22\% zeitgleich konkomitant mit Temozolomid chemotherapiert. 35\% erhielten als first-lineChemotherapie ACNU. 


\begin{tabular}{|lc|}
\hline Patienten mit Gesamtüberlebenszeit $\mathbf{Z}$ 2 Jahre & $\mathbf{N = 2 3}$ \\
\hline Medianes Alter & 59 \\
\hline Biopsie & $0(0 \%)$ \\
Subtotale Resektion & $5(22 \%)$ \\
Makroskopisch vollständige Resektion & $18(78 \%)$ \\
\hline 2. Operation & $9(39 \%)$ \\
3. Operation & $4(17 \%)$ \\
\hline Medianer Karnofsky-Index & 70 \\
Karnofsky-Index von-bis & $60-90$ \\
\hline Gesamtüberleben (Monate) & 43,5 \\
Mittelwert & 22,8 \\
Standardabweichung & $\mathbf{3 2}$ \\
Median & 19 \\
Klinisch-progressfreie Zeit (Monate) & 515 \\
Mittelwert & $\mathbf{1 4 , 6}$ \\
Standardabweichung & 10 \\
Median & 12,4 \\
Bildmorphologisch progressfreie Zeit (Monate) & $\mathbf{5}$ \\
\hline Mittelwert & $22(96) \%)$ \\
\hline Standardabweichung & $20(87 \%)$ \\
\hline Median & $3(13 \%)$ \\
\hline Strahlentherapie & $2(9 \%)$ \\
\hline First-line Chemotherapie & $6(26 \%)$ \\
\hline \hline Keine & $1(4 \%)$ \\
ACNU & $6(26 \%)$ \\
ACNU mit Wechsel auf TMZ & $5(22 \%)$ \\
\hline PCV mit Wechsel auf TMZ & \\
TMZ & \\
Konkomitant TMZ+TMZ & \\
\hline
\end{tabular}

Tab.7: Aufschlüsselung der Gruppe der Langzeitüberlebenden (Gesamtüberlebenszeit $\geq 24$ Monate)

\subsection{Signifikante Einflußgrößen}

In den folgenden Tabellen wurden die ermittelten signifikanten Ergebnisse der prognostisch- und therapeutisch günstigen Einflussfaktoren auf das Gesamtüberleben zusammengefasst. Es zeigte sich, dass Alter, initialer Karnofsky-Index und das Ausmaß der Tumorresektion in unserem Patientenkollektiv zu signifikant unterschiedlichen 1-Jahres-Überlebenswahrscheinlichkeiten führten. Dieses traf, insbesondere hinsichtlich der adjuvanten Therapieform, für die Gruppe der makroskopisch vollresezierten, aber auch für die Gruppe der nur teilresezierten Patienten zu. Insgesamt ergaben sich für Patienten unter 50 Jahren, mit einem Karnofsky-Status von über 70 Punkten und einer makroskopisch vollständigen Tumorresektion, anschließender konkomitanter Radiochemotherapie und angeschlossener Temozolomid Monotherapie, die höchsten 1-Jahres-Überlebenswahrscheinlichkeiten. 


\begin{tabular}{|c|c|c|c|c|}
\hline \multicolumn{5}{|c|}{$\begin{array}{l}\text { Gesamtüberleben } \\
\text { (signifikant günstige Einflussgrößen) }\end{array}$} \\
\hline Einflussgröße & Chi-Quadrat-Test & $P$ & Subgruppe & $\begin{array}{c}\text { 1-Jahres Überle- } \\
\text { benswahr- } \\
\text { scheinlichkeit (\%) }\end{array}$ \\
\hline Altersgruppen & 49,00 & $<0,0001$ & $<50$ Jahre & 62 \\
\hline Karnofsky-Index & 17,22 & 0,00018 & > 70 Punkte & 73 \\
\hline Resektionsausmaß & 30,61 & $<0,0001$ & $\begin{array}{l}\text { makroskopisch vollständige Re- } \\
\text { sektion }\end{array}$ & 53 \\
\hline $\begin{array}{l}\text { Kombination Alter und } \\
\text { Karnofsky-Index }\end{array}$ & 59,45 & $<0,0001$ & $K I \geq 70$ Punkte und Alter $<70$ Jahre & 57 \\
\hline $\begin{array}{c}\text { Adjuvante Therapie } \\
\text { insgesamt }\end{array}$ & 26,48 & $<0,0001$ & $\begin{array}{l}\text { TMZ konkomitant und anschl. } \\
\text { Monotherapie TMZ }\end{array}$ & 75 \\
\hline $\begin{array}{c}\text { Adjuvante Therapie bei } \\
\text { Teilresezierten }\end{array}$ & 14,147 & 0,00084 & $\begin{array}{l}\text { TMZ konkomitant und anschl. } \\
\text { Monotherapie TMZ }\end{array}$ & 60 \\
\hline $\begin{array}{l}\text { Adjuvante Therapie bei } \\
\text { makr. Vollresezierten }\end{array}$ & 31,74 & $<0,0001$ & $\begin{array}{l}\text { TMZ konkomitant und anschl. } \\
\text { Monotherapie TMZ }\end{array}$ & 86 \\
\hline $\begin{array}{l}\text { Jahrgänge vor und nach } \\
\text { Einführung der konko- } \\
\text { mitanten Radiochemo- } \\
\text { therapie }\end{array}$ & 4,55 & 0,0328 & $\begin{array}{l}\text { Nach Einführung der konkomitan- } \\
\text { ten Radiochemotherapie }\end{array}$ & 43 \\
\hline
\end{tabular}

Tab.10: Signifikante Ergebnisse für die einzelnen Subgruppenanalysen zusammengefasst. 


\section{Diskussion}

Das Glioblastom ist der häufigste hirneigene Tumor im Erwachsenenalter. Bis heute erfordert der schnelle und fatale Verlauf dieser Erkrankung ständig neue randomisierte prospektive Studien zur Verbesserung der Überlebenszeit der Patienten. Die Studienergebnisse führten immer wieder zu Neuerungen in den aktuellen Therapieempfehlungen. Die Umsetzung dieser Erkenntnisse in der Uniklinik Göttingen zog in den Jahren 1998 bis 2004 verschiedene Therapiemodalitäten nach sich, welche sich grundsätzlich an großangelegten prospektiven, randomisierten Studien orientierten. In der vorliegenden retrospektiven Untersuchung wurden die Ergebnisse verschiedener Behandlungsstrategien aufgearbeitet, miteinander verglichen und mit der Datenlage der internationalen Literatur in Beziehung gesetzt.

\subsection{Das Patientenkolllektiv}

Das untersuchte Patientenkollektiv setzte sich aus den in den Jahren 1998 bis 2004 in Göttingen histologisch neudiagnostizierten Patienten mit Glioblastomen zusammen. Da die histologische Diagnosesicherung das entscheidende Einschlusskriterium war, wurden die Patienten, bei denen sich bildmorphologisch der dringende Verdacht auf ein Glioblastom ergab, jedoch der Allgemeinzustand oder die Patientenentscheidung keinen operativen Eingriff zuließ, nicht erfasst. Außerdem wurden die Patienten, deren Krankenblatt nicht bezogen werden konnte, oder in deren Krankenblatt die Dokumentation so lückenhaft war, dass der Einschluss in die Datenanalyse nicht sinnfoll erschien, nicht mit eingeschlossen.

In der Beschränkung des Patientenkollektivs auf neudiagnostizierte Glioblastome unterschied sich diese Arbeit von einer Reihe anderer Untersuchungen, bei denen Glioblastome gemeinsam mit anaplastischen Astrozytomen und den chemosensitiveren Oligigodendrogliomen untersucht wurden (Shapiro1992, Medical Research Council Brain Tumor Working Party 2001, GMT Group 2002).

\subsection{Alter und Karnofsky-Index zum Zeitpunkt der Erstdiagnose}

Die 2005 von Stupp et al. veröffentlichte prospektive Studie zur Wirksamkeit der konkomitanten Temozolomidtherapie hingegen fokussierte sich ebenfalls ausschließlich auf ein Patientenkollektiv erstdiagnostizierter Glioblastome. Da Patienten über eine Altersgrenze von 70 Jahren hinaus nicht in letztere Studie eingeschlossen wurden, war das mediane Lebensalter von 56 Jahren deutlich niedriger als das mediane Alter in unserer Therapiebeobachtung mit 63 Jahren. Auch im Vergleich zu dem in der Li- 
teratur beschriebenen Altersgipfel zwischen 50 und 60 Jahren lag der Altersgipfel in unserem nicht randomisierten Patientenkollektiv höher. Der jüngste Patient in unserer Untersuchung war 30 Jahre alt, der älteste Patient 85 Jahre. Das Alter eines Patienten war neben dem Karnofsky-Index zum Zeitpunkt der Erstdiagnose und dem Ausmaß der Tumorresektion ein wichtiger Einflussfaktor hinsichtlich der Überlebenszeit. Der initiale mediane Karnofsky-Index des Gesamtkollektivs vor der ersten Operation betrug $70 \%$, der schlechteste $20 \%$. Der Großteil der von uns retrospektiv untersuchten Patienten befand sich also zum Zeitpunkt der Diagnosestellung in einem klinischen Zustand der Selbstversorgung, war aber nicht in der Lage, Arbeit oder andere Aktivitäten normal zu verrichten. Oft waren ein fortgeschrittenes Alter (über 70 Jahre) und ein vergleichsweise schlechter Karnofsky-Index (kleiner als 70 \%) Ausschlusskriterien für prospektive, randomisierte Studien, so dass diese Patientengruppen in vielen Analysen keine Repräsentation fand. In unserer Untersuchung lag der Anteil der über $70-J a ̈ h r i g e n ~ b e i ~ 34 \%$. Auch der Anteil der Patienten, die einen geringeren Karnofsky-Index als $70 \%$ hatten, war bei unserem Gesamtkollektiv mit $45 \%$ ebenfalls stark vertreten. In unserem nicht randomisierten Patientenkollektiv zeigte sich, wie in der Literatur beschrieben, dass insbesondere junge Patienten und auch Patienten mit einem hohen Karnofsky-Index zum Zeitpunkt der Diagnosestellung insgesamt die besten Überlebenswahrscheinlichkeiten aufwiesen. Im Rahmen der Survivalanalysen konnten für den Faktor Alter hochsignifikante Unterschiede, sowohl für die Gesamtüberlebenszeit als auch für die klinisch-progressfreie Überlebenszeit und die bildmorphologisch-progressfreie Zeit dargestellt werden. Für den Faktor Karnofsky-Index fanden sich signifikante Unterschiede für das Gesamtüberleben, jedoch nicht für die progressfreie Zeit.

\subsection{Die Operation}

Der erste Schritt bei der Behandlung hochgradiger Gliome, nach neuroradiologischer Verdachtsdiagnose, stellte die mikrochirurgische, möglichst vollständige Resektion dar. Das Ziel der Operation war zum einen die Diagnosesicherung durch Gewinnung einer Gewebeprobe und die Reduktion der raumfordernden Tumormasse, ohne Beeinträchtigung der klinisch-neurologischen Funktion. Aufgrund der mangelnden prospektiven Studienlage galt es teilweise als umstritten, ob die weitestgehende Resektion einen signifikanten Überlebensvorteil erbringt. Hart et al. verglichen retrospektiv im Rahmen einer Metaanalyse biopsierte und operierte Patienten mit malignem Gliom; Sie behaupteten, dass die mittlere Überlebenszeit sich durch die 
radikale Operation nicht wesentlich verlängert (Hart et al. 2000). Aktuellere Untersuchungen, insbesondere bezogen auf fluoreszenzgestützte Tumorresektion, zeigten hingegen, dass das rezidivfreie Intervall wesentlich von einer bildmorphologisch vollständigen Tumorresektion abhängig ist (Stummer et al. 2003). Zudem haben postoperative funktionelle Untersuchungsmethoden wie PET und SPECT gezeigt, dass selbst bei radikaler Operation, aufgrund des infiltrativen Tumorwachstums, Tumorreste verbleiben und zur unvermeidbaren Rezidivierung der malignen Gliome führen (Weber et al. 1997, Gross et al. 1998). Zudem ist es weiterhin auch aktuell umstritten, ob der fehlende Nachweis eies Kontrastmittel aufnehmenden Resttumors definitiv postoperativ verbliebenes aktives Tumorgewebe ausschließt. So haben Untersuchungen mit Aminosäure-PET gezeigt, dass trotz fehlendem Resttumornachweis im MRT aktives Tumorgewebe mittels PET nachweisbar sein kann. Die hier dokumentierten Resektionsausmaße bezogen sich primär auf den schriftlichen Operationsbericht. Diese Angaben waren ohne direkte postoperative MRT-Kontrolle schwer objektivierbar, fanden jedoch in den Klassifizierungen ,makroskopisch weitestgehende Resektion' und ,subtotale Resektion' eine adäquate Einteilung.

In unseren Analysen zeigte sich ein deutlicher Überlebensvorteil der Patienten, die eine weitestgehende Operation erhielten, gegenüber Patienten, bei denen nur eine Teilresektion angestrebt werden konnte, und diese wiederum zeigten längere Überlebenszeiten als die biopsierten Patienten. Diese Ergebnisse waren statistisch hochsignifikant ( $p=6,27 \mathrm{E}-7)$, wobei man beachten muss, dass die Entscheidung über die operative Maßnahme nicht prospektiv randomisiert erfolgte, sondern die behandelnden Ärzte zusammen mit dem Patienten die Nutzen und Risiken des Ausmaßes des Eingriffes berieten. Dabei wurden Patienten mit schlechtem Allgemeinzustand und fortgeschrittenem Alter eher biopsiert, insbesondere wenn der Allgemeinzustand oder die Lokalisation des Tumors limitierend war. Bei eloquenter Tumorlage konnte sich das Ausmaß der Operation nur auf eine Teilresektion beschränken. Betrachten wir die Altersverteilung, so lag in den Gruppen der vollresezierten und teilresezierten Patienten das mediane Alter bei 63 Jahren, in der Gruppe der biopsierten Patienten bei 67 Jahren. Bei der Verteilung der Karnofsky-Indizes war ebenfalls in der Gruppe der vollresezierten Patienten der beste mediane Karnofsky-Index mit 70 Punkten zu verzeichnen. Die Gruppen der teilresezierten und biopsierten Patienten hatten einen medianen präoperativen Karnofsky-Status von 60 Punkten. Dieses reflektierte die Gründe für das operative Vorgehen. 
Bei den Patienten, die nicht vollreseziert wurden, zeigte sich zudem eine große Zurückhaltung in der Anwendung von adjuvanten Therapien. In der Gruppe der teilresezierten lag der Anteil der Patienten, die nicht adjuvant therapiert wurden bei 42 Prozent und bei 33 Prozent in der Gruppe, der biopsierten Patienten. In der Gruppe der vollresezierten Patienten lag der Anteil der Patienten, die keine adjuvante Therapie erhielten, bei lediglich 14\%. Dieses zeigt, dass in der Gruppe der makroskopisch vollständig resezierten Patienten das Kollektiv zu finden war, das für weiterführende Therapien aufgrund von Tumorlokalisation, neurologischem Zustand und Alter am besten geeignet schien und damit vermutlich weitere positive Prognostikfaktoren auf sich vereinigte. In einer weiteren Analyse bildeten wir 8 Vergleichsgruppen, indem wir zwischen Patienten mit makroskopischer Vollresektion und verbliebenem Resttumor unterschieden und diese beiden Gruppen nochmals nach der angewandten adjuvanten Therapie unterteilten (keine adjuvante Therapie, nur Radiatio, Radiochemotherapie und Radiatio mit anschließender Chemotherapie). Betrachteten wir nun den Einfluß vom Resektionsausmaß auf das Gesamtüberleben stellten wir fest, dass bei den Patienten, die dieselbe adjuvante Therapie erhielten, die Gruppe der vollresezierten Patienten immer einen Vorteil im Gesamtüberleben gegenüber der Patienten mit verbliebenem Resttumor hatten. In unserem Patientenkollektiv beobachteten wir außerdem, dass die Art der adjuvanten Therapie, bezogen auf das Gesamtüberleben, scheinbar von größerer Bedeutung war, als das Resektionsausmaß. So schnitten z.B. die Patienten, die makroskopisch vollreseziert waren und keine adjuvante Therapien erhielten, beinahe ebenso schlecht ab wie die Patienten, die einen Resttumor hatten und ebenfalls keine adjuvante Therapie erhielten. Beide Gruppen hatten eine 1-Jahres-Überlebenswahrscheinlichkeit von 0 Prozent. Bei den Patienten, die eine alleinige Strahlentherapie erhielten, hatten die Patienten mit verbliebenem Resttumor eine 1-Jahres-Überlebenswahrscheinlichkeit von 12\% und die Patienten, die makroskopisch vollreseziert wurden, eine 1-JahresÜberlebenswahrscheinlichkeit von 31\%. Deutlich bessere Ergebnisse zeigten die Patienten, die eine adjuvante Chemotherapie erhielten. Hier lagen die 1-Jahres Überlebenswahrscheinlichkeiten zwischen $45 \%$ bei den Patienten, die einen Resttumor hatten und konkomitant behandelt wurden, und 86\% in der Gruppe, die makroskopisch vollreseziert wurde und eine konkomitante Chemotherapie erhielten. $\mathrm{Pa}$ tienten mit Resttumor, die eine Radiatio mit anschließender Chemotherapie erhielten, zeigten eine 1-Jahres-Überlebenswahrscheinlichkeit von $50 \%$ und gleichbehandelte Patienten mit vorangegangener Vollresektion sogar $67 \%$. 


\subsection{Postoperative Therapie}

Aufgrund der unterschiedlichen Therapiemodalitäten, die in den Jahren 1998 bis 2004 gemäß aktueller Therapieempfehlung angewandt wurden, ergaben sich die unterschiedlichen Vergleichsgruppen für unsere Untersuchungen.

53 Patienten haben eine alleinige Strahlentherapie erhalten, mit Strahlentherapie und adjuvanter Chemotherapie wurden 64 Patienten behandelt. Die Gruppe, der mit konkomitanter Temozolomidtherapie behandelten Patienten, war mit 12 Patienten relativ klein, da diese Therapie erst seit dem Jahr 2004 angewandt wurde. Einen großen Anteil am Gesamtkollektiv machten die Patienten aus, die keinerlei adjuvante Therapie erhielten. Dies betraf 49 Patienten.

\subsubsection{Strahlentherapie}

Die Standardtherapie für maligne Gliome beinhaltet neben der Resektion bisher die Strahlentherapie als generelle Behandlungsstrategie. Sie gilt als die effektivste, nicht chirurgische, palliative Maßnahme. 1978 wurde eine signifikante Verlängerung der Lebenserwartung durch Walker et al. beschrieben. Das mediane Überleben konnte durch postoperative Radiatio von 14 auf 36 Wochen verlängert werden. In unseren Untersuchungen haben 49 Patienten des Gesamtkollektivs keine Strahlentherapie erhalten und 53 Patienten wurden einer Radiatio unterzogen. Sowohl die Prognosefaktoren, Alter und präoperativer Karnofsky-Status als auch das Resektionsausmaß waren homogen verteilt. Es zeigte sich, dass die Patienten, die keine postoperative Strahlentherapie erhielten, ein medianes Überleben von 2 Monaten und eine 1Jahres-Überlebenswahrscheinlichkeit von $1 \%$ hatten. Hingegen kamen die Patienten, die eine postoperative Radiatio erhielten, auf ein medianes Überleben von 5 Monaten und einer 1-Jahres-Überlebenswahrscheinlichkeit von 20\%. Der Grund für das schlechtere Ergebnis, hinsichtlich der medianen Überlebenszeit nach Bestrahlung in unseren Ergebnissen, bezogen auf die Ergebnisse von Walker et al.1978 war sicherlich auch in dem relativ hohen medianen Alter unserer Vergleichsgruppe zu finden. So betrug das mediane Alter unserer bestrahlten Patienten 67 Jahre, hingegen betrug das mediane Alter in der entsprechenden Vergleichsgruppe der herangezogenen Studie von Walker et al., 56 Jahre. Untersuchungen ergaben, dass vor allem jüngere Patienten von der Strahlentherapie profitierten (Walker et al. 1979). Ein wichtiger Fortschritt für die Verträglichkeit der Strahlentherapie war die Erkenntnis, dass, aufgrund der hohen lokalen Rezidivwahrscheinlichkeit, die Ganzhirnbestrahlung kei- 
nen Vorteil gegenüber der Bestrahlung der erweiterten Tumorregion brachte (Hess 1994). Verschiedenste Fraktionierungsschemata haben bisher keine Überlegenheit zur herkömmlichen Applikation von einer Gesamtdosis von 54-60 Gy in 1,8-2 Gy Fraktionen gezeigt (Werner-Wasik et al. 1996). Von unserem Patientenkollektiv erhielten 54\% eine adjuvante Strahlentherapie, lediglich $11 \%$ des Gesamtkollektivs mussten die begonnene Therapie aufgrund gravierender Verschlechterung des Allgemeinzustandes abbrechen. 7\% der Patienten erhielten komkomitant Temozolomid während der Radiatio. 28\% des Gesamtkollektivs wurden nicht bestrahlt.

\subsubsection{Chemotherapie}

Im ersten Schritt untersuchten wir die unterschiedlichen adjuvanten Therapieformen, ohne die verschiedenen Chemotherapeutika zu differenzieren. Es wurden vier Gruppen definiert (keine adjuvante Therapie, ausschließlich Strahlentherapie, Strahlentherapie mit anschließender Chemotherapie und konkomitante Therapie). Betrachteten wir die Überlebenswahrscheinlichkeiten unserer Daten, stellten wir fest, dass sie sehr unterschiedlich sind ( $\mathrm{p}=2,41 \mathrm{E}-13)$. In der Gruppe der nicht adjuvant therapierten Patienten betrug die 1-Jahres-Überlebenswahrscheinlichkeit gerade 1\%, in der Gruppe der ausschließlich radiotherapierten Patienten 20\%. Die Patienten, die eine adjuvante Chemotherapie erhielten, erreichten eine 1-Jahres-Überlebenwahrscheinlichkeit von $61 \%$ und die konkomitant behandelten Patienten von $75 \%$. Bei diesen eindrucksvollen Daten galt zu berücksichtigen, dass die Prognosefaktoren nicht homogen verteilt waren. Die ältesten Patienten befanden sich in den Gruppen, die entweder keine Therapie erhielten oder nur strahlentherapeutisch behandelt wurden. In diesen beiden Gruppen lag das mediane Alter zwischen 71 Jahren für die Patienten, die keine Therapie erhielten und 67 Jahren bei den Patienten, die ausschließlich eine Strahlentherapie erhielten. Bei den Gruppen, bei denen zusätzlich eine Chemotherapie durchgeführt wurde, lag das mediane Alter bei 59 Jahren. Auch bei der Betrachtung der Karnofsky-Indizes fällt auf, dass die schlechtesten Punktwerte, nämlich im Mittel 60 Punkte in den Gruppen der nicht behandelten Patienten und in der Gruppe der ausschließlich bestrahlten Patienten vorzufinden waren. Die Patienten, die eine adjuvante Chemotherapie erhielten, hatten jeweils einen Karnofsky-Index von 70 im Mittel. Es zeigten sich also deutlich schlechtere Prognosefaktoren für die Patienten, die keine Therapie oder ausschließlich Radiatio erhielten, so dass bei dem Vergleich aller Therapieoptionen, die unterschiedlichen Ausgangssituationen berücksichtigt werden mussten. 
Vergleicht man im Weiteren dann die Prognosefaktoren nur innerhalb der verschiedenen Chemotherapie Gruppen, ließ sich eine sehr homogene Verteilung der Prognosefaktoren Alter und Karnofsky-Index feststellen. Einzig in der Gruppe der ACNU therapierten, die im Folgenden noch Temozolomid erhielten, fiel ein jüngeres medianes Alter auf. Gerade bei jungen Patienten war der maximale Therapiewunsch, bei schlechtem Ansprechen der primären Chemotherapie, besonders stark ausgeprägt. Die ansonsten homogene Verteilung von Karnofsky-Index und medianem Alter innerhalb der verschiedenen Gruppen der adjuvanten und konkomitanten Chemotherapie ließ sich dadurch erklären, dass bei einer chemotherapeutischen Behandlung, die oftmals eine zusätzliche Belastung für den Patienten darstellt, das behandelnde Ärzteteam die Indikation zur Behandlung nur bei stabilem Allgemeinzustand stellte. Für unsere folgenden Untersuchungen ist es wichtig festzuhalten, dass sich die Gruppen der Patienten, die eine adjuvante Chemotherapie erhielten in der Verteilung der wichtigsten Prognosefaktoren nicht wesentlich voneinander unterschieden, so dass durch den Vergleich der verschiedenen eingesetzten Chemotherapieprotokolle ein Rückschluss auf den Effekt des einzelnen Medikaments möglich war.

Stewart untersuchte 2002 in einer Meta-Analyse von 12 randomisierten Studien die Effizienz der adjuvanten Chemotherapie. In dieser Studie wurde die Verbesserung der 1-Jahres Überlebenswahrscheinllichkeit durch Nitroseharnstoffe zusätzlich zur Bestrahlung von $40 \%$ auf $46 \%$ bei einem Patientenkollektiv höher maligner Gliome beschrieben. Es gebe jdoch keinen Anlass diese Daten nicht auf die Gruppe der Glioblastome zu übertragen (Stewart 2002). Bei unserem Patientenkollektiv, welches sich ausschließlich auf Patienten mit neu diagnostiziertem Glioblastom bezog, hatten die Patienten, die mit ACNU nach Strahlentherapie behandelt wurden, eine 1-Jahres Überlebenswahrscheinlichkeit von 27\%. Die ausschließlich bestrahlten Patienten hatten hingegen nur eine 1-Jahres-Überlebenswahrscheinlichkeit von $20 \%$. Auch die mediane Überlebenszeit war in der Gruppe, der mit ACNU behandelten Patienten, mit 9 Monaten, der medianen Überlebenszeit der ausschließlich bestrahlten Patienten, die 5 Monate beträgt, deutlich überlegen. Somit stützten wir die Behauptung von Stewart et al., dass die zusätzliche Anwendung von Chemotherapie, auch die Anwendung der Nitroseharnstoffe, der alleinigen strahlentherapeutischen Behandlung maligner Glioblastome überlegen ist. Allerdings muß erwähnt werden, dass im Gegensatz zu unseren Untersuchungen die Verbesserung der Überlebenszeit durch eine zusätzliche Therapie mit Nitroseharnstoffe sich in zahlreichen randomisierten 
Kooperations-Studien als nicht signifikant erwies (Walker et al. 1978, Green et al. 1983, Chang et al. 1983, Shapiro 1989, Medical Research Council trial 2001).

Seit 2004 wird in Göttingen Temozlomid konkomitant angewandt. Bei dem Vergleich der Temozolomid- Monotherapie nach Radiatio und der konkomitante Therapie zeigte sich nach unserer Datenlage kein signifikanter Unterschied bezüglich der Überlebenswahrscheinlichkeit $(p=0,39)$. Die mediane Überlebenszeit bei den konkomitant behandelten Patienten betrug 17 Monate. Dieser Gruppe vergleichbar, bezüglich der prognostischen Faktoren Alter und Karnofsky-Index, war in unserem Patientenkollektiv die Gruppe der bestrahlten und anschließend chemotherapierten Patienten. In dieser Gruppe betrug das mediane Überleben 15 Monate. Obwohl die Gruppe der konkomitant behandelten Patienten schlechter gestellt war in Hinblick auf die Verteilung von Vollresektion, Teilresektion und Biopsie, zeigten die konkomitant behandelten Patienen ein um 2 Monate verlängertes medianes Überleben im Vergleich zu den nach Radiotherapie chemotherapierten Patienten. Die konkomitante Therapie mit Temozolomid zeigte in einer EORTC- Studie von 2005 eine signifikante Steigerung der Lebenserwartung gegenüber der allein strahlentherapierten Patienten. In dieser Studie wurde das mediane Überleben der konkomitanten Temozolomid auf 14.6 Monate angegeben und das in der Gruppe der alleinig Strahlentherapierten auf 12,1 Monate. Stupp et al postulieren, dass die konkomitanten Chemotherapie eine bedeutende, statistisch signifikante Neuerung in der Behandlung von Glioblastomen darstellt. Vergleichen wir, in Anlehnung an die Studie von Stupp et al., die Gruppen der konkomitant behandelten Patienten mit den ausschließlich bestrahlten Patienten, differierten die medianen Überlebenszeiten stark zugunsten der konkomitant behandelten Patienten. So betrug die mediane Überlebenszeit der ausschließlich bestrahlten Patienten 5 Monate, die der konkomitant behandelten Patienten 17 Monate. Auch die mediane radiographisch-progressionsfreie Überlebenszeit war in der Gruppe der konkomitant behandelten Patienten mit 6 Monaten der progressionsfreien Überlebenszeit, der ausschließlich bestrahlten Patienten, mit 4 Monaten deutlich überlegen. Ergänzend muß man festhalten, dass diese beiden Gruppen aufgrund des retrospektiven Charakters unserer Analysen, hinsichtlich der Prognosefaktoren und des Resektionsausmaßes, sehr unterschiedlich und zu gunsten der konkomitant behandelten Patienten zusammengesetzt waren und somit ein Vergleich nur eine eingeschränkte Beurteilung zuließ. Bemerkenswert ist jedoch, dass das mediane Überleben der konkomitant behandelten Patienten an der Universitätsklinik Göttingen die mediane Überlebenszeit in der EORTC Studie um 2,4 Monate überstieg. Obwohl das 
mediane Alter in der herangezogenen Vergleichsstudie mit 56 Jahren 3 Jahre jünger ausfiel als bei unseren konkomitant behandelten Patienten, verglichen wir unsere Ergebnisse der verschiedenen adjuvanten Chemotherapien (ACNU, ACNU/TMZ, Temozolomid Monotherapie) mit der konkomitanten Behandlung und stellten fest, dass die Patienten, die zuerst mit ACNU behandelt wurden und anschließend Temozolomid erhielten, das beste mediane Überleben mit 19 Monaten erreichten. Diese Gruppe wies allerdings auch das jüngste mediane Alter auf. Hinsichtlich initialem Karnofsky-Index unterschieden sich die Gruppen nicht, das mediane Alter lag in allen Gruppen zwischen 59 und 63 Jahren. Eine Ausnahme bildete die Gruppe mit den primär mit ACNU und sekundär mit Temozolomid behandelten Patienten, mit einem medianen Alter von 49 Jahren.In der Gruppe der konkomitant mit TMZ behandelten Patienten betrug das mediane Überleben 17 Monaten. Die Monotherapie mit TMZ erbrachte eine mediane Überlebenszeit von 16 Monaten, bei ACNU Monotherapie wurde eine mediane Überlebenszeit von 9 Monaten erreicht. Verglich man die konkomitante Temozolomidtherapie mit der Temozolomidmonotherapie, bezüglich der Überlebenswahrscheinlichkeiten im Kaplan-Meyer-Diagramm, zeigten die beiden Kurven keine statistisch signifikante Abweichung voneinander $(p=0,39)$. Auch die Gegenüberstellung von den ACNU therapierten Patienten und den mit Temozolomid nach Strahlentherapie behandelten Patienten ergab keinen statistisch signifikanten Unterschied in der Überlebenswahrscheinlichkeit $(p=0,88)$. Auch die radiographisch gesicherte progressionsfreie Überlebenszeit betrug sowohl bei den konkomitant behandelten Patienten als auch bei den Patienten die Temozolomid als Monotherapie erhielten und bei denen, die nach ACNU auf Temozolomid geschwenkt waren, jeweils 6 Monate. Einzig die Patienten, die ACNU als Monotherapie erhielten, schnitten mit 4 Monaten schlechter ab. Die Patienten, die keine adjuvante Chemotherapie erhielten hatten ein medianes radiographisch-progressfreies Überleben von 2 Monaten. Obwohl die Daten aus dem Vergleich der verschiedenen Chemotherapien keine statistisch signifikanten Unterschiede bezüglich der 1-Jahres-Überlebenswahrscheinlichkeit und der progressionsfreien Überlebenszeit ergaben, können wir festhalten, dass mit der konkomitanten Temozolomidtherapie die bisher besten Ergebnisse bezüglich der medianen Überlebenszeit und der 1-und 2-Jahres Überlebenswahrscheinlichkeit erzielt wurden. Seit 2004 wurde in der Göttinger neurochirurgischen onkologischen Ambulanz die konkomitante Temozolomidtherapie als Therapie der ersten Wahl eingesetzt. Auch diese Patienten sind und werden weiter in dieser Datenbank erfasst. Es wird interessant sein zu untersuchen, inwieweit unsere Hoff- 
nungen auf die Überlegenheit der konkomitanten Temozolomidtherapie in einem größeren Patientenkollektiv doch noch statistische Signifikanz erreicht.

\section{Zusammenfassung}

Trotz umfangreicher klinischer Forschung auf der Ebene der operativen, strahlentherapeutischen und chemotherapeutischen Möglichkeiten, hat sich die Prognose für Patienten mit einem Glioblastoma multiforme in den letzten Jahren nur geringfügig verbessert. In der Universitätsklinik Göttingen, sowie auch in vielen anderen Neurochirurgischen Zentren basieren die angewendeten chirurgischen und adjuvanttherapeutischen Maßnahmen auf internationalen Studien homogener Patientengruppen, welche durch umfangreiche und langjährige Erprobung von Therapiemodalitäten allgemeine Empfehlungen zur Behandlung dieses hochmalignen Hirntumors darstellen. Einerseits sind diese Therapiestrategien einem ständigen Wandel durch neue wissenschaftliche Erkenntnisse unterzogen, und andererseits werden die Entscheidungen, bezüglich der individuellen Ausweitung der adjuvanten Therapien, in Abhängigkeit einer Vielzahl von patientenbezogenen Einflussfaktoren getroffen. Somit ist ein strukturierter Vergleich der verschiedenen Therapiemodalitäten im klinischen Alltag retrospektiv nur schwer realisierbar und bedarf als Mindestvoraussetzung einer suffizienten Nachbeobachtung der behandelten Patienten. Um eine Reevaluation und Qualitätskontrolle der Therapiestrategien eines großen Patientenkollektivs in der Universitätsklinik Göttingen zu realisieren, wurde eine TumorpatientenDatenbank entwickelt, die neben der Möglichkeit der retrospektiven Datenanalyse auch als hilfreiches Mittel zur ambulanten Patientenbetreuung genutzt wird. Hier wurden alle relevanten Daten zum gesamten Krankheitsverlauf der einzelnen Patienten dokumentiert und schließlich für einen Vergleich mit den jeweils zeitgemäßen Therapieregimen aus internationalen Studien herangezogen. Ziel dieser Untersuchung war es also, die bereits durchgeführten Therapiemodalitäten in den Jahren von 1998 bis 2004, hinsichtlich der Gesamtüberlebenszeit und progressfreien Überlebenszeit, auszuwerten und prognostische Einflussfaktoren, wie Alter, klinischer präoperativer Status sowie das Ausmaß der Tumorresektion mit einzubeziehen In unserer retrospktiven Analyse wurden 180 Patienten untersucht, deren einziges Einschlusskriterium das histologisch gesicherte Glioblastoma multiforme darstellte. Die zu untersuchenden Daten wurden mit Hilfe der MS Access Datenbank gesam- 
melt und mit Winstat und Excel bearbeitet. Bei unserem Gesamtkollektiv betrug das mediane Alter 63 Jahre, der mediane Karnofsky-Index hatte einen Punktwert von 70. Bei 47\% aller Patienten konnte initial eine makroskopisch vollständige Tumorresektion durchgeführt werden. In 53\% der Fälle erfolgte aufgrund der Lokalisation in eloquenten Arealen oder aufgrund der Ausdehung des Tumors nur eine subtotale Resektion oder Biopsie zur Diagnosesicherung. Durch Selektionierung von einzelnen Patientensubgruppen, mithilfe des in der Datenbank integrierten AuswahlabfrageGenerators, korrelierten wir unter anderem das Alter und den initialen KarnofskyIndex mit einer Survivalanalyse. Es zeigte sich erwartungsgemäß, dass insbesondere das Alter, aber auch der Karnofsky-Index zu Beginn der Erkrankung, ungeachtet der therapeutischen Maßnahmen, großen Einfluss auf die Überlebenswahrscheinlichkeit hatte. Die Operation als primäre therapeutische Maßnahme diente, neben der definitiven histologischen Diagnosesicherung, auch der weitestgehenden Tumorentfernung. Die prognostische Bedeutung der makroskopischen Vollresektion wird in der Literatur kontrovers diskutiert. Wir stellten fest, dass bei gleicher adjuvanter Therapie die Patienten mit makroskopischer Vollresektion eine deutlich bessere Überlebenswahrscheinlichkeit hatten als jene mit einer Resttumorlast. Allerdings war die Bedeutung der adjuvanten Therapien der Bedeutung des Resektionsausmaßes in Bezug auf die Überebenswahrscheinlichkeit statistisch überlegen. Die Strahlentherapie, die seit 25 Jahren unangezweifelt zur Standardtherapie des Glioblastoms gehört, hatte auch in unserer Untersuchung eine klare Verbesserung der medianen Überlebenszeit und auch der progressfreien Überlebenszeit ergeben. Die zusätzliche Behandlung mit Zytostatika erbrachte für die Patienten eine weitere Verbesserung der medianen Überlebenszeit und des progressfreien Intervalls, verglichen mit der alleinigen Strahlentherapie. Es zeigte sich jedoch, dass sich die Chemotherapien untereinander (ACNU als first-line Chemotherapie mit Wechsel auf Temozolomid als second-line Chemotherapie , Temozolomid als Monotherapie und konkomitant) durchaus vergleichbar bezüglich des medianen Gesamtüberlebens waren und hinsichtlich des bildmorphologisch-progressfreien Überlebens von ca 6 Monaten als gleichwertig zu betrachten waren. Die ACNU Monotherapie wies auffallend schlechtere Ergebnisse auf. Bei dieser Therapie betrug das klinische und bilmorphologisch-progressfreie Intervall 4 Monate, die mediane Überlebenszeit 9 Monate. Die anderen adjuvanten Chemotherapien zeigten mediane Überlebenszeiten zwischen 16 und 19 Monaten. Dabei war die konkomitante Temozolomidtherapie mit anschließender Temozolomidmonotherapie, mit einer medianen Überlebenszeit von 19 Monaten und einer 1- 
Jahres-Überlebenswahrscheinlichkeit von $75 \%$, führend. Ob die erstmals im Jahr 2004 in Göttingen eingeführte und mittlerweile als Standardtherapie angewendete konkomitante Chemotherapie mit Temozolomid ein längeres Überleben gewährleistet als die alleinige Temozolomidtherapie im Anschluss an die Bestrahlung, kann aufgrund der vergleichsweise geringen Patientenzahl noch nicht mit Sicherheit festgestellt werden. Offensichtlich zeichnet sich hier jedoch eine deutlich positive Tendenz ab. Die Fortsetzung der Datenanalysen ab dem Jahr 2004 wird hier im Vergleich mit den bisherigen Ergebnissen aussagekräftige Ergebnisse liefern, um die in der aktuellen Literatur beschriebene Überlegenheit von Temozolomid konkomitant auch gegenüber anderen alkylierenden Zytostatika an einem nicht-selektionierten Patientenkollektiv zu bestätigen. Neuere Untersuchungen auf molekularer Ebene lassen vermuten, dass bei den zukünftigen Therapieansätzen das Eingreifen auf genetischer Ebene immer weiter im Vordergrund stehen wird. Solange diese Therapien jedoch weitestgehend experimentellen Charakter haben, sind Studien, wie die hier vorliegende, ein essentieller Bestandteil der Qualitätskontrolle für die behandelnden Disziplinen. Vergleichen wir die Therapieergebnisse von 1998 (1-JahresÜberlebenswahrscheinlichkeit bei 30\%) mit dem Jahr 2004 (1-JahresÜberlebenswahrscheinlichkeit 59\%), zeigt sich hier auf den ersten Blick eine erhebliche prognostische Verbesserung. Diese Ergebnisse sind jedoch stets unter Berücksichtigung der unterschiedlichen Zusammensetzung hinsichtlch prognostischer und therapeutischer Einflussfaktoren in den Patientengruppen der einzelnen Jahre zu werten. Es handelt sich bei unserer Untersuchung im Unterschied zu den bekannten prospektiven Studien um ein inhomogenes Patientenkollektiv aus dem klinischen Altag. Durch Aufschlüsselung der einzelnen Patientensubgruppen, nach Einflussfaktoren auf das Therapieergebnis, werden diese in unserer Untersuchung jedoch transparent dargestellt, und mit steigender Anzahl von Datensätzen lassen sich zunehmend homogene Patientengruppen aus der Datenbank selektieren, die prospektiven Studien in ihrer Aussagekraft sehr nahe kommen.

Nach umfangreicher Analyse einer Vielzahl von klinischen Verläufen von Patienten mit Glioblastom aus der Universitätsklinik in Göttingen zeigt sich, dass eine weitestgehende Tumorresektion sowie radiotherapeutische und chemotherapeutische adjuvante Therapiemaßnahmen das Gesamtüberleben und das klinisch progressfreie Überleben deutlich verlängern. Die Zeit bis zum bildmorphologischen Tumorprogress wird hingegen wenig beeinflusst. Die Patienten lebten somit vergleichsweise länger, jedoch mit einer Rest- oder Rezidivtumorlast. Eine intensive Weiterent- 
wicklung und Qualitätskontrolle auf chirurgischer und adjuvanter Therapieebene ist dringend erforderlich und wird unterstützt durch eine strukturierte Erfassung der Therapieergebnisse einzelner neuroonkologischer Zentren. 


\section{Literaturverzeichnis}

Armitage JO, Carbone PP, Connors JM, Levine A, Benett JM, Kroll S (2003): Treatmentrelated myelodyplasia and acute leukaemia in non- Hodgkin $\mathrm{s}$ lymphoma patients.

J Clin Oncol 10, 3728-36

Barker FG 2nd, Davis RL, Chang SM, Prados MD (1996): Necrosis as a prognostic factor in glioblastoma multiforme

Cancer $\underline{77}(6), 1161-66$.

Behin A, Hoang-Xuan K, Carpentier AF, Delattre JY (2003):Primary brain tumors in adults

The Lancet $\underline{361,323-31}$

Brock CS, Newlands ES, Wedge SR, Bower M, Evans H, Colquhoun I, Roddie M, Glaser M, Brampton MH, Rustin GJ (1998): Phase I trial of temozolomide using an extended continuous oral schedule.

Cancer Res $\underline{58}$, 4363-67

Burger PC, Pearl DK, Aldape K, Yates AJ, Scheithauer BW, Passe SM, Jenkins RB, James CD (2001): Small cell architecture--a histological equivalent of EGFR amplification in glioblastoma multiforme?

J Neuropathol Exp Neurol. 60(11), 1099-104. 
Cairncross JG, Ueki K, Zlatescu MC, Lisle DK, Finkelstein DM, Hammond RR, Silver JS, Stark PC, Macdonald DR, Ino Y, Ramsay DA, Louis DN (1998): Specific genetic predictors of chemotherapeutic response and survival in patients with anaplastic Oligodendrogliomas. J Natl Cancer Inst $\underline{90}$, 1473-79

Chang $\mathrm{CH}$, Horton J, Schoenfeld D et al. (1983): Comparison of postoperative radiotherapy and combined postoperative radiotherapy and chemotherapy in the multidisciplinary management ofmalignant gliomas:a joint Radiation Therapy Oncology Group study.

Cancer $\underline{52}, 997-1007$

Dropcho EJ, Soong SJ (1996): The prognostic impact of prior low grade histology in patients with anaplastic gliomas: a case control study Neurology $\underline{47}(3), 684-90$

Emami B, Lyman J, Brown A et al. (1991): Tolerance of normal Tissue of therapeutic irradiation.

Int J Radiat Oncol Biol Phys 21,109-22

Galanis E, Buckner JC, Dinapoli RP, Scheithauer BW, Jenkins RB, Wang CH, O FalIon JR, Farr G (1998): Clinical outcome of gliosarcoma compared with glioblastoma multiforme

J Neurosurg $\underline{89}, 425-430$

Giese A, Westphal M (1996): Glioma invasion in the central nervous system Neurosurgery $\underline{39}(2), 235-50$ 
Gil-Benso R, Lopez-Gines C, Benito R, López-Guerrero JA, Callaghan RC, Pellín A, Roldán P, Cerdá-Nicolas M (2007): Concurrent EGFR amplification and TP-53 mutation in glioblastomas.

Clin Neuropathol 26 $(5), 224-31$

GMT Group (Glioma Meta-analysis Trialists) (2002): Chemotherapy in adult highgrade glioma: a systematic review and meta-analysis of individual patient data from 12 randomized trials.

Lancet $\underline{359}, 1011-18$

Green SB, Byar DP, Walker MD, Pistenmaa DA, Alexander E Jr, Batzdorf U, Brooks WH, Hunt WE, Mealey J Jr, Odom GL, Paoletti P, Ransohoff J 2nd, Robertson JT, Selker RG, Shapiro WR, Smith KR Jr, Wilson CB, Strike TA (1983): Comparisons of carmustine, procarbazine, and high-dose methylprednisolone as additions to surgery and radiotherapy for the treatment of malignant glioma.

Cancer Treat Rep 67(2), 121-32.

Gross MW, Weber WA, Feldmann HJ, Bartenstein P, Schwaiger M, Molls M. (1998): The value of F-18-flourodeoxyglucose PETforthe 3-D radiation treatment planning of malignant gliomas.

Int J Radiat Oncol Biol Phys 411(5), 989-95

Hart MG, Grant R, Metcalfe SE (2000): Biopsy versus resection for high grade glioma. Cochrane

Database of Systematic Reviews 2. Art. No.: CD002034. DOI:

He J, Mokhtari K, Sanson M (2001): Glioblastoma with an oligodendroglial component: a pathological and molecular study.

J Neuropathol Exp Neurol $\underline{60}$, 863-71 
Hegi ME, Diserens AC, Gorlia T, Hamou MF, de Tribolet N, Weller M, Kros JM, Hainfeller JA, Mason W, Mariani Luigi (2005): MGMT Gene Silencing and Benefit from Temozolomide in Glioblastoma

N Engl J Med 352, 997-1003

Hess CF, Schaaf JC, Kortmann RD et al. (1994): Malignant glioma: patterns of failure following individually tailored limited volume irradiation.

Radiother Oncol $\underline{30}$, 146-49

Hilton DA, Penney M, Pobereskin L, Sanders H, Love S (2004): Histological indicators of prognosis in glioblastomas: retinoblastoma protein expression and oligodendroglial differentiation indicate improved survival.

Histopathology $\underline{44}(6), 555-6$

Hofer S, Merlob A (2002): Therapeutische Optionen für maligne Gliome WHO-Grad III und IV.

Schweiz Med Forum $\underline{32 / 33}, 748-55$

Ichimura K, Ohgaki H, Kleihues P, Collins VP (2004): Molecular pathogenesis of astrocytic tumours.

J Neurooncol $\underline{70}(2), 137-60$

James CD, Burger PC, Pearl DK, Aldape K, Yates AJ, Scheithauer BW, Passe SM, Jenkins RB (2001): Small cell architecture-a histological equivalent of EGFR, amplification in glioblastoma multiforme?

J Neuropathol Exp Neurol $\underline{60}(11), 1099-104$ 
Jeremic B, Grujicic D, Antunovic V, Djuric L, Stojanovic M, Shibamoto Y (1994): Influence of extent of surgery and tumor location on treatment outcome of patients with glioblastoma multiforme treated with combined modality approach.

J Neurooncol 21, 177-85

Kleihues P, Ohgaki H (1999): Primary and secondary glioblastoma: from a concept to clinical diagnosis.

Neuro Oncology $\underline{1}, 44-51$

Kleihues P, Burger PC, Scheithauer BW (1993): The new WHO classification of brain tumours

Brain Pathol $\underline{3}, 255-68$

Kleihues P, Louis DN, Scheithauer BW, Rorke LB, Reifenberger G, Burger PC, Cavenee WK (2002): The WHO classification of tumors of the nervous system J Neuropathol Exp Neurol 61(3), 215-25, discussion 226-9.

Kostron H, Roessler K (2006): Surgical intervention in Patients with malignant Glioma.

Wien Med Wochenschr 156(11-12), 338-41

Lacroix M, Abi-Said D, Fourney DR, et al. (2001): A multivariate analysis of 416 patients with glioblastoma multiforme: prognosis, extent of resection, and survival. J Neurosurg $\underline{95}, 190-98$

Laperriere N, Zuraw L, Cairncross G (2002): Radiotherapy for newly diagnosed malignant glioma in adults: a systematic review.

Radiother Oncol 63(3), 259-73 
Legler JM, Gloeckler Ries LA, Smith MA, Warren JL, Heinemann EF, Kaplan RS, Linet MS (1999): Response: Brain and Other Central Nervous System Cancers: Recent Trends in Incidence and Mortality.

J Natl Cancer Inst 91(23), 2050A-22051

Levin VA, Silver P, Hannigan J, Wara WM, Gutin PH, Davis RL, Wilson CB (1990): Superiority of post-radiotherapy adjuvant chemotherapy with CCNU, procarbazine, and vincristine (PCV) over BCNU for anaplastic gliomas: NCOG final report. Int J Radiat Oncol Biol Phys 18(2), 321-24

Medical Research Council Brain Tumor Working Party (2001): Randomized trial of procarbazine, lomustine and vincristine in the adjuvant treatment of high-grade astrocytoma: A Medical Research Council trial.

J Clin Oncol $\underline{19}, 509-18$

Nitta T, Sato K (1995): Prognosic implication of the extent of surgical resection in patients with intracranial malignant gliomas.

Cancer $\underline{75}$, 2727-31

Prados M, Scott C, Curran W, Nelson D, Leibel S, Kramer S (1999): Procarbazine, Lomustine and Vincristine (PCV) chemotherapy for anaplastic astrozytoma: A retrospective review of Radiation Therapy Oncology Group protocols comparing survival with Carmustine or PCV adjuvant chemotherapy

J Clin Oncol $\underline{44}$, 3389-95

Salazar OM, Rubin P, Feldstein ML et al. (1979): High dose radiation therapy in the treatment of malignant gliomas: final report.

Int J Radiat Oncol Biol Phys $\underline{5}$, 1733-40 
Shapiro WR, Green SB, Burger PC et al (1989): Randomized trial of three chemotherapies regimes and two radiotherapy regimens in postoperative treatment of malignant glioma: Brain Tumor Cooperative Group trial 8001

J Neurosurg $\underline{71}, 1-9$

Shapiro WR (1992): Chemotherapie of malignant Gliomas: Studies of the BTCG Rev. Neurol. $\underline{148}, 428-34$

Stewart LA (2002): Chemotherapie in adult high-grade glioma: a systematic review and metaanalysis of individual patient data from 12 randomized trials.

Lancet $\underline{359}$ 1011-18

Stummer W, Reulen HJ, Nowotny A, et al (2003): Fluorescence-guided resection of malignant gliomas. An overview

Acta Neurochir $\underline{88}, 9-12$

Stupp R, Warren P, Mason WP, van den Bent M, Weller M, Fisher B, Tapophoorn MJB, Belanger K, Brandes AA, Marosi C (2005): Radiotherapy plus Concomitant and Adjuvant Temozolomide for Glioblastoma

N Engl J Med 352, 987-96

Van den Bent MJ, Chinot O, Boogerd W, Bravo Marques J, Taphoorn MJB, Kros JM, van der Rijt CCD, Vecht CJ, De Beule N, Baron B (2003): Second-line chemotherapie with temozolomide in recurrent oligodendroglioma after PCV chemotherapie: EORTC Brain Tumor Group phase II study 26972

Ann Oncol 14(4), 599-602 
Walker MD, Alexander E Jr, Hunt We MacCarty CS, Mealey MS Jr, Norell HA, Owens G, Ronsohoff J, Wilson CB, Gehan EA, Strike TA ( 1978): Evaluation of $\mathrm{BCNU}$ and/or radiotherapy in the treatment of anaplasic gliomas. A cooperative clinical trial.

J Neurosurg $\underline{49}(3), 333-43$

Walker MD, Strike TA, Sheline GE et al. (1979): An analysis of dose-effect relationship of malignant gliomas.

Int J Radiat Oncol Biol Phys $\underline{5}, 1725-3$

Watanabe K, Tachibana O, Sata K, Yonekawa Y, Kleihues P, Ohgaki H (1996): Overexpression of the EGF receptor and p 53 mutations are mutually exclusive in the evolution of primary and secondary glioblastomas

Brain pathol $\underline{6}, 217-23$

Weber W, Bartenstein P, Gross MW, Kinzel D, Daschner H, Feldmann HJ, Reidel G, Ziegler SI, Lumenta C, Molls M, Schwaiger M.(1997): Fluorine-18-FDG PET and londine-123-IMT SPECT in the evaluation of brain tumor J Nucl Med $\underline{38}(5), 802-8$

Weller M, Schmidt C, Roth W, Dichgans J (1997): Chemotherapy of human malignant glioma: prevention of efficacy by dexamethasone?

Neurology $\underline{48}, 1704-09$

Weller M, von Deimling A, Ernemann U, Schackert G, Bamberg M (2003): Oligodendriome (2003)

Der Onkologe $\underline{9}, 729-38$ 
Weller M, Tonn JC, Ernemann U, Wiestler OD, Bamberg M (2006): Diagnostik und Therapie des Glioblastoms

Der Onkologe 12, 500-07

Werner-Wasik M, Scott CB, Nelson DF, Gaspar LE, Murray KJ, Fischbach JA, Nelson JS, Weinstein AS, Curran WJ Jr (1996): Final report of a phase I/II trial of hyperfractionated and accelerated hyperfractionated radiation therapy with carmustine for adults with supratentorial malignant gliomas. Radiation Therapy Oncology Group Study 83-02.

Cancer $\underline{77}(8), 1535-43$

Zhou XP, Li YJ, Hoang-Xuan K, et al (1999): Mutational analysis of the PTEN gene in gliomas: molecular and pathologcal correlations.

Int J Cancer $\underline{83}, 150-54$ 